\title{
COMPACTNESS CRITERION FOR SEMIMARTINGALE LAWS AND SEMIMARTINGALE OPTIMAL TRANSPORT
}

\author{
CHONG LIU AND ARIEL NEUFELD
}

\begin{abstract}
We provide a compactness criterion for the set of laws $\mathfrak{P}_{\text {sem }}^{a c}(\Theta)$ on the Skorokhod space for which the canonical process $X$ is a semimartingale having absolutely continuous characteristics with differential characteristics taking values in some given set $\Theta$ of Lévy triplets. Whereas boundedness of $\Theta$ implies tightness of $\mathfrak{P}_{\text {sem }}^{a c}(\Theta)$, closedness fails in general, even when choosing $\Theta$ to be additionally closed and convex, as a sequence of purely discontinuous martingales may converge to a diffusion. To that end, we provide a necessary and sufficient condition that prevents the purely discontinuous martingale part in the canonical representation of $X$ to create a diffusion part in the limit. As a result, we obtain a sufficient criterion for $\mathfrak{P}_{\text {sem }}^{a c}(\Theta)$ to be compact, which turns out to be also a necessary one if the geometry of $\Theta$ is similar to a box on the product space.

As an application, we consider a semimartingale optimal transport problem, where the transport plans are elements of $\mathfrak{P}_{\text {sem }}^{a c}(\Theta)$. We prove the existence of an optimal transport law $\widehat{\mathbb{P}}$ and obtain a duality result extending the classical Kantorovich duality to this setup.
\end{abstract}

\section{INTRODUCTION}

The goal of this paper is to provide a compactness criterion for semimartingale laws. Given a set $\Theta$ of Lévy triplets, we denote by $\mathfrak{P}_{\text {sem }}^{a c}(\Theta)$ the set of all probability measures $\mathbb{P}$ on the Skorokhod space for which the canonical process $X=\left(X_{t}\right)_{0 \leq t \leq T}$ is a semimartingale with differential characteristics taking values in $\Theta$; this means that the semimartingale characteristics $\left(B^{\mathbb{P}}, C^{\mathbb{P}}, \nu^{\mathbb{P}}\right)$ are of the form $\left(b_{t}^{\mathbb{P}} d t, c_{t}^{\mathbb{P}} d t, F_{t}^{\mathbb{P}} d t\right)$, and the processes $\left(b^{\mathbb{P}}, c^{\mathbb{P}}, F^{\mathbb{P}}\right)$ evolve in $\Theta$. For simplicity in the introduction, let us only consider laws with starting point $X_{0}=0$. We restrict our attention to sets of Lévy triplets $\Theta \subseteq \mathbb{R}^{d} \times \mathbb{S}_{+}^{d} \times \mathcal{L}$ satisfying the boundedness condition

$$
\sup _{(b, c, F) \in \Theta}\left\{|b|+|c|+\int_{\mathbb{R}^{d}}|x|^{2} \wedge|x| F(d x)\right\}<\infty,
$$

where $\mathcal{L}$ denotes the set of Lévy measures. It turns out that the condition (1) on $\Theta$ implies tightness of $\mathfrak{P}_{\text {sem }}^{a c}(\Theta)$ and that under any limit law $\mathbb{P}_{0}$ of a sequence $\left(\mathbb{P}_{n}\right) \subseteq \mathfrak{P}_{\text {sem }}^{a c}(\Theta)$ the canonical process remains a semimartingale with absolutely continuous characteristics; see Corollary 3.22 and Proposition 4.4 .

Finding tightness conditions on semimartingale laws is a well-studied problem. In [26], tightness of solutions of a martingale problem is proven. In fact, (1) can be

Received by the editors April 30, 2017, and, in revised form, February 9, 2018.

2010 Mathematics Subject Classification. Primary 60F05, 60G44, 93E20.

Financial support by the NAP Grant and the Swiss National Foundation Grant SNF 200021_153555 is gratefully acknowledged. 
seen as the analogue of the boundedness conditions on the triplet in the integrodifferential operator of the martingale problem in 26]. In [35, conditions on the predictable quadratic covaration of a sequence of locally square-integrable martingales $\left(M_{n}\right)$ is studied, which guarantee tightness of the laws of $\left(M_{n}\right)$. Tightness for semimartingale laws was studied in 27] on the space of càdlàg paths but endowed with a weaker topology than the usual Skorokhod $J_{1}$-topology, which makes it easier to obtain tightness. They show that a sequence of quasimartingales laws with uniformly bounded conditional variation is tight, and any such limit is a quasimartingale law. We refer to [6, 20,21 for general tightness results for processes.

For continuous semimartingales, i.e., when $F \equiv 0$, it was shown in 39] using techniques developed in [41] that if in addition to (1) $\Theta \subseteq \mathbb{R}^{d} \times \mathbb{S}_{+}^{d}$ is closed and convex, then $\mathfrak{P}_{\text {sem }}^{a c}(\Theta)$ is compact. In other words, in the continuous case, closedness and boundedness of $\Theta$ provide compactness of the corresponding set of probability measures $\mathfrak{P}_{\text {sem }}^{a c}(\Theta)$.

However, on the Skorokhod space, it is straightforward to see that closedness of $\mathfrak{P}_{\text {sem }}^{a c}(\Theta)$ may fail, even when choosing $\Theta$ in addition to (1), to be closed (and convex). We are not aware of any closedness criterion of that type. To understand the difficulty, consider in dimension one the set

$$
\Theta:=\{(b, c, F) \in \mathbb{R} \times[0, \infty) \times \mathcal{L} \mid b=0, c=0, \operatorname{supp}(F) \subseteq\{|x| \leq 1\}\} .
$$

We see that $\Theta$ satisfies (1), is convex, and it is straightforward to argue that $\Theta$ is closed. However, $\Theta$ contains the sequence of Lévy triplets $\left(\left(0,0, n \delta_{\frac{1}{\sqrt{n}}}\right)\right)_{n \in \mathbb{N}}$. Consider the sequence of laws $\left(\mathbb{P}_{n}\right) \subseteq \mathfrak{P}_{\text {sem }}^{a c}(\Theta)$, where each element $\mathbb{P}_{n}$ is defined as the unique law such that $X$ becomes a Lévy process with triplet $\left(0,0, n \delta_{\frac{1}{\sqrt{n}}}\right)$. Under each $\mathbb{P}_{n}, X$ is the scaled compensated Poisson process $\frac{1}{\sqrt{n}}\left(N_{t}^{n}-n t\right)$ with intensity $n$, which is known to converge weakly to Brownian motion. However, the Wiener measure $\mathbb{P}_{0}$ is not an element of $\mathfrak{P}_{\text {sem }}^{a c}(\Theta)$, as the corresponding triplet $(0,1,0)$ is not an element of $\Theta$. So closedness of $\mathfrak{P}_{\text {sem }}^{a c}(\Theta)$ fails.

The key difficulty of getting closedness of $\mathfrak{P}_{\text {sem }}^{a c}(\Theta)$ is that a sequence of purely discontinuous martingales may converge to a diffusion, and this (additional) diffusion may destroy the closedness of $\mathfrak{P}_{\text {sem }}^{a c}(\Theta)$, even when choosing $\Theta$ to be closed. Therefore, we need to characterize the condition on $\Theta$ that prevents the purely discontinuous (local) martingale part of $X$ to create an additional diffusion part in the limit.

We show in Theorem 2.1 that any limit of a sequence $\left(\mathbb{P}_{n}\right)$ of purely discontinuous martingale laws being in $\mathfrak{P}_{\text {sem }}^{a c}(\Theta)$ is a martingale law. The necessary and sufficient condition for the limit $\mathbb{P}_{0}$ to be a purely discontinuous martingale law turns out to be

$$
\lim _{\delta \downarrow 0} \limsup _{n \rightarrow \infty} \mathbb{E}^{\mathbb{P}_{n}}\left[\int_{0}^{T} \int_{\{|x| \leq \delta\}}|x|^{2} F_{t}^{\mathbb{P}_{n}}(d x) d t\right]=0 .
$$

Now, the intuition is the following. If we can exclude the sequence of purely discontinuous martingale parts to create an additional diffusion in the limit, then the boundedness condition (1) together with closedness of $\Theta$ should lead to compactness of $\mathfrak{P}_{\text {sem }}^{a c}(\Theta)$. The condition

$$
\lim _{\delta \downarrow 0} \sup _{(b, c, F) \in \Theta} \int_{\{|x| \leq \delta\}}|x|^{2} F(d x)=0
$$


is the weakest condition on $\Theta$ one can impose that prevents limits of purely discontinuous martingales to possess a diffusion part.

We show in Theorem 2.5 that if $\Theta$ is closed, convex, and satisfies (1), then (2) is a sufficient condition that $\mathfrak{P}_{\text {sem }}^{a c}(\Theta)$ is compact. Moreover, if the geometry of $\Theta \subseteq \mathbb{R}^{d} \times \mathbb{S}_{+}^{d} \times \mathcal{L}$ is similar to the one of a box on the product space (see Definition 2.4), then (2) is also a necessary condition.

As an application of the compactness criterion for $\mathfrak{P}_{\text {sem }}^{a c}(\Theta)$, we study a semimartingale optimal transport problem. In classical optimal transport theory, the Monge-Kantorovich transport problem for given distributions $\mu_{0}, \mu_{1}$ on $\mathbb{R}^{d}$ and cost function $c: \mathbb{R}^{d} \times \mathbb{R}^{d} \rightarrow[0, \infty)$ consists of minimizing the functional

$$
\mathbb{P} \mapsto \mathbb{E}^{\mathbb{P}}\left[c\left(X_{0}, X_{1}\right)\right]
$$

over all distributions $\mathbb{P}$ on $\mathbb{R}^{d} \times \mathbb{R}^{d}$ with initial marginal $\mathbb{P} \circ X_{0}^{-1}=\mu_{0}$ and final marginal $\mathbb{P} \circ X_{1}^{-1}=\mu_{1}$. A duality result was established in [24, 25] under some suitable conditions on the cost function $c$. We refer to [40] as a reference for classical optimal transport. Recently, martingale optimal transport has been a very active field of research $([1,3,4,8,14,15,17)$, also in connection to Skorokohod Embedding Problems $([2,16,18,19,34)$ and to robust pricing in mathematical finance $([1,3$, 11, 14, 37), to name but a few.

Semimartingale optimal transport was introduced in 30 as an extension of the classical Monge-Kantorovich optimal transport problem, where for a given cost function $c^{\mathbb{P}}(X)$ (which may vary in $\mathbb{P}$ ) and given distributions $\mu_{0}, \mu_{1}$ on $\mathbb{R}^{d}$, one minimizes

$$
\mathbb{P} \mapsto \mathbb{E}^{\mathbb{P}}\left[c^{\mathbb{P}}(X)\right]
$$

over all semimartingale laws $\mathbb{P}$ with initial marginal $\mathbb{P}_{\circ} X_{0}^{-1}=\mu_{0}$ and final marginal $\mathbb{P} \circ X_{1}^{-1}=\mu_{1}$. We see that the semimartingale optimal transport problem recovers the classical Monge-Kantorovich optimal transport problem when choosing $c^{\mathbb{P}}(X) \equiv$ $c\left(X_{0}, X_{1}\right)$.

We study the following semimartingale optimal transport problem. For any distributions $\mu_{0}, \mu_{1}$ on $\mathbb{R}^{d}$ we denote by $\mathfrak{P}_{\Theta}\left(\mu_{0}, \mu_{1}\right)$ the set of all probability measures $\mathbb{P} \in \mathfrak{P}_{\text {sem }}^{a c}(\Theta)$ which have initial marginal $\mathbb{P} \circ X_{0}^{-1}=\mu_{0}$ and final marginal $\mathbb{P} \circ X_{1}^{-1}=\mu_{1}$. Then, given a cost function $L$, distributions $\mu_{0}, \mu_{1}$ on $\mathbb{R}^{d}$, and a set $\Theta \subseteq \mathbb{R}^{d} \times \mathbb{S}_{+}^{d} \times \mathcal{L}$, we want to minimize the functional

$$
\mathbb{P} \mapsto \mathbb{E}^{\mathbb{P}}\left[\int_{0}^{1} L\left(t, X, b_{t}^{\mathbb{P}}, c_{t}^{\mathbb{P}}, F_{t}^{\mathbb{P}}\right) d t\right]
$$

over all probability measures $\mathbb{P} \in \mathfrak{P}_{\Theta}\left(\mu_{0}, \mu_{1}\right)$. We prove the existence of a minimizer $\widehat{\mathbb{P}} \in \mathfrak{P}_{\Theta}\left(\mu_{0}, \mu_{1}\right)$ and a duality theorem which is an extension of the classical Kantorovich duality to this setup (see [24,25]). When choosing $\Theta:=\mathbb{R}^{d} \times\left\{I_{d \times d}\right\} \times\{0\}$ and $\Theta \subseteq \mathbb{R}^{d} \times \mathbb{S}_{+}^{d} \times\{0\}$, we recover the semimartingale optimal transport and the corresponding strong duality result in [30] and 39, respectively. We also refer to [9, 29] for related work on semimartingale optimal transport for continuous semimartingales. Following the arguments of [30,39], the existence of a minimizer $\widehat{\mathbb{P}}$ and the duality theorem is obtained in Theorem 2.16 using classical convex duality results, which require that the value function

$$
\left(\mu_{0}, \mu_{1}\right) \mapsto V\left(\mu_{0}, \mu_{1}\right):=\inf _{\mathbb{P} \in \mathfrak{P}_{\Theta}\left(\mu_{0}, \mu_{1}\right)} E^{\mathbb{P}}\left[\int_{0}^{1} L\left(t, X, b_{t}^{\mathbb{P}}, c_{t}^{\mathbb{P}}, F_{t}^{\mathbb{P}}\right) d t\right]
$$


be convex and lower semicontinuous. The lower semicontinuity of the value function heavily depends on the compactness property $\mathfrak{P}_{\text {sem }}^{a c}(\Theta)$, which we now can ensure from the compactness criterion by imposing $\Theta$ to satisfy (11) and (2).

The remainder of this paper is organized as follows. In Section 2, we introduce the notions and present the main results. In Section 3, we lift the laws $\mathfrak{P}_{\text {sem }}^{a c}(\Theta)$ to an enlarged space $\bar{\Omega}$, where we prove our main results about the characterization when purely discontinuous martingales can create a diffusion in the limit and the compactness criterion for the semimartingale laws. The enlarged space allows us to consider the joint distribution of the summands in the canonical representation of $X$. The main tool for the proof is the Skorokhod representation theorem, which enables us to translate weak convergence to pointwise convergence. In Section 4, we prove the above results on the original Skorokhod space using the corresponding results derived on the enlarged space. To that end, an analysis of semimartingale characteristics with respect to a filtration smaller than the original one on the enlarged space is needed. In Section 5, we prove the existence of a minimizer and the duality result of the semimartingale optimal transport problem.

\section{SETUP AND MAIN RESUltS}

Fix $d \in \mathbb{N}, T<\infty$, and let $\Omega:=\mathbb{D}\left([0, T], \mathbb{R}^{d}\right)$ be the space of all càdlàg paths $\omega=$ $\left(\omega_{t}\right)_{0 \leq t \leq T}$ endowed with the usual Skorokhod $J_{1}$-topology, where we equip $\Omega$ with the corresponding Borel $\sigma$-field $\mathcal{F}$. Denote by $X=\left(X_{t}\right)_{0 \leq t \leq T}$ the canonical process $X_{t}(\omega)=\omega(t)$ and by $\mathbb{F}=\left(\mathcal{F}_{t}\right)_{0 \leq t \leq T}$ the (raw) filtration generated by $X$. Moreover, we denote by $\mathfrak{M}_{1}(\Omega)$ the space of all probability measures on $(\Omega, \mathcal{F})$, which is a Polish space by the usual topology of weak convergence; see e.g. [5, Chapter 7].

For a given probability measure $\mathbb{P}$ and a filtration $\mathbb{G}$ on $(\Omega, \mathcal{F})$, we call a $\mathbb{G}$ adapted process $Y$ with càdlàg paths a $\mathbb{P}-\mathbb{G}$-semimartingale if there exist rightcontinuous, $\mathbb{G}$-adapted processes $M$ and $A$ with $M_{0}=A_{0}=0$ such that $M$ is a $\mathbb{P}$ - $\mathbb{G}$-local martingale and $A$ has paths of finite variation $\mathbb{P}$-a.s. such that $Y=$ $Y_{0}+M+A \mathbb{P}$-a.s.

Fix a continuous truncation function $h: \mathbb{R}^{d} \rightarrow \mathbb{R}^{d}$, that is, a bounded continuous function such that $h(x)=x$ in a neighborhood of zero. Let $\mathbb{P}$ be a probability measure on $(\Omega, \mathcal{F})$ under which the canonical process $X$ is a $\mathbb{P}-\mathbb{F}$-semimartingale. Denote by $\left(B^{\mathbb{P}}, C^{\mathbb{P}}, \nu^{\mathbb{P}}\right)$ its $\mathbb{P}-\mathbb{F}$-semimartingale characteristics. This means that $\left(B^{\mathbb{P}}, C^{\mathbb{P}}, \nu^{\mathbb{P}}\right)$ is a triplet of processes such that $\mathbb{P}$-a.s., $B^{\mathbb{P}}$ is the predictable finite variation part in the canonical decomposition of

$$
X-\sum_{0 \leq s \leq}\left(\Delta X_{s}-h\left(\Delta X_{s}\right)\right)
$$

under $\mathbb{P}, C^{\mathbb{P}}$ is the quadratic covariation of the continuous local martingale part of $X$ under $\mathbb{P}$, and $\nu^{\mathbb{P}}$ is the $\mathbb{P}$-compensator of the measure $\mu^{X}$ associated to the jumps of $X$. We refer to [21] as our main reference for standard notions related to general semimartingale theory. Under a given probability measure $\mathbb{P}, X$ is a $\mathbb{P}$ - $\mathbb{F}$-semimartingale if and only if it is one with respect to the right continuous filtration $\mathbb{F}_{+}$or the usual augmentation $\mathbb{F}_{+}^{\mathbb{P}}$, and the semimartingale characteristics with these filtrations are the same; see [31, Proposition 2.2]. We denote by $\mathfrak{P}_{\text {sem }}$ the set of all probability measures on $(\Omega, \mathcal{F})$ such that $X$ is a $\mathbb{P}-\mathbb{F}$-semimartingale. 
Given a semimartingale law $\mathbb{P}$, we say that $X$ has absolutely continuous characteristics under $\mathbb{P}-\mathbb{F}$ if $\left(d B^{\mathbb{P}}, d C^{\mathbb{P}}, d \nu^{\mathbb{P}}\right)=\left(b_{t}^{\mathbb{P}} d t, c_{t}^{\mathbb{P}} d t, F_{t}^{\mathbb{P}} d t\right)$. The differential characteristics $\left(b^{\mathbb{P}}, c^{\mathbb{P}}, F^{\mathbb{P}}\right)$ take values in $\mathbb{R}^{d} \times \mathbb{S}_{+}^{d} \times \mathcal{L}$, where $\mathbb{S}_{+}^{d}$ denotes the set of all symmetric, nonnegative definite $d \times d$ matrices and

$$
\mathcal{L}:=\left\{F \text { measure on }\left.\mathbb{R}^{d}\left|\int_{\mathbb{R}^{d}}\right| x\right|^{2} \wedge 1 F(d x)<\infty \text { and } F(\{0\})=0\right\}
$$

denotes the set of Lévy measures. We write

$$
\mathfrak{P}_{\text {sem }}^{a c}:=\left\{\mathbb{P} \in \mathfrak{P}_{\text {sem }} \mid X \text { has absolutely continuous characteristics }\right\} \text {. }
$$

Given a set $\Theta \subseteq \mathbb{R}^{d} \times \mathbb{S}_{+}^{d} \times \mathcal{L}$, we denote by

$$
\mathfrak{P}_{\text {sem }}^{a c}(\Theta):=\left\{\mathbb{P} \in \mathfrak{P}_{\text {sem }}^{a c} \mid\left(b^{\mathbb{P}}, c^{\mathbb{P}}, F^{\mathbb{P}}\right) \in \Theta \mathbb{P} \times d t \text {-a.e. }\right\}
$$

the set of all semimartingale laws in $\mathfrak{P}_{\text {sem }}^{a c}$ with differential characteristics taking value in $\Theta \mathbb{P} \times d t$-a.s. Given a set $\Gamma_{0} \subseteq \mathfrak{M}_{1}\left(\mathbb{R}^{d}\right)$ of distributions on $\mathbb{R}^{d}$, we write

$$
\mathfrak{P}_{\text {sem }}^{a c}(\Theta)\left(\Gamma_{0}\right):=\left\{\mathbb{P} \in \mathfrak{P}_{\text {sem }}^{a c}(\Theta) \mid \mathbb{P} \circ X_{0}^{-1} \in \Gamma_{0}\right\}
$$

for those elements in $\mathfrak{P}_{\text {sem }}^{a c}(\Theta)$ with corresponding initial distribution lying in $\Gamma_{0}$.

To provide our compactness criterion for semimartingale laws, we first need to introduce a topology on $\Theta$. To that end we endow $\mathcal{L}$ with the topology of weak convergence induced by the bounded continuous functions on $\mathbb{R}^{d}$ vanishing in a neighborhood of the origin. More precisely, given $\left(F_{n}\right)_{n \in \mathbb{N}} \subseteq \mathcal{L}$ and $F \in \mathcal{L}$, we say that $\left(F_{n}\right)_{n \in \mathbb{N}}$ converges to $F$ if $\lim _{n \rightarrow \infty} \int_{\mathbb{R}^{d}} g(x) F_{n}(d x)=\int_{\mathbb{R}^{d}} g(x) F(d x)$ for all bounded continuous functions $g$ on $\mathbb{R}^{d}$ vanishing in a neighborhood of the origin. Having defined a topology on $\mathcal{L}$, we can equip $\mathbb{R}^{d} \times \mathbb{S}_{+}^{d} \times \mathcal{L}$ with the corresponding product topology.

Consider the following condition on the values of the differential characteristics.

Condition (B). A set $\Theta \subseteq \mathbb{R}^{d} \times \mathbb{S}_{+}^{d} \times \mathcal{L}$ satisfies Condition (B) if

$$
\mathcal{K}:=\sup _{(b, c, F) \in \Theta}\left\{|b|+|c|+\int_{\mathbb{R}^{d}}|x|^{2} \wedge|x| F(d x)\right\}<\infty .
$$

Note that Condition (B) guarantees that both the continuous and purely discontinuous local martingale parts of the canonical representation of $X$ under any $\mathbb{P} \in \mathfrak{P}_{\text {sem }}^{a c}(\Theta)$ are true martingales, and every element $\mathbb{P} \in \mathfrak{P}_{\text {sem }}^{a c}(\Theta)$ being a $\sigma$ martingale law satisfying $\mathbb{E}^{\mathbb{P}}\left[\left|X_{0}\right|\right]<\infty$ is a true martingale law; see the proof of [32, Lemma 5.2].

Now, let us state our first result, which provides a necessary and sufficient condition for limits of purely discontinuous martingale measures to be again a purely discontinuous martingale measure. To that end, denote

$$
\begin{aligned}
\mathfrak{P}_{m}^{a c} & :=\left\{\mathbb{P} \in \mathfrak{P}_{s e m}^{a c} \mid X \text { is a } \mathbb{P}-\mathbb{F} \text {-martingale }\right\}, \\
\mathfrak{P}_{m, d}^{a c} & :=\left\{\mathbb{P} \in \mathfrak{P}_{m}^{a c} \mid X \text { is a } \mathbb{P}-\mathbb{F} \text {-purely discontinuous martingale }\right\} \\
& =\left\{\mathbb{P} \in \mathfrak{P}_{m}^{a c} \mid C^{\mathbb{P}}=0\right\}, \\
\mathfrak{P}_{m, d}^{a c}(\Theta) & :=\left\{\mathbb{P} \in \mathfrak{P}_{m, d}^{a c} \mid\left(b^{\mathbb{P}}, c^{\mathbb{P}}, F^{\mathbb{P}}\right) \in \Theta \mathbb{P} \times d t \text {-a.e. }\right\} .
\end{aligned}
$$

Theorem 2.1. Let $\Theta \subseteq \mathbb{R}^{d} \times \mathbb{S}_{+}^{d} \times \mathcal{L}$ satisfy Condition (B) and let $\left(\mathbb{P}_{n}\right)_{n \in \mathbb{N}} \subseteq$ $\mathfrak{P}_{m, d}^{a c}(\Theta)$ be a sequence of purely discontinuous martingale measures which converges weakly to some $\mathbb{P}_{0} \in \mathfrak{M}_{1}(\Omega)$ satisfying $\mathbb{E}^{\mathbb{P}_{0}}\left[\left|X_{0}\right|\right]<\infty$. Then the following hold true.

(1) We have $\mathbb{P}_{0} \in \mathfrak{P}_{m}^{a c}$. 
(2) We have the following necessary and sufficient criterion for the limit law to be a purely discontinuous martingale measure:

$$
\mathbb{P}_{0} \in \mathfrak{P}_{m, d}^{a c} \Longleftrightarrow \lim _{\delta \downarrow 0} \limsup _{n \rightarrow \infty} \mathbb{E}^{\mathbb{P}_{n}}\left[\int_{0}^{T} \int_{\{|x| \leq \delta\}}|x|^{2} F_{t}^{\mathbb{P}_{n}}(d x) d t\right]=0 .
$$

Corollary 2.2. Let $\Theta \subseteq \mathbb{R}^{d} \times \mathbb{S}_{+}^{d} \times \mathcal{L}$ satisfy Condition (B) and let $\left(\mathbb{P}_{n}\right)_{n \in \mathbb{N}} \subseteq$ $\mathfrak{P}_{\text {sem }}^{a c}(\Theta)$. Assume that the sequence of laws $\left(\mathbb{P}_{n} \circ\left(M^{d, \mathbb{P}_{n}}\right)^{-1}\right)_{n \in \mathbb{N}}$ of the corresponding purely discontinuous martingale part of the canonical process $X$ under $\mathbb{P}_{n}$ converges to some law $\mathbb{P}_{0} \in \mathfrak{M}_{1}(\Omega)$. Then (1) and (2) of Theorem 2.1 hold true, too.

Remark 2.3. The condition for a martingale to be a purely discontinuous one is of local nature. Therefore, the above theorem and corollary also hold true when considering (semi-)martingale laws defined on the time interval $[0, \infty)$, up to the slight modification that the right-hand side of (4) must then hold for all $T \in[0, \infty)$.

We can use Theorem 2.1 to obtain a necessary and sufficient criterion on $\Theta$ for $\mathfrak{P}_{\text {sem }}^{a c}(\Theta)\left(\Gamma_{0}\right)$ to be compact. To that end, we introduce the following condition.

Condition (J). A set $\Theta \subseteq \mathbb{R}^{d} \times \mathbb{S}_{+}^{d} \times \mathcal{L}$ satisfies Condition $(\mathrm{J})$ if

$$
\lim _{\delta \downarrow 0} \sup _{(b, c, F) \in \Theta} \int_{\{|x| \leq \delta\}}|x|^{2} F(d x)=0 .
$$

Moreover, denote

$\operatorname{proj}_{c}(\Theta):=\left\{c \in \mathbb{S}_{+}^{d} \mid\right.$ there exists $(b, F) \in \mathbb{R}^{d} \times \mathcal{L}$ such that $\left.(b, c, F) \in \Theta\right\}$,

$\operatorname{proj}_{F}(\Theta):=\left\{F \in \mathcal{L} \mid\right.$ there exists $(b, c) \in \mathbb{R}^{d} \times \mathbb{S}_{+}^{d}$ such that $\left.(b, c, F) \in \Theta\right\}$.

Definition 2.4. Given $\Theta \subseteq \mathbb{R}^{d} \times \mathbb{S}_{+}^{d} \times \mathcal{L}$, we say that $\operatorname{proj}_{c}(\Theta)$ does not depend on $\operatorname{proj}_{F}(\Theta)$ if for any fixed $F \in \operatorname{proj}_{F}(\Theta)$,

$\left\{c \in \operatorname{proj}_{c}(\Theta) \mid\right.$ there exists $b \in \mathbb{R}^{d}$ such that $\left.(b, c, F) \in \Theta\right\}=\operatorname{proj}_{c}(\Theta)$.

The condition on $\Theta$ introduced in Definition 2.4 can be seen as a generalization of a box. Indeed, for any subsets $D_{b} \subseteq \mathbb{R}^{d}, D_{c} \subseteq \mathbb{S}_{+}^{d}, D_{F} \subseteq \mathcal{L}$, the box $\Theta_{\text {Box }}:=$ $D_{b} \times D_{c} \times D_{F}$ satisfies the above condition. Let us state the compactness criterion for $\mathfrak{P}_{\text {sem }}^{a c}(\Theta)\left(\Gamma_{0}\right)$.

Theorem 2.5. Let $\Theta \subseteq \mathbb{R}^{d} \times \mathbb{S}^{d} \times \mathcal{L}$ be closed, convex, and satisfy Condition (B). Then the following hold true:

(1) $\Theta$ satisfying Condition $(\mathrm{J})$ implies closedness of $\mathfrak{P}_{\text {sem }}^{a c}(\Theta)$.

(2) On the other hand, if additionally $\Theta$ is satisfying the condition in Definition 2.4, then closedness of $\mathfrak{P}_{\text {sem }}^{a c}(\Theta)$ implies that $\Theta$ satisfies Condition $(\mathrm{J})$.

In addition, consider a set of distributions $\Gamma_{0} \subseteq \mathfrak{M}_{1}\left(\mathbb{R}^{d}\right)$ being compact. Then:

(3) $\Theta$ satisfying Condition $(\mathrm{J})$ implies compactness of $\mathfrak{P}_{\text {sem }}^{a c}(\Theta)\left(\Gamma_{0}\right)$.

(4) On the other hand, if additionally $\Theta$ is satisfying the condition in Definition 2.4, then compactness of $\mathfrak{P}_{\text {sem }}^{a c}(\Theta)\left(\Gamma_{0}\right)$ implies that $\Theta$ satisfies Condition $(\mathrm{J})$.

Remark 2.6. Under the above conditions, tightness of $\Gamma_{0} \subseteq \mathfrak{M}_{1}\left(\mathbb{R}^{d}\right)$ implies tightness of $\mathfrak{P}_{\text {sem }}^{a c}(\Theta)\left(\Gamma_{0}\right)$; see Corollary 3.22. Moreover, closedness of $\Gamma_{0}$ ensures that 
any limit law $\mathbb{P}_{0}$ of a sequence $\left(\mathbb{P}_{n}\right) \in \mathfrak{P}_{\text {sem }}^{a c}(\Theta)\left(\Gamma_{0}\right)$ satisfies $\mathbb{P}_{0} \circ X_{0}^{-1} \in \Gamma_{0}$. Therefore, parts (3) and (4) are simple consequences of parts (1) and (2).

Remark 2.7. By the same arguments, the results of Theorem 2.5 also hold true when considering semimartingale laws defined on the time interval $[0, \infty)$.

Next, we provide a second compactness criterion for $\mathfrak{P}_{\text {sem }}^{a c}(\Theta)\left(\Gamma_{0}\right)$ expressed by the infinitesimal generator of the Lévy laws in $\mathfrak{P}_{s e m}^{a c}(\Theta)$. To that end, for any set of Lévy triplets $\Theta$ set

$$
\mathfrak{P}_{L}(\Theta):=\left\{\mathbb{P} \in \mathfrak{P}_{\text {sem }}^{a c}(\Theta) \mid X \text { is a } \mathbb{P}-\mathbb{F} \text {-Lévy process }\right\} \text {. }
$$

For any $(b, c, F) \in \Theta$ we consider the infinitesimal generator $\mathfrak{L}^{(b, c, F)}$, which satisfies for any smooth enough function $f$ that

$$
\begin{aligned}
\left(\mathfrak{L}^{(b, c, F)} f\right)(x):= & \sum_{i=1}^{d} b^{i} \frac{\partial f}{\partial x^{i}}(x)+\frac{1}{2} \sum_{i, j=1}^{d} c^{i j} \frac{\partial^{2} f}{\partial x^{i} \partial x^{j}}(x) \\
& +\int_{\mathbb{R}^{d}}\left(f(x+y)-f(x)-\sum_{i=1}^{d} \frac{\partial f}{\partial x^{i}}(x) h^{i}(y)\right) F(d y),
\end{aligned}
$$

and introduce the Lévy exponent function $\psi^{(b, c, F)} \in C\left(\mathbb{R}^{d}, \mathbb{C}\right)$ given by

$$
\psi^{(b, c, F)}(x)=\mathrm{i} x \cdot b-\frac{1}{2} x \cdot c \cdot x+\int_{\mathbb{R}^{d}}\left(\mathrm{e}^{\mathrm{i} x \cdot y}-1-\mathrm{i} x \cdot h(y)\right) F(d y) .
$$

Furthermore, consider a function $u: \mathbb{R}^{d} \times \mathbb{S}_{+}^{d} \times \mathcal{L} \rightarrow \mathbb{R}^{d} \times \mathbb{S}_{+}^{d} \times \mathcal{L}$ defined by

$$
(b, c, F) \mapsto(b, \widetilde{c}, F), \quad \text { where } \quad \widetilde{c}^{i j}=c^{i j}+\int_{\mathbb{R}^{d}} h^{i}(x) h^{j}(x) F(d x) .
$$

Denote by $C_{b}^{2}\left(\mathbb{R}^{d}\right)$ the set of bounded continuous functions with bounded continuous derivatives up to the second order. Then we obtain the following second compactness criterion for semimartingale laws $\mathfrak{P}_{\text {sem }}^{a c}(\Theta)\left(\Gamma_{0}\right)$.

Theorem 2.8. Let $\Theta \subset \mathbb{R}^{d} \times \mathbb{S}_{+}^{d} \times \mathcal{L}$ be closed, convex, and satisfy condition (B). Then the following are equivalent:

(1) $u(\Theta) \subseteq \mathbb{R}^{d} \times \mathbb{S}_{+}^{d} \times \mathcal{L}$ is closed.

(2) For any compact set $\Gamma_{0} \subseteq \mathfrak{M}_{1}\left(\mathbb{R}^{d}\right)$ of distributions on $\mathbb{R}^{d}$, the set of laws $\mathfrak{P}_{\text {sem }}^{a c}(\Theta)\left(\Gamma_{0}\right)$ is compact.

(3) $\mathfrak{P}_{L}(\Theta)$ is compact.

(4) The set $\left\{\mathfrak{L}^{(b, c, F)}:(b, c, F) \in \Theta\right\}$ is sequentially compact with respect to $C_{b}^{2}\left(\mathbb{R}^{d}\right)$-test functions for the topology of pointwise convergence. More precisely, for every sequence $\left(\left(b^{n}, c^{n}, F^{n}\right)\right)_{n \in \mathbb{N}} \subseteq \Theta$ there exists a subsequence $\left(\left(b^{n_{k}}, c^{n_{k}}, F^{n_{k}}\right)\right)_{k \in \mathbb{N}}$ and $\left(b^{0}, c^{0}, F^{0}\right) \in \Theta$ such that for all $f \in C_{b}^{2}\left(\mathbb{R}^{d}\right)$ the subsequence of functions $\left(\left(\mathfrak{L}^{\left(b^{n_{k}}, c^{n_{k}}, F^{n_{k}}\right)} f\right)\right)_{k \in \mathbb{N}}$ converges pointwise to the function $\left(\mathfrak{L}^{\left(b^{0}, c^{0}, F^{0}\right)} f\right)$.

(5) The set of functions $\left\{\psi^{(b, c, F)}:(b, c, F) \in \Theta\right\}$ is sequentially compact for the topology of pointwise convergence. More precisely, for every sequence $\left(\left(b^{n}, c^{n}, F^{n}\right)\right)_{n \in \mathbb{N}} \subseteq \Theta$ there exists a subsequence $\left(\left(b^{n_{k}}, c^{n_{k}}, F^{n_{k}}\right)\right)_{k \in \mathbb{N}}$ and $\left(b^{0}, c^{0}, F^{0}\right) \in \Theta$ such that the subsequence of functions $\left(\psi^{\left(b^{n_{k}}, c^{n_{k}}, F^{n_{k}}\right)}\right)_{k \in \mathbb{N}}$ converges pointwise to the function $\psi^{\left(b^{0}, c^{0}, F^{0}\right)}$.

Remark 2.9. Since conditions (1), (4), and (5) are local conditions, Theorem 2.8 remains valid for semimartingale laws defined on the time interval $[0, \infty)$. 
Remark 2.10. For most sets of Lévy triplets $\Theta$, if one wants to check whether one of the equivalent conditions in Theorem 2.8 to $\mathfrak{P}_{\text {sem }}^{a c}(\Theta)\left(\Gamma_{0}\right)$ being compact holds true, one needs to verify that any sequence of (sums of compensated) small jumps cannot create an additional diffusion in the limit. This immediately links to the results obtained in Theorems 2.1 and 2.5. In particular, Theorem 2.8 cannot replace Theorems 2.1 and 2.5. which are much deeper results, as they explain how one can prevent purely discontinuous martingales from creating an additional diffusion in the limit, which then leads to a compactness criterion expressed through Condition $(\mathrm{J})$. However, if roughly speaking, $\Theta$ is described by a boundedness condition on the modified second differential characteristic (cf. e.g. Example 2.11), then condition (1) in Theorem 2.8 can be verified directly. The intuition why this holds is the following.

A semimartingale is described by its drift, its diffusion, its sum of big jumps, and its sum of compensated small jumps. Notice that drifts converge to a drift, diffusions converge to a diffusion, and the sum of big jumps converges to a sum of big jumps; we refer to Subsection 3.2 for a precise statement. However, a sequence of sums of compensated small jumps converges to a sum of compensated small jumps and a possible additional diffusion. This is why if no additional diffusion may be created in the limit, then closedness of $\Theta$ implies closedness of $\mathfrak{P}_{\text {sem }}^{a c}(\Theta)\left(\Gamma_{0}\right)$, and hence also compactness of $\mathfrak{P}_{s e m}^{a c}(\Theta)\left(\Gamma_{0}\right)$, due to $\Theta$ satisfying Condition (B). But notice that the modified second characteristic is defined as the sum of the (quadratic variation of the) diffusion and the (compensator of the sum of the squared) small jumps. Therefore, a sequence of modified second characteristics converges to the modified second characteristic of the limit process. Thus, if $\Theta$ is described by a constraint only on the modified second differential characteristic, without any constraints on the second differential characteristic, then condition (1) in Theorem 2.8 can be verified directly due to the definition of the map $u$ involved in condition (1).

The proofs of Theorems 2.1, 2.5, and 2.8 are given in Section 4. Now, we present an example showing that in Theorem 2.5 . Condition $(\mathrm{J})$ is not necessary for $\mathfrak{P}_{\text {sem }}^{a c}(\Theta)\left(\Gamma_{0}\right)$ to be compact.

Example 2.11. Let the dimension be $d=1$. For any $F \in \mathcal{L}$ denote by $\operatorname{supp}(F)$ the support of the measure $F$ and define $\Theta \subseteq \mathbb{R} \times[0, \infty) \times \mathcal{L}$ by

$$
\Theta:=\left\{\left.(b, c, F)\left|\operatorname{supp}(F) \subseteq\{|x| \leq 1\}, b=0, c+\int_{\mathbb{R}}\right| x\right|^{2} F(d x)=1\right\},
$$

where the (differential) characteristics are defined with respect to a continuous truncation function $h$ satisfying $h(x)=x$ on $\{|x| \leq 1\}$. Observe that $\Theta$ does not satisfy the condition in Definition 2.4. It turns out that $\Theta$ is closed, convex, and satisfies Condition (B), but Condition $(J)$ fails. However, the set $\mathfrak{P}_{\text {sem }}^{a c}(\Theta)\left(\left\{\delta_{0}\right\}\right)$ is compact. Compactness of $\mathfrak{P}_{\text {sem }}^{a c}(\Theta)\left(\left\{\delta_{0}\right\}\right)$ is shown by verifying that condition (1) in Theorem 2.8 holds true. We provide the proof at the end of Section 4 .

Remark 2.12. As mentioned above, we know from [31, Proposition 2.2] that the precise choice of the filtration in the definition of the set $\mathfrak{P}_{\text {sem }}$ and its subsets introduced above is not crucial in the sense that the results above also hold true with respect to any filtration $\mathbb{F} \subseteq \mathbb{G} \subseteq \mathbb{F}_{+}^{\mathbb{P}}$.

Remark 2.13. Even though $\mathfrak{P}_{\text {sem }}^{a c}(\Theta) \neq \emptyset$, we might have that $\mathfrak{P}_{m}^{a c}(\Theta)=\emptyset$ or $\mathfrak{P}_{m, d}^{a c}(\Theta)=\emptyset$. However, one can easily impose conditions (additional to the one in 
Theorem 2.11) on $\Theta$ such that both sets above are nonempty. In fact, if in addition to Condition (B) we assume that

$$
\Theta \subseteq\left\{(b, c, F) \in \mathbb{R}^{d} \times \mathbb{S}_{+}^{d} \times \mathcal{L} \mid b+\int_{\mathbb{R}^{d}}[x-h(x)] F(d x)=0\right\}=: \Theta_{\mathcal{M}},
$$

then we have $\left\{\mathbb{P} \in \mathfrak{P}_{\text {sem }}^{a c}(\Theta) \mid \mathbb{E}^{\mathbb{P}}\left[\left|X_{0}\right|\right]<\infty\right\}=\mathfrak{P}_{m}^{a c}(\Theta)$. Moreover, if we impose that

$$
\Theta \subseteq\left\{(b, c, F) \in \Theta_{\mathcal{M}} \mid c=0\right\}=: \Theta_{\mathcal{M}^{d}}
$$

then we even obtain $\left\{\mathbb{P} \in \mathfrak{P}_{\text {sem }}^{a c}(\Theta) \mid \mathbb{E}^{\mathbb{P}}\left[\left|X_{0}\right|\right]<\infty\right\}=\mathfrak{P}_{m, d}^{a c}(\Theta)$.

As an application of Theorem 2.5. we consider the following semimartingale optimal transportation problem. First, let $T=1$ and fix any set $\Theta \subseteq \mathbb{R}^{d} \times \mathbb{S}^{d} \times \mathcal{L}$ to be closed, convex, and satisfying Conditions (B) and (J). To shorten the notation, we write $\mathfrak{P}_{\Theta} \equiv \mathfrak{P}_{\text {sem }}^{a c}(\Theta)$. Given two arbitrary probability measures $\mu_{0}$ and $\mu_{1}$ in $\mathfrak{M}_{1}\left(\mathbb{R}^{d}\right)$, we denote

$$
\begin{gathered}
\mathfrak{P}_{\Theta}\left(\mu_{0}\right):=\left\{\mathbb{P} \in \mathfrak{P}_{\Theta} \mid \mathbb{P} \circ X_{0}^{-1}=\mu_{0}\right\}, \\
\mathfrak{P}_{\Theta}\left(\mu_{0}, \mu_{1}\right):=\left\{\mathbb{P} \in \mathfrak{P}_{\Theta}\left(\mu_{0}\right) \mid \mathbb{P} \circ X_{1}^{-1}=\mu_{1}\right\} .
\end{gathered}
$$

The semimartingale $X$ under $\mathbb{P} \in \mathfrak{P}_{\Theta}\left(\mu_{0}, \mu_{1}\right)$ can be viewed as a medium of mass transportation from the initial distribution $\mu_{0}$ to the target distribution $\mu_{1}$. We couple $\mathbb{P}$ with a transportation cost

$$
\mathfrak{J}(\mathbb{P}):=\mathbb{E}^{\mathbb{P}}\left[\int_{0}^{1} L\left(t, X, b_{t}^{\mathbb{P}}, c_{t}^{\mathbb{P}}, F_{t}^{\mathbb{P}}\right) d t\right],
$$

where $L:[0,1] \times \Omega \times \Theta \rightarrow[0, \infty)$ is a given cost function. Then, we are interested in the optimal transport problem

$$
V\left(\mu_{0}, \mu_{1}\right):=\inf _{\mathbb{P} \in \mathfrak{P}_{\Theta}\left(\mu_{0}, \mu_{1}\right)} \mathfrak{J}(\mathbb{P}),
$$

using the convention $\inf \emptyset=\infty$. The goal is to prove a duality result for the minimizing problem (6), which can be seen as an extension of the classical Kantorovich duality in optimal transportation. To that end, we need some conditions on $L$.

Assumption 2.14. The cost function $L:(t, \omega, \theta) \in[0,1] \times \Omega \times \Theta \mapsto L(t, \omega, \theta) \in$ $[0, \infty)$ satisfies:

(1) $L$ is nonnegative, continuous in $(t, \omega, \theta)$, and convex in $\theta$.

(2) $L$ is uniformly continuous in $t$ in the sense that

$$
\Delta_{t} L(\epsilon):=\sup _{0 \leq s, t \leq 1,|t-s|<\epsilon, \omega \in \Omega, \theta \in \Theta} \frac{|L(s, \omega, \theta)-L(t, \omega, \theta)|}{1+L(t, \omega, \theta)} \longrightarrow 0 \quad \text { as } \varepsilon \rightarrow 0 .
$$

Remark 2.15. In the case $\Theta=(U \times\{0\})$ where $U \subseteq \mathbb{R}^{d} \times \mathbb{S}_{+}^{d}$ and here 0 denotes the zero-measure $\in \mathcal{L}$, conditions (1) and (2) coincide with [39, Assumptions 3.1 and 3.2]. In fact, when $U=\left\{I_{d \times d}\right\} \times \mathbb{R}^{d}$, assumption (2) coincides with 30, Assumption A.1].

We define the dual formulation of (6) by

$$
\mathcal{V}\left(\mu_{0}, \mu_{1}\right):=\sup _{\lambda_{1} \in C_{b}\left(\mathbb{R}^{d}\right)}\left\{\int_{\mathbb{R}^{d}} \lambda_{0}^{\lambda_{1}}(x) \mu_{0}(d x)-\int_{\mathbb{R}^{d}} \lambda_{1}(x) \mu_{1}(d x)\right\},
$$


where

$$
\lambda_{0}^{\lambda_{1}}(x):=\inf _{\mathbb{P} \in \mathfrak{P}_{\Theta}\left(\delta_{x}\right)} \mathbb{E}^{\mathbb{P}}\left[\int_{0}^{1} L\left(t, X, b_{t}^{\mathbb{P}}, c_{t}^{\mathbb{P}}, F_{t}^{\mathbb{P}}\right) d t+\lambda_{1}\left(X_{1}\right)\right] .
$$

The integral $\int_{\mathbb{R}^{d}} \lambda_{0}^{\lambda_{1}}(x) \mu_{0}(d x)$ is well-defined as the function $\lambda_{0}^{\lambda_{1}}$ is bounded from below (might take value $\infty$ ) and is measurable; cf. Lemma 5.4. Then, our main duality result is the following.

Theorem 2.16. Let $\Theta \subseteq \mathbb{R}^{d} \times \mathbb{S}_{+}^{d} \times \mathcal{L}$ be closed, convex, and satisfy Conditions (B) and (J). Moreover, let the cost function L satisfy Assumption 2.14. Then

$$
V\left(\mu_{0}, \mu_{1}\right)=\mathcal{V}\left(\mu_{0}, \mu_{1}\right), \quad \text { for all } \mu_{0}, \mu_{1} \in \mathfrak{M}_{1}\left(\mathbb{R}^{d}\right),
$$

and the infimum is achieved by some $\widehat{\mathbb{P}} \in \mathfrak{P}_{\Theta}\left(\mu_{0}, \mu_{1}\right)$ for the primal problem $V\left(\mu_{0}, \mu_{1}\right)$ defined in (6) whenever it is finite.

Remark 2.17. In general, $\mathfrak{P}_{\Theta}\left(\mu_{0}, \mu_{1}\right)$ might be empty. However, for example when $\mu_{0}=\delta_{x}$ for some $x \in \mathbb{R}^{d}$ and $\mu_{1}$ is some suitable infinitely divisible distribution (suitable with respect to $\Theta$ ), then $\mathfrak{P}_{\Theta}\left(\mu_{0}, \mu_{1}\right)$ is clearly nonempty containing at least the corresponding Lévy law. Moreover, $\mathfrak{P}_{\Theta}\left(\mu_{0}, \mu_{1}\right)$ being nonempty is closely related to the Skorokhod Embedding Problem for Lévy processes with nontrivial initial law.

\section{A version of Theorems 2.1 ANd 2.5 on AN ENLARGed SPACE}

3.1. An enlarged space. In this subsection, we introduce an enlarged space. The strategy of the proofs of our main results is to derive them first in the enlarged space and then conclude for the original space.

Let $\mathcal{C}^{+}\left(\mathbb{R}^{d}\right):=\left\{g_{i} \mid i \in \mathbb{N}\right\}$ be a countable family of bounded continuous functions on $\mathbb{R}^{d}$ vanishing in a neighborhood of the origin with the following properties:

- It is a law-determining class for Lévy measures; i.e., for any two Lévy measures $F, F^{\prime} \in \mathcal{L}$, if one has $\int_{\mathbb{R}^{d}} g(x) F(d x)=\int_{\mathbb{R}^{d}} g(x) F^{\prime}(d x)$ for all $g \in \mathcal{C}^{+}\left(\mathbb{R}^{d}\right)$, then $F=F^{\prime}$.

- It is a convergence-determining class for the weak convergence induced by the bounded continuous functions on $\mathbb{R}^{d}$ vanishing in a neighborhood of the origin; i.e., given any sequence $\left(F_{n}\right)_{n \in \mathbb{N}} \subseteq \mathcal{L}$ and $F \in \mathcal{L}$, if

$$
\lim _{n \rightarrow \infty} \int_{\mathbb{R}^{d}} g(x) F_{n}(d x)=\int_{\mathbb{R}^{d}} g(x) F(d x),
$$

for all $g \in \mathcal{C}^{+}\left(\mathbb{R}^{d}\right)$, then $\lim _{n \rightarrow \infty} \int_{\mathbb{R}^{d}} g(x) F_{n}(d x)=\int_{\mathbb{R}^{d}} g(x) F(d x)$ for all bounded continuous functions on $\mathbb{R}^{d}$ vanishing in a neighborhood of the origin.

- For each $m \in \mathbb{N}$, let $g_{2 m}$ be a continuous function on $\mathbb{R}^{d}$ satisfying for all $x \in \mathbb{R}^{d}$ that $0 \leq g_{2 m}(x) \leq|x|^{2} \wedge 1$ and that

$$
g_{2 m}(x)= \begin{cases}0 & \text { if }|x| \leq \frac{1}{2 m} \\ |x|^{2} \wedge 1 & \text { if }|x|>\frac{1}{m} .\end{cases}
$$

We refer to [21, II.2.20] together with [21, VII.2.7 and 2.8] for the existence of such a class $\mathcal{C}^{+}\left(\mathbb{R}^{d}\right)$.

For any $n \in \mathbb{N}$, write $\Omega_{n}=\mathbb{D}\left([0, T], \mathbb{R}^{n}\right)$ endowed with the Skorokhod topology, and $\Omega_{n}^{c}:=C\left([0, T], \mathbb{R}^{n}\right)$ for the space of continuous function with the usual uniform 
topology. Recall that $\Omega=\Omega_{d}=\mathbb{D}\left([0, T], \mathbb{R}^{d}\right)$ is our original space. We introduce the enlarged space

$$
\bar{\Omega}:=\Omega \times \Omega_{d}^{c} \times \Omega_{d}^{c} \times \Omega \times \Omega \times \Omega_{d^{2}}^{c} \times \Omega_{d^{2}} \times \Omega_{d^{2}}^{c} \times\left(\Omega_{1} \times \Omega_{1}^{c}\right)^{\mathbb{N}},
$$

endowed with the product topology, becoming a Polish space. We write

$$
\overline{\mathbb{X}}:=\left(\bar{X}, \bar{B}, \bar{M}^{c}, \bar{M}^{d}, \bar{J}, \bar{C}, \overline{[M]}, \overline{\widetilde{C}},\left(\bar{U}^{i}, \bar{V}^{i}\right)_{i \in \mathbb{N}}\right)
$$

for the canonical process $\overline{\mathbb{X}}_{t}(\bar{\omega})=\bar{\omega}(t), \bar{\omega} \in \bar{\Omega}$. We endow $\bar{\Omega}$ with its Borel $\sigma$-field $\overline{\mathcal{F}}$ and denote by $\overline{\mathbb{F}}=\left(\overline{\mathbb{F}}_{t}\right)_{0 \leq t \leq T}$ the (raw) filtration generated by $\overline{\mathbb{X}}$.

Denote by $\overline{\mathfrak{P}}_{\text {sem }}^{a c}$ the set of all probability measures on $(\bar{\Omega}, \overline{\mathbb{F}})$ such that $\bar{X}$ is a $\overline{\mathbb{P}}$ - $\overline{\mathbb{F}}$-semimartingale with absolutely continuous characteristics having canonical representation

$$
\bar{X}-\bar{X}_{0}=\bar{M}^{c}+\bar{M}^{d}+\bar{B}+\bar{J} \quad \overline{\mathbb{P}} \text {-a.s., satisfying: }
$$

- $\bar{M}_{0}^{c}=\bar{M}_{0}^{d}=\bar{B}_{0}=0 \overline{\mathbb{P}}$-a.s.

- $\bar{B}$ has $\overline{\mathbb{P}}$-a.s. finite variation paths.

- $\bar{M}^{c}$ is a continuous $\overline{\mathbb{P}}-\overline{\mathbb{F}}$-local martingale with quadratic covariation $\bar{C}$.

- $\bar{M}^{d}$ is a purely discontinuous $\overline{\mathbb{P}}-\overline{\mathbb{F}}$-local martingale.

- $\bar{J}=\sum_{0 \leq s \leq .}\left[\Delta \bar{X}_{s}-h\left(\Delta \bar{X}_{s}\right)\right] \overline{\mathbb{P}}$-a.s.

- $\overline{\widetilde{C}}$ is the modified second characteristic of $\bar{X}$ under $\overline{\mathbb{P}}-\overline{\mathbb{F}}$ (for definition, see e.g. [21, p. 79]).

- $\overline{[M]}$ is the quadratic covariation of the local martingale $\bar{M}:=\bar{M}^{c}+\bar{M}^{d}$.

- $\overline{\mathbb{P}}$-a.s., each $\bar{U}^{i}$ coincides with $\int_{0}^{\cdot} \int_{\mathbb{R}^{d}} g_{i}(x) \mu^{\bar{X}}(d x, d t)$ (writing $g_{i}(x) * \mu^{\bar{X}}$ for brevity), and each $\bar{V}^{i}$ coincides with $g_{i}(x) * \nu^{\overline{\mathbb{P}}}$, where $\nu^{\overline{\mathbb{P}}}(d x, d t)$ denotes the $\overline{\mathbb{P}}-\overline{\mathbb{F}}$-compensator of the measure $\mu^{\bar{X}}(d x, d t)$ associated to the jumps of $\bar{X}$, and $g_{i}$ denotes the $i$-th element in $\mathcal{C}^{+}\left(\mathbb{R}^{d}\right)$.

We also define a slightly bigger class $\overline{\mathfrak{P}}_{s e m}^{a c, w}$ of probability measures which contains all laws $\overline{\mathbb{P}}$ on $(\bar{\Omega}, \overline{\mathcal{F}})$ satisfying the same conditions as above to be in $\overline{\mathfrak{P}}_{\text {sem }}^{\text {ac }}$, up to $(*)$, where we instead impose the weaker condition on $\bar{M}^{d}$ to be a $\overline{\mathbb{P}}$ - $\overline{\mathbb{F}}$-local martingale (but not necessarily a purely discontinuous one). Moreover, for any $\overline{\mathbb{P}} \in \overline{\mathfrak{P}}_{\text {sem }}^{a c}$ denote by $\left(\bar{b}^{\overline{\mathbb{P}}}, \bar{c}^{\overline{\mathbb{P}}}, \bar{F}^{\overline{\mathbb{P}}}\right)$ the differential characteristics of $\bar{X}$ with respect to $\overline{\mathbb{P}}-\overline{\mathbb{F}}$. Then, given $\Theta \subseteq \mathbb{R}^{d} \times \mathbb{S}_{+}^{d} \times \mathcal{L}$ and $\Gamma_{0} \subseteq \mathfrak{M}_{1}\left(\mathbb{R}^{d}\right)$, define $\overline{\mathfrak{P}}_{\text {sem }}^{a c}(\Theta)$ in accord with the definition of the corresponding sets on the original space $\Omega$ by

$$
\begin{aligned}
\overline{\mathfrak{P}}_{\text {sem }}^{a c}(\Theta) & :=\left\{\overline{\mathbb{P}} \in \overline{\mathfrak{P}}_{\text {sem }}^{a c} \mid\left(\bar{b}^{\overline{\mathbb{P}}}, \bar{c}^{\overline{\mathbb{P}}}, \bar{F}^{\overline{\mathbb{P}}}\right) \in \Theta \overline{\mathbb{P}} \times d t \text {-a.e. }\right\}, \\
\overline{\mathfrak{P}}_{\text {sem }}^{a c}(\Theta)\left(\Gamma_{0}\right) & :=\left\{\overline{\mathbb{P}} \in \overline{\mathfrak{P}}_{\text {sem }}^{a c}(\Theta) \mid \overline{\mathbb{P}} \circ \bar{X}_{0}^{-1} \in \Gamma_{0}\right\} .
\end{aligned}
$$

Next, we introduce a function $\bar{\varphi}: \mathbb{R}^{d} \times \mathbb{S}_{+}^{d} \times \mathcal{L} \rightarrow \mathbb{R}^{d} \times \mathbb{S}_{+}^{d} \times \mathbb{R}^{\mathbb{N}}$, which turns out to be useful for proving that the differential characteristics under a limit law $\overline{\mathbb{P}}_{0}$ of a sequence $\left(\overline{\mathbb{P}}_{n}\right) \subseteq \overline{\mathfrak{P}}_{\text {sem }}^{a c}(\Theta)$ are taking values in $\Theta$. Let $\left\{g_{i} \mid i \in \mathbb{N}\right\}=\mathcal{C}^{+}\left(\mathbb{R}^{d}\right)$ and define first an additive, positive homogeneous function $\varphi: \mathcal{L} \rightarrow \mathbb{R}^{\mathbb{N}}$ via

$$
\varphi(F):=\left(\int_{\mathbb{R}^{d}} g_{i}(x) F(d x)\right)_{i \in \mathbb{N}} .
$$


We deduce from $\mathcal{C}^{+}\left(\mathbb{R}^{d}\right)$ being a law-determining class on $\mathcal{L}$ that $\varphi$ is injective. Now, define $\bar{\varphi}: \mathbb{R}^{d} \times \mathbb{S}_{+}^{d} \times \mathcal{L} \rightarrow \mathbb{R}^{d} \times \mathbb{S}_{+}^{d} \times \mathbb{R}^{\mathbb{N}}$ by

$$
(b, c, F) \mapsto(b, c, \varphi(F)) .
$$

Clearly, $\bar{\varphi}$ is additive, positive homogeneous, and also a bijection onto its image. Mostly, we will use the function $\bar{\varphi}$ in the following way: Define the processes

$$
\bar{b}_{t}:=\limsup _{n \rightarrow \infty} n\left(\bar{B}_{t}-\bar{B}_{\left(t-\frac{1}{n}\right) \vee 0}\right), \quad \bar{c}_{t}:=\limsup _{n \rightarrow \infty} n\left(\bar{C}_{t}-\bar{C}_{\left(t-\frac{1}{n}\right) \vee 0}\right), \quad t \in[0, T],
$$

as well as the sequence of processes $\bar{v}_{t}:=\left(\bar{v}_{t}^{1}, \bar{v}_{t}^{2}, \ldots\right)$ by setting for each $i \in \mathbb{N}$,

$$
\bar{v}_{t}^{i}:=\limsup _{n \rightarrow \infty} n\left(\bar{V}_{t}^{i}-\bar{V}_{\left(t-\frac{1}{n}\right) \vee 0}^{i}\right), \quad t \in[0, T] .
$$

Whenever $\overline{\mathbb{P}} \in \overline{\mathfrak{P}}_{\text {sem }}^{\text {ac }}$, we have

$$
\left(\bar{b}_{t}^{\overline{\mathbb{P}}}, \bar{c}_{t}^{\overline{\mathbb{P}}},\left(\int_{\mathbb{R}^{d}} g_{i}(x) \bar{F}_{t}^{\overline{\mathbb{P}}}(d x)\right)_{i \in \mathbb{N}}\right)=\left(\bar{b}_{t}, \bar{c}_{t},\left(\bar{v}_{t}^{i}\right)_{i \in \mathbb{N}}\right) \quad \overline{\mathbb{P}} \times d t \text {-a.s. }
$$

Therefore, for any given $\Theta \subseteq \mathbb{R}^{d} \times \mathbb{S}_{+}^{d} \times \mathcal{L}$ and $\overline{\mathbb{P}} \in \overline{\mathfrak{P}}_{\text {sem }}^{a c}$ we have

$$
\left(\bar{b}^{\overline{\mathbb{P}}}, \bar{c}^{\overline{\mathbb{P}}}, \bar{F}^{\overline{\mathbb{P}}}\right) \in \Theta \overline{\mathbb{P}} \times d t \text {-a.s. } \Longleftrightarrow(\bar{b}, \bar{c}, \bar{v}) \in \bar{\varphi}(\Theta) \overline{\mathbb{P}} \times d t \text {-a.s. }
$$

In addition, note that as $\mathcal{C}^{+}\left(\mathbb{R}^{d}\right)$ is a convergence-determining class for the weak convergence induced by the bounded continuous functions on $\mathbb{R}^{d}$ vanishing in a neighborhood of the origin, we see from (10) that for any given $\Theta \subseteq \mathbb{R}^{d} \times \mathbb{S}_{+}^{d} \times \mathcal{L}$ which is closed and $\overline{\mathbb{P}} \in \overline{\mathfrak{P}}_{\text {sem }}^{a c}$ we have

$$
\left(\bar{b}^{\overline{\mathbb{P}}}, \bar{c}^{\overline{\mathbb{P}}}, \bar{F}^{\overline{\mathbb{P}}}\right) \in \Theta \overline{\mathbb{P}} \times d t \text {-a.s. } \Longleftrightarrow(\bar{b}, \bar{c}, \bar{v}) \in \operatorname{cl}(\bar{\varphi}(\Theta)) \quad \overline{\mathbb{P}} \times d t \text {-a.s. },
$$

where $\operatorname{cl}(\bar{\varphi}(\Theta))$ denotes the closure of $\bar{\varphi}(\Theta) \subseteq \mathbb{R}^{d} \times \mathbb{S}_{+}^{d} \times \mathbb{R}^{\mathbb{N}}$.

The main goal of Section 3 is to formulate and prove the following proposition, which is a version of Theorem 2.1 on the enlarged space $\bar{\Omega}$. Recall that under each $\overline{\mathbb{P}} \in \overline{\mathfrak{P}}_{\text {sem }}^{a c}, \bar{M}^{d}$ is a purely discontinuous local martingale.

Proposition 3.1. Let $\Theta \subseteq \mathbb{R}^{d} \times \mathbb{S}_{+}^{d} \times \mathcal{L}$ satisfy Condition (B) and let $\left(\overline{\mathbb{P}}_{n}\right)_{n \in \mathbb{N}} \subseteq$ $\overline{\mathfrak{P}}_{\text {sem }}^{\text {ac }}(\Theta)$ be a sequence converging weakly to some law $\overline{\mathbb{P}}_{0} \in \mathfrak{M}_{1}(\bar{\Omega})$. Then, the following hold true:

(1) $\bar{M}^{d}$ is a $\overline{\mathbb{P}}_{0}-\overline{\mathbb{F}}$-martingale.

(2) We have the following necessary and sufficient criterion for $\bar{M}^{d}$ being a purely discontinuous martingale under $\overline{\mathbb{P}}_{0}-\overline{\mathbb{F}}$ :

$$
\begin{gathered}
\bar{M}^{d} \text { is a purely discontinuous } \overline{\mathbb{P}}_{0}-\overline{\mathbb{F}} \text {-martingale } \\
\Longleftrightarrow \lim _{\delta \downarrow 0} \limsup _{n \rightarrow \infty} \mathbb{E}^{\overline{\mathbb{P}}_{n}}\left[\int_{0}^{T} \int_{\{|x| \leq \delta\}}|x|^{2} \bar{F}_{t} \overline{\mathbb{P}}_{n}(d x) d t\right]=0 .
\end{gathered}
$$

As a consequence of Proposition 3.1 we obtain a closedness criterion for $\overline{\mathfrak{P}}_{\text {sem }}^{a c}(\Theta)$.

Corollary 3.2. Let $\Theta \subseteq \mathbb{R}^{d} \times \mathbb{S}_{+}^{d} \times \mathcal{L}$ satisfy Conditions $(\mathrm{B})$ and $(\mathrm{J})$. Consider a sequence $\left(\overline{\mathbb{P}}_{n}\right) \subseteq \overline{\mathfrak{P}}_{\text {sem }}^{\text {ac }}(\Theta)$ converging weakly to some $\overline{\mathbb{P}}_{0} \in \mathfrak{M}_{1}(\bar{\Omega})$. The following hold:

(1) We have $\overline{\mathbb{P}}_{0} \in \overline{\mathfrak{P}}_{\text {sem }}^{a c}$.

(2) If in addition, $\Theta$ is closed and convex, then $\overline{\mathbb{P}}_{0} \in \overline{\mathfrak{P}}_{\text {sem }}^{a c}(\Theta)$. In particular, $\overline{\mathfrak{P}}_{\text {sem }}^{a c}(\Theta)$ is closed. 
One of the key techniques to prove Proposition 3.1 and Corollary 3.2 is the Skorokhod representation theorem [36] (see also 22]), which states the following.

Theorem 3.3 (Skorokhod representation theorem). There exists a sequence of $\bar{\Omega}$ valued random variables $\left(z^{n}\right)_{n \in \mathbb{N}_{0}}$ defined on $([0,1], \mathcal{B}([0,1]), \lambda)$, where $\lambda$ denotes the Lebesgue measure, such that for each $n \in \mathbb{N}_{0}, \overline{\mathbb{P}}_{n}=\lambda \circ\left(z^{n}\right)^{-1}$ and $\left(z^{n}\right)$ converges to $z^{0}$ with respect to the product topology on $\bar{\Omega}$.

Denote by $z^{n, \bar{X}}:=\bar{X} \circ z^{n} \in \Omega$, and define in the same way $z^{n, \bar{B}}, \ldots, z^{n, \bar{C}}$ as well as $z^{n, \bar{U}^{i}}, z^{n, \bar{V}^{i}}$ for each $i \in \mathbb{N}$. The convergence of $\left(z^{n}\right)$ to $z^{0}$ on $\bar{\Omega}$ implies the convergence of each sequence $\left(z^{n, \bar{X}}\right), \ldots,\left(z^{n, \bar{V}^{i}}\right)$ to $z^{0, \bar{X}}, \ldots, z^{0, \bar{V}^{i}}$, respectively, on the corresponding space.

The proofs of Proposition 3.1 and Corollary 3.2 are divided into several lemmas, provided in the following subsections. For standard notation appearing in the theory of weak convergence of processes, we refer to 21 .

3.2. Semimartingale property of $\bar{X}$ under the limit law $\overline{\mathbb{P}}_{0}$. The main objective of this subsection is to prove that any limit law $\overline{\mathbb{P}}_{0}$ of a sequence $\left(\overline{\mathbb{P}}_{n}\right)_{n \in \mathbb{N}} \subseteq$ $\overline{\mathfrak{P}}_{\text {sem }}^{a c}(\Theta)$ with $\Theta$ satisfying Condition $(\mathrm{B})$ is an element of $\overline{\mathfrak{P}}_{\text {sem }}^{a c, w}$. In particular, $\bar{X}$ has no fixed time of discontinuity.

Proposition 3.4. Let $\Theta \subseteq \mathbb{R}^{d} \times \mathbb{S}_{+}^{d} \times \mathcal{L}$ satisfy Condition (B) and let $\left(\overline{\mathbb{P}}_{n}\right)_{n \in \mathbb{N}} \subseteq$ $\overline{\mathfrak{P}}_{\text {sem }}^{\text {ac }}(\Theta)$ be a sequence converging weakly to some law $\overline{\mathbb{P}}_{0}$. Then, $\overline{\mathbb{P}}_{0} \in \overline{\mathfrak{P}}_{\text {sem }}^{a c, w}$. In addition, we have

(i) $\mathbb{E}^{\overline{\mathbb{P}}_{0}}\left[\int_{0}^{T}\left[\left|\overline{\bar{P}}_{s}\right|+\left|\bar{c}_{s}^{\overline{\mathbb{P}}_{0}}\right|+\int_{\mathbb{R}^{d}}|h(x)|^{2} \bar{F}_{s}^{\overline{\mathbb{P}}_{0}}(d x)\right] d s\right]<\infty$.

(ii) Both $\bar{M}^{c}$ and $\bar{M}^{d}$ are $\overline{\mathbb{P}}_{0}-\overline{\mathbb{F}}$-martingales.

This is the first step towards the proofs of Proposition 3.1 and Corollary 3.2 The proof of Proposition 3.4 is divided into several lemmas. We will frequently use the fact that for any continuous function $f: \mathbb{R}^{d} \rightarrow \mathbb{R}^{m}$ vanishing on a neighborhood of 0 , the map

$$
I^{f}: \mathbb{D}\left([0, T] ; \mathbb{R}^{d}\right) \rightarrow \mathbb{D}\left([0, T] ; \mathbb{R}^{m}\right), \quad \alpha \mapsto I^{f}(\alpha):=\sum_{0 \leq s \leq .} f\left(\Delta \alpha_{s}\right)
$$

is continuous; see [21, Corollary VI.2.8, p. 340].

Lemma 3.5. Let $\left(\overline{\mathbb{P}}_{n}\right)_{n \in \mathbb{N}} \subseteq \overline{\mathfrak{P}}_{\text {sem }}^{a c}$ be a sequence converging weakly to some law $\overline{\mathbb{P}}_{0} \in \mathfrak{M}_{1}(\bar{\Omega})$. Then we have:

(i) $\bar{J}=\sum_{0 \leq s \leq .}\left[\Delta \bar{X}_{s}-h\left(\Delta \bar{X}_{s}\right)\right] \overline{\mathbb{P}}_{0}$-a.s.

(ii) For each $i \in \mathbb{N}$, we have $\bar{U}^{i}=\sum_{0 \leq s \leq .} g_{i}\left(\Delta X_{s}\right) \overline{\mathbb{P}}_{0}$-a.s.

(iii) $\bar{X}=\bar{X}_{0}+\bar{B}+\bar{M}^{c}+\bar{M}^{d}+\bar{J} \overline{\mathbb{P}}_{0}$-a.s.

Proof. By the Skorokhod representation theorem, both sequences $\left(z^{n, \bar{X}}\right)$ and $\left(z^{n, \bar{J}}\right)$ converge pointwise to $z^{0, \bar{X}}$ and $z^{0, \bar{J}}$, respectively, on the Skorokhod space. Now, as $x-h(x)$ is a continuous function vanishing in a neighborhood of zero, the function $I^{x-h(x)}: \Omega \rightarrow \Omega$ defined in (12) is continuous; hence

$$
\lim _{n \rightarrow \infty} I^{x-h(x)}\left(z^{n, \bar{X}}\right)=I^{x-h(x)}\left(z^{0, \bar{X}}\right) .
$$


On the other hand, as $\bar{J}=\sum_{0 \leq s \leq .}\left[\Delta \bar{X}_{s}-h\left(\Delta \bar{X}_{s}\right)\right] \overline{\mathbb{P}}_{n}$-a.s. for each $n$, we deduce from the relation $\overline{\mathbb{P}}_{n}=\lambda \circ\left(z^{n}\right)^{-1}$ that

$$
\lambda\left[z^{n, \bar{J}}=I^{x-h(x)}\left(z^{n, \bar{X}}\right)\right]=1 .
$$

Therefore, we conclude that $z^{0, \bar{J}}=I^{x-h(x)}\left(z^{0, \bar{X}}\right) \lambda$-a.s., which in turn implies (i).

Next, as each $g_{i}(x)$ is a continuous function vanishing in a neighborhood of the origin, the same argument as in (i), but with $g_{i}(x)$ and $\bar{U}^{i}$, yields (ii).

To prove (iii), we notice the existence of a different topology on the Skorokhod space $\Omega$, the so-called $S$-topology, which is weaker than the usual Skorokhod $J_{1^{-}}$ topology, but has the property that the addition $(\alpha, \beta) \in \Omega \times \Omega \mapsto \alpha+\beta \in \Omega$ is sequentially continuous; see [23, Theorem 2.13]. Therefore, we conclude that

$$
\begin{aligned}
& \lim _{n \rightarrow \infty}\left(z^{n, \bar{X}}-z_{0}^{n, \bar{X}}-z^{n, \bar{B}}-z^{n, \bar{M}^{c}}-z^{n, \bar{M}^{d}}-z^{n, \bar{J}}\right) \\
= & z^{0, \bar{X}}-z_{0}^{0, \bar{X}}-z^{0, \bar{B}}-z^{0, \bar{M}^{c}}-z^{0, \bar{M}^{d}}-z^{0, \bar{J}}
\end{aligned}
$$

pointwise in the $S$-topology. On the other hand, $\bar{X}=\bar{X}_{0}+\bar{B}+\bar{M}^{c}+\bar{M}^{d}+\bar{J} \overline{\mathbb{P}}_{n}$-a.s.; hence $z^{n, \bar{X}}-z_{0}^{n, \bar{X}}-z^{n, \bar{B}}-z^{n, \bar{M}^{c}}-z^{n, \bar{M}^{d}}-z^{n, \bar{J}}=0 \lambda$-a.s. for each $n$. Therefore, we conclude that also $z^{0, \bar{X}}-z_{0}^{0, \bar{X}}-z^{0, \bar{B}}-z^{0, \bar{M}^{c}}-z^{0, \bar{M}^{d}}-z^{0, \bar{J}}=0 \lambda$-a.s., which indeed gives us (iii).

For any constant $K>0$, we denote by $\operatorname{Lip}_{K}$ the set of all Lipschitz-continuous functions on $[0, T]$ with Lipschitz constant $K$. Recall that Condition (B) provides the finiteness of

$$
\mathcal{K}:=\sup _{(b, c, F) \in \Theta}\left\{|b|+|c|+\int_{\mathbb{R}^{d}}|x|^{2} \wedge|x| F(d x)\right\}<\infty .
$$

Lemma 3.6. Let $\Theta \subseteq \mathbb{R}^{d} \times \mathbb{S}_{+}^{d} \times \mathcal{L}$ satisfy Condition (B) and let $\left(\overline{\mathbb{P}}_{n}\right)_{n \in \mathbb{N}} \subseteq$ $\overline{\mathfrak{P}}_{\text {sem }}^{a c}(\Theta)$ be a sequence converging weakly to some law $\overline{\mathbb{P}}_{0} \in \mathfrak{M}_{1}(\bar{\Omega})$. Then $\bar{B}$ is absolutely continuous $\overline{\mathbb{P}}_{0}$-a.s. satisfying $\mathbb{E}^{\overline{\mathbb{P}}_{0}}\left[\int_{0}^{T}\left|\bar{b}_{s}\right| d s\right]<\infty$. In particular, it is $\overline{\mathbb{F}}$-predictable of $\overline{\mathbb{P}}_{0}$-integrable variation. Moreover, the same holds true for $\bar{C}$ and $\widetilde{\widetilde{C}}$.

Proof. As $\Theta \subseteq \mathbb{R}^{d} \times \mathbb{S}_{+}^{d} \times \mathcal{L}$ satisfy Condition (B), we have $\overline{\mathbb{P}}_{n}$-a.s. that for any $0 \leq s \leq t \leq T$,

$$
\left|\bar{B}_{t}-\bar{B}_{s}\right| \leq \int_{s}^{t}\left|\bar{b}_{r}\right| d r \leq \mathcal{K}|t-s|
$$

hence $\bar{B} \in \operatorname{Lip}_{\mathcal{K}} \overline{\mathbb{P}}_{n}$-a.s. We can conclude by

$$
\begin{aligned}
1 & =\overline{\mathbb{P}}_{n}\left[\left|\bar{B}_{t}-\bar{B}_{s}\right| \leq \mathcal{K}|t-s|, \forall t, s \in[0, T] \cap \mathbb{Q}\right] \\
& =\lambda\left[\left|z_{t}^{n, \bar{B}}-z_{s}^{n, \bar{B}}\right| \leq \mathcal{K}|t-s|, \forall t, s \in[0, T] \cap \mathbb{Q}\right]
\end{aligned}
$$

that $z^{n, \bar{B}} \in \operatorname{Lip}_{\mathcal{K}}, \lambda$-a.s. for each $n$. As $z^{n, \bar{B}}$ converges to $z^{0, \bar{B}}$ pointwise in $\Omega_{d}^{c}$, we have $z^{0, \bar{B}} \in \operatorname{Lip}_{\mathcal{K}} \lambda$-a.s., which means that $\bar{B} \in \operatorname{Lip}_{\mathcal{K}} \overline{\mathbb{P}}_{0}$-a.s. Hence, by Rademacher's theorem, $\bar{B}$ has $\overline{\mathbb{P}}_{0}$-a.s. absolutely continuous trajectories, and $\mathbb{E}^{\overline{\mathbb{P}}_{0}}\left[\int_{0}^{T}\left|\bar{b}_{s}\right| d s\right] \leq \mathcal{K} T$. The finite variation property of $\bar{B}$ follows. Note that by continuity, $\bar{B}$ is $\overline{\mathbb{F}}$-predictable. 
Next, the same arguments as above yield also the $\overline{\mathbb{P}}_{0}$-a.s. absolute continuity of $\bar{C}$ with the same integrability property. Moreover, as by [21, II.2.18, p. 79], each component satisfies

$$
\overline{\widetilde{C}}^{i j}=\bar{C}^{i j}+\int_{0} \int_{\mathbb{R}^{d}} h^{i}(x) h^{j}(x) \bar{F}_{t}^{\overline{\mathbb{P}}_{n}}(d x) d t \quad \overline{\mathbb{P}}_{n} \text {-a.s. } \quad \text { for each } n,
$$

the same also holds for $\overline{\widetilde{C}}$.

Lemma 3.7. Let $\Theta \subseteq \mathbb{R}^{d} \times \mathbb{S}_{+}^{d} \times \mathcal{L}$ satisfy Condition $(\mathrm{B})$ and let $\left(\overline{\mathbb{P}}_{n}\right)_{n \in \mathbb{N}} \subseteq$ $\overline{\mathfrak{P}}_{\text {sem }}^{a c}(\Theta)$ be a sequence converging weakly to some law $\overline{\mathbb{P}}_{0} \in \mathfrak{M}_{1}(\bar{\Omega})$. Then:

(i) both $\bar{M}^{c}$ and $\bar{M}^{d}$ are $\overline{\mathbb{P}}_{0}-\overline{\mathbb{F}}$-martingales.

(ii) For each $i \in \mathbb{N}$, we have $\bar{V}^{i}=g_{i}(x) * \nu^{\overline{\mathbb{P}}_{0}} \quad \overline{\mathbb{P}}_{0}$-a.s.

Proof. To see that $\bar{M}^{d}$ is a $\overline{\mathbb{P}}_{0}-\overline{\mathbb{F}}$-martingale we first claim that for each $t \in[0, T]$, the sequence $\left(\bar{M}_{t}^{d} \mid \overline{\mathbb{P}}_{n}\right)$ is uniformly integrable in the sense that

$$
\lim _{a \rightarrow \infty} \sup _{n \in \mathbb{N}} \mathbb{E}^{\overline{\mathbb{P}}_{n}}\left[\bar{M}_{t}^{d} \mathbb{1}_{\left\{\left|\bar{M}_{t}^{d}\right| \geq a\right\}}\right]=0 .
$$

To see this, observe that due to $\Theta$ satisfying Condition (B),

$$
\mathbb{E}^{\overline{\mathbb{P}}_{n}}\left[\left|\bar{M}_{t}^{d}\right|^{2}\right]=\mathbb{E}^{\overline{\mathbb{P}}_{n}}\left[\left|\left[\bar{M}^{d}\right]_{t}\right|\right] \leq \mathbb{E}^{\overline{\mathbb{P}}_{n}}\left[\int_{0}^{t} \int_{\mathbb{R}^{d}}|h(x)|^{2} \bar{F}_{s}^{\overline{\mathbb{P}}_{n}}(d x) d s\right] \leq K \mathcal{K} T
$$

uniformly for all $n$; hence the result follows from the de la Vallée-Poussin theorem. Then in terms of the Skorokhod representation, this means that for all $t \in[0, T]$, the sequence of random variables $\left(z_{t}^{n, \bar{M}^{d}}\right)_{n \in \mathbb{N}}$ defined on $([0,1], \mathcal{B}([0,1]), \lambda)$ is uniformly integrable.

Next, note that the canonical process $\overline{\mathbb{X}}$ on $\bar{\Omega}$ with state space $S:=\mathbb{R}^{5 d+3 d^{2}} \times$ $\left(\mathbb{R}^{2}\right)^{\mathbb{N}}$ is right-continuous and the set $\mathfrak{J}:=\left\{t \in[0, T] \mid \overline{\mathbb{P}}_{0}\left[\Delta \overline{\mathbb{X}}_{t} \neq 0\right]>0\right\}$ is at most countable. Thus, the set $\mathfrak{D}:=[0, T] \backslash \mathfrak{J}$ is dense in $[0, T]$, and for all $t \in \mathfrak{D}$ the sequence of laws $\left(\overline{\mathbb{P}}_{n} \circ \overline{\mathbb{X}}_{t}^{-1}\right)_{n \in \mathbb{N}}$ converges weakly to $\overline{\mathbb{P}}_{0} \circ \overline{\mathbb{X}}_{t}^{-1}$. In terms of the Skorokhod representation, the latter means that for all $t \in \mathfrak{D}, \lim _{n \rightarrow \infty} z_{t}^{n}=z_{t}^{0}$ in $S \lambda$-a.s. Thus, for any $s, t \in \mathfrak{D}$ with $s<t$, for any finite partition $0 \leq s_{1}<\cdots<$ $s_{m} \leq s$ taking values in $\mathfrak{D}$, and for any $f_{j} \in C_{b}(S), j=1, \ldots, m$, we have by the uniform integrability of the sequence $\left(z_{t}^{n, \bar{M}^{d}}-z_{s}^{n, \bar{M}^{d}}\right)_{n \in \mathbb{N}}$ that

$$
\begin{aligned}
0 & =\lim _{n \rightarrow \infty} \mathbb{E}^{\overline{\mathbb{P}}_{n}}\left[\prod_{j=1}^{m} f_{j}\left(\overline{\mathbb{X}}_{s_{j}}\right)\left(\bar{M}_{t}^{d}-\bar{M}_{s}^{d}\right)\right] \\
& =\lim _{n \rightarrow \infty} \mathbb{E}^{\lambda}\left[\prod_{j=1}^{m} f_{j}\left(z_{s_{j}}^{n}\right)\left(z_{t}^{n, \bar{M}^{d}}-z_{s}^{n, \bar{M}^{d}}\right)\right] \\
& =\mathbb{E}^{\lambda}\left[\prod_{j=1}^{m} f_{j}\left(z_{s_{j}}^{0}\right)\left(z_{t}^{0, \bar{M}^{d}}-z_{s}^{0, \bar{M}^{d}}\right)\right] \\
& =\mathbb{E}^{\overline{\mathbb{P}}_{0}}\left[\prod_{j=1}^{m} f_{j}\left(\overline{\mathbb{X}}_{s_{j}}\right)\left(\bar{M}_{t}^{d}-\bar{M}_{s}^{d}\right)\right] .
\end{aligned}
$$

As the filtration $\overline{\mathbb{F}}$ is generated by the canonical process $\left(\overline{\mathbb{X}}_{t}\right)_{t \in[0, T]}$, we can deduce from the monotone class theorem that $\left(\bar{M}_{t}^{d}\right)_{t \in \mathfrak{D}}$ is an $\overline{\mathbb{F}}$-martingale under $\overline{\mathbb{P}}_{0}$, and 
as $\mathfrak{D}$ is dense in $[0, T]$, the whole process $\left(\bar{M}_{t}^{d}\right)_{t \in[0, T]}$ is also an $\overline{\mathbb{F}}$-martingale under $\overline{\mathbb{P}}_{0}$. Finally, we observe that the same argument also works for $\bar{M}^{c}$; hence (i) holds.

Now, fix any $i \in \mathbb{N}$. By continuity, $\bar{V}^{i}$ is $\mathbb{F}$-predictable. Moreover, as $g_{i}$ is a bounded continuous function vanishing in a neighborhood of the origin, there exists $0<\delta_{i} \leq 1$ such that for each $n$,

$$
\bar{V}^{i}=\int_{0}^{\cdot} \int_{\mathbb{R}^{d}} g_{i}(x) \bar{F}_{s}^{\overline{\mathbb{P}}_{n}}(d x) d s=\int_{0}^{\cdot} \int_{\left\{|x| \geq \delta_{i}\right\}} g_{i}(x) \bar{F}_{s}^{\overline{\mathbb{P}}_{n}}(d x) d s \quad \overline{\mathbb{P}}_{n^{-}} \text {-a.s. }
$$

Thus, we can argue as in Lemma 3.6 to conclude that for $c_{i}:=\sup _{x}\left|g_{i}(x)\right|$ and $\widetilde{\mathcal{K}}_{i}:=c_{i} \mathcal{K} / \delta_{i}^{2}$, we have $\bar{V}^{i} \in \operatorname{Lip}_{\widetilde{\mathcal{K}}_{i}} \overline{\mathbb{P}}_{n}$-a.s. for all $n$ and then also $\bar{V}^{i} \in \operatorname{Lip}_{\widetilde{\mathcal{K}}_{i}} \overline{\mathbb{P}}_{0^{-}}$-a.s. Therefore, by applying Lemma 3.5(ii), it remains to show that $\bar{W}^{i}:=\bar{U}^{i}-\bar{V}^{i}$ is a $\overline{\mathbb{P}}_{0}-\overline{\mathbb{F}}$-(local)-martingale. But this follows directly when applying the same arguments as in (i), but with respect to $\bar{W}^{i}$.

Remark 3.8. Whereas $\bar{M}^{c}$ is a continuous $\overline{\mathbb{F}}$-martingale under $\overline{\mathbb{P}}_{0}$, it is not necessarily true that $\bar{M}^{d}$ is a purely discontinuous $\overline{\mathbb{P}}_{0}-\overline{\mathbb{F}}$-martingale. We recall that our goal in this section is to provide a necessary and sufficient condition for $\bar{M}^{d}$ to be a purely discontinuous martingale under $\overline{\mathbb{P}}_{0}$; see Proposition 3.1

Lemma 3.9. Let $\Theta \subseteq \mathbb{R}^{d} \times \mathbb{S}_{+}^{d} \times \mathcal{L}$ satisfy Condition $(\mathrm{B})$ and let $\left(\overline{\mathbb{P}}_{n}\right)_{n \in \mathbb{N}} \subseteq$ $\overline{\mathfrak{P}}_{\text {sem }}^{a c}(\Theta)$ be a sequence converging weakly to some law $\overline{\mathbb{P}}_{0} \in \mathfrak{M}_{1}(\bar{\Omega})$. Then the $\overline{\mathbb{P}}_{0}-\overline{\mathbb{F}}$-compensator $\nu^{\overline{\mathbb{P}}_{0}}(d x, d t)$ of the measure $\mu^{\bar{X}}(d x, d t)$ associated to the jumps of $\bar{X}$ is absolutely continuous in the sense that $\nu^{\overline{\mathbb{P}}_{0}}(d x, d t)$ satisfies a disintegration $\nu^{\overline{\mathbb{P}}_{0}}(d x, d t)=\bar{F}_{t}^{\overline{\mathbb{P}}_{0}}(d x) d t \overline{\mathbb{P}}_{0}$-a.s.

Proof. A careful inspection of the proof of [21, Proposition II.2.9, p. 77] shows that the absolute continuity of $\nu^{\overline{\mathbb{P}}_{0}}$ is equivalent to the absolute continuity of the process $\bar{A}:=\left(1 \wedge|x|^{2}\right) * \nu^{\overline{\mathbb{P}}_{0}}$.

Recall the countable family $\mathcal{C}^{+}\left(\mathbb{R}^{d}\right):=\left\{g_{i} \mid i \in \mathbb{N}\right\}$ introduced at the beginning of Subsection 3.1. In particular, for each $m \in \mathbb{N}, g_{2 m}$ is a continuous function on $\mathbb{R}^{d}$ satisfying for all $x \in \mathbb{R}^{d}$ that $0 \leq g_{2 m}(x) \leq|x|^{2} \wedge 1$ and that

$$
g_{2 m}(x)= \begin{cases}0 & \text { if }|x| \leq \frac{1}{2 m}, \\ |x|^{2} \wedge 1 & \text { if }|x|>\frac{1}{m} .\end{cases}
$$

Then by definition, we have for each $n$ that

$$
\bar{V}^{2 m}=\int_{0}^{\cdot} \int_{\mathbb{R}^{d}} g_{2 m}(x) \bar{F}_{s}^{\overline{\mathbb{P}}_{n}}(d x) d s \quad \overline{\mathbb{P}}_{n^{-} \text {-a.s. }}
$$

Thus for each $m$, by the same argument as in Lemma 3.7(ii) we obtain that $\bar{V}^{2 m} \in$ $\operatorname{Lip}_{\mathcal{K}} \overline{\mathbb{P}}_{0}$-a.s. Therefore, Fatou's lemma and Lemma 3.7(ii) yield

$$
\mathbb{E}^{\overline{\mathbb{P}}_{0}}\left[\bar{A}_{T}\right] \leq \liminf _{m \rightarrow \infty} \mathbb{E}^{\overline{\mathbb{P}}_{0}}\left[\bar{V}_{T}^{2 m}\right] \leq \mathcal{K} T ;
$$

in particular, $\bar{A}_{t}<\infty \overline{\mathbb{P}}_{0}$-a.s. for all $t \in[0, T]$. Thus, by dominated convergence, $\bar{A}_{t}=\lim _{m \rightarrow \infty} \bar{V}_{t}^{2 m} \overline{\mathbb{P}}_{0}$-a.s. for each $t \in[0, T]$; hence also

$$
\bar{A}=\sup _{m \in \mathbb{N}} \bar{V}^{2 m} \overline{\mathbb{P}}_{0} \text {-a.s. }
$$


As $\left(\bar{V}^{2 m}\right)_{m \in \mathbb{N}} \subseteq \operatorname{Lip}_{\mathcal{K}}$, we obtain that $\bar{A} \in \operatorname{Lip}_{\mathcal{K}}$, which implies its absolute continuity $\overline{\mathbb{P}}_{0}$-a.s.

Lemma 3.10. Let $\Theta \subseteq \mathbb{R}^{d} \times \mathbb{S}_{+}^{d} \times \mathcal{L}$ satisfy Condition $(\mathrm{B})$ and let $\left(\overline{\mathbb{P}}_{n}\right)_{n \in \mathbb{N}} \subseteq$ $\overline{\mathfrak{P}}_{\text {sem }}^{\text {ac }}(\Theta)$ be a sequence converging weakly to some law $\overline{\mathbb{P}}_{0} \in \mathfrak{M}_{1}(\bar{\Omega})$. Then, the following hold true:

(i) $\bar{C}$ is the quadratic covariation of $\bar{M}^{c}$ under $\overline{\mathbb{P}}_{0}-\overline{\mathbb{F}}$.

(ii) $\overline{[M]}$ is the quadratic covariation of $\bar{M}:=\bar{M}^{c}+\bar{M}^{d}$ under $\overline{\mathbb{P}}_{0}-\overline{\mathbb{F}}$.

(iii) $\overline{\widetilde{C}}$ is the modified second characteristic of $\bar{X}$ under $\overline{\mathbb{P}}_{0}-\overline{\mathbb{F}}$.

Proof. To obtain (i), denote by $\left\langle\bar{M}^{c}\right\rangle^{\overline{\mathbb{P}}_{0}}$ the quadratic variation of $\bar{M}^{c}$ under $\overline{\mathbb{P}}_{0}-\overline{\mathbb{F}}$. As $\bar{C}$ is the quadratic variation of $\bar{M}^{c}$ under each $\overline{\mathbb{P}}_{n}$, we deduce from [21, Corollary VI.6.29, p. 385] that $\left(\bar{M}^{c}, \bar{C}\right)=\left(\bar{M}^{c},\left\langle\bar{M}^{c}\right\rangle^{\mathbb{P}_{0}}\right)$ in law under $\overline{\mathbb{P}}_{0}$. This implies that also $\left(\bar{M}^{c}, \bar{M}^{c}, \bar{C}\right)=\left(\bar{M}^{c}, \bar{M}^{c},\left\langle\bar{M}^{c}\right\rangle^{\mathbb{P}_{0}}\right)$ in law under $\overline{\mathbb{P}}_{0}$. By applying 21, Theorem VI.6.22, p. 383], we see that $\left(\bar{M}^{c}, \bar{M}^{c}, \bar{C}, \int \bar{M}^{c} d \bar{M}^{c}\right)=\left(\bar{M}^{c}, \bar{M}^{c},\left\langle\bar{M}^{c}\right\rangle^{\mathbb{P}_{0}}\right.$, $\int \bar{M}^{c} d \bar{M}^{c}$ ) in law under $\overline{\mathbb{P}}_{0}$. As a consequence, we obtain componentwise for any $1 \leq i, j \leq d$ that

$$
\begin{aligned}
& \bar{C}^{i j}-\bar{M}^{c, i} \bar{M}^{c, j}-\int \bar{M}^{c, i} d \bar{M}^{c, j}-\int \bar{M}^{c, j} d \bar{M}^{c, i} \\
= & \left\langle\bar{M}^{c}\right\rangle^{\mathbb{P}_{0}, i j}-\bar{M}^{c, i} \bar{M}^{c, j}-\int \bar{M}^{c, i} d \bar{M}^{c, j}-\int \bar{M}^{c, j} d \bar{M}^{c, i} \\
= & 0
\end{aligned}
$$

in law under $\overline{\mathbb{P}}_{0}$; hence by definition of the quadratic variation, $\bar{C}$ coincides with $\left\langle\bar{M}^{c}\right\rangle^{\bar{P}_{0}}$.

The proof of (ii) is identical to the one for (i); hence it remains to prove (iii).

To obtain (iii), we know from Lemma 3.6 that $\bar{C}$ is of integrable variation under $\overline{\mathbb{P}}_{0}$. Moreover, we know from (ii) that $\overline{[M]}$ is the quadratic variation of $M:=$ $\bar{M}^{c}+\bar{M}^{d}$ under $\overline{\mathbb{P}}_{0}$. Hence by definition of the modified second characteristic, it remains to show that $\overline{[M]}-\overline{\widetilde{C}}$ is a $\overline{\mathbb{P}}_{0}-\overline{\mathbb{F}}$-martingale, which we obtain by applying the same arguments as in Lemma 3.7 once we derive that the sequence $\left(\overline{[M]}_{t}-\right.$ $\left.\widetilde{\widetilde{C}}_{t} \mid \overline{\mathbb{P}}_{n}\right)$ is uniformly integrable for each $t \in[0, T]$. To see this, observe that due to Condition (B),

$$
\begin{aligned}
\mathbb{E}^{\overline{\mathbb{P}}_{n}}\left[\left|\overline{[M]_{t}}-\overline{\widetilde{C}}_{t}\right|^{2}\right]=\mathbb{E}^{\overline{\mathbb{P}}_{n}}\left[\left|[\overline{[M]}-\overline{\widetilde{C}}]_{t}\right|\right] & \leq \mathbb{E}^{\overline{\mathbb{P}}_{n}}\left[\sum_{0 \leq s \leq t}\left|\Delta \overline{[M]_{s}}\right|^{2}\right] \\
& =\mathbb{E}^{\overline{\mathbb{P}}_{n}}\left[\int_{0}^{t} \int_{\mathbb{R}^{d}}|h(x)|^{4} \bar{F}_{s}^{\overline{\mathbb{P}}_{n}}(d x) d s\right] \\
& \leq K \mathcal{K} t
\end{aligned}
$$

uniformly for all $n$, which implies the desired uniform integrability.

Proof of Proposition 3.4. This is simply the summary of Lemmas 3.5 3.7 and Lemmas 3.9 and 3.10, noticing that the absolute continuity of the second characteristic $\bar{C}^{\overline{\mathbb{P}}_{0}}$ (which is not necessarily equal to $\bar{C}$ ) ) of $\bar{X}$ under $\overline{\mathbb{P}}_{0^{-}} \overline{\mathbb{F}}$ follows from the absolute continuity of the modified second characteristic $\bar{C}$ and the third characteristic. 
3.3. Proofs of Proposition 3.1 and Corollary 3.2, The goal of this subsection is to prove Proposition 3.1 and Corollary [3.2. We divide the proofs into several lemmas. To keep the notation short, we provide the proof of Proposition 3.1 in the one-dimensional case, i.e., $\Omega=\mathbb{D}([0, T], \mathbb{R})$. The extension to the multi-dimensional case is straightforward, which we explain right after the proof in Remark 3.15 .

Lemma 3.11. Let $\Theta \subseteq \mathbb{R}^{d} \times \mathbb{S}_{+}^{d} \times \mathcal{L}$ satisfy Condition $(\mathrm{B})$ and let $\left(\overline{\mathbb{P}}_{n}\right)_{n \in \mathbb{N}} \subseteq$ $\overline{\mathfrak{P}}_{\text {sem }}^{a c}(\Theta)$ be a sequence converging weakly to some law $\overline{\mathbb{P}}_{0} \in \mathfrak{M}_{1}(\bar{\Omega})$. Then, the sequence $\overline{\mathbb{P}}_{n} \circ\left(\bar{X}, \bar{M}^{d},\left[\bar{M}^{d}\right]\right)^{-1}$ converges weakly to $\overline{\mathbb{P}}_{0} \circ\left(\bar{X}, \bar{M}^{d},\left[\bar{M}^{d}\right]\right)^{-1}$.

Proof. First, recall that by Proposition 3.4 we know that $\bar{M}^{d}$ is a $\overline{\mathbb{P}}_{0}-\overline{\mathbb{F}}$-martingale; hence $\left[\bar{M}^{d}\right]$ is well-defined also under the limit law $\overline{\mathbb{P}}_{0}$. Moreover, by [21, Corollary VI.6.29, p. 385], the sequence $\overline{\mathbb{P}}_{n} \circ\left(\bar{M}^{d},\left[\bar{M}^{d}\right]\right)^{-1}$ converges weakly to the law $\overline{\mathbb{P}}_{0} \circ$ $\left(\bar{M}^{d},\left[\bar{M}^{d}\right]\right)^{-1}$. From the Skorokhod representation theorem, we obtain $\mathbb{D}\left([0,1], \mathbb{R}^{2}\right)$ valued random variables $\left(\left(z^{n, \bar{M}^{d}}, y^{n}\right)\right)_{n \in \mathbb{N}},\left(z^{0, \bar{M}^{d}}, y^{0}\right)$ on $([0,1], \mathcal{B}([0,1]), \lambda)$ such that $\left(z^{n, \bar{M}^{d}}, y^{n}\right)$ converges to $\left(z^{0, \bar{M}^{d}}, y^{0}\right)$ in $\mathbb{D}\left([0,1], \mathbb{R}^{2}\right)$ pointwisely and for each $n \geq 0$,

$$
\lambda \circ\left(z^{n, \bar{M}^{d}}, y^{n}\right)^{-1}=\overline{\mathbb{P}}_{n} \circ\left(\bar{M}^{d},\left[\bar{M}^{d}\right]\right)^{-1} .
$$

Now, as $\left(z^{n, \bar{X}}\right)$ converges pointwise to $z^{0, \bar{X}}$, there is for each $t$ a sequence $\left(t_{n}\right)$ converging to $t$ such that $\Delta z_{t_{n}}^{n, \bar{X}}$ converges to $\Delta z_{t}^{0, \bar{X}}$; see [21, Proposition VI.2.1, p. 337]. By continuity of the truncation function $h(\cdot)$, also $\left(h\left(\Delta z_{t_{n}}^{n, \bar{X}}\right), h^{2}\left(\Delta z_{t_{n}}^{n, \bar{X}}\right)\right)$ converges to $\left(h\left(\Delta z_{t}^{0, \bar{X}}\right), h^{2}\left(\Delta z_{t}^{0, \bar{X}}\right)\right)$. Moreover, $\left(h\left(\Delta \bar{X}_{t_{n}}\right), h^{2}\left(\Delta \bar{X}_{t_{n}}\right)\right)=\left(\Delta \bar{M}_{t_{n}}^{d n}, \Delta\left[\bar{M}^{d}\right]_{t_{n}}\right) \overline{\mathbb{P}}_{n^{-}}$ a.s., so we have $\left(h\left(\Delta z_{t_{n}}^{n, \bar{X}}\right), h^{2}\left(\Delta z_{t_{n}}^{n, \bar{X}}\right)\right)=\left(\Delta z_{t_{n}}^{n, \bar{M}^{d}}, \Delta y_{t_{n}}^{n}\right) \lambda$-a.s. for each $n$, and from Proposition 3.4 , we know that $\left(h\left(\Delta \bar{X}_{t}\right), h^{2}\left(\Delta \bar{X}_{t}\right)\right)=\left(\Delta \bar{M}_{t}^{d}, \Delta\left[\bar{M}^{d}\right]_{t}\right) \overline{\mathbb{P}}_{0^{-}}$ a.s.; hence $\left(h\left(\Delta z_{t}^{0, \bar{X}}\right), h^{2}\left(\Delta z_{t}^{0, \bar{X}}\right)\right)=\left(\Delta z_{t}^{0, \bar{M}^{d}}, \Delta y_{t}^{0}\right)$. Therefore, we obtain that $\left(\Delta z_{t_{n}}^{n, \bar{M}^{d}}, \Delta y_{t_{n}}^{n}\right)$ converges to $\left(\Delta z_{t}^{0, \bar{M}^{d}}, \Delta y_{t}^{0}\right) \lambda$-a.s. We can then conclude by applying [21, Proposition VI.2.2(b), p. 338] that

$$
\left(z^{n, \bar{X}}, z^{n, \bar{M}^{d}}, y^{n}\right) \text { converges to }\left(z^{0, \bar{X}}, z^{0, \bar{M}^{d}}, y^{0}\right) \text { in } \mathbb{D}\left([0, T], \mathbb{R}^{3}\right) \quad \lambda \text {-a.s. }
$$

which implies the desired result.

From the previous lemma, there exists a sequence of $\mathbb{D}([0, T], \mathbb{R})$-valued random variables $y^{n}$ converging pointwise to $y^{0}$, satisfying

$$
\overline{\mathbb{P}}_{n} \circ\left[\bar{M}^{d}\right]^{-1}=\lambda \circ\left(y^{n}\right)^{-1} \text { for each } n \in \mathbb{N}_{0} .
$$

For the rest of this section, we fix for any $\delta>0$ a continuous function $\psi_{\delta}: \mathbb{R} \rightarrow$ $[0,1]$ such that $\left(\psi_{\delta}\right)_{\delta}$ is a nonincreasing sequence of functions when $\delta$ tends to zero satisfying

$$
\psi_{\delta}(x)= \begin{cases}1 & \text { if }|x| \leq \delta / 2 \\ 0 & \text { if }|x|>\delta\end{cases}
$$


Moreover, for each $n \geq 0$, we introduce

$$
\begin{aligned}
\beta^{n, \delta} & :=\sum_{s \leq \cdot}\left(1-\psi_{\delta}\left(\Delta z_{s}^{n, \bar{X}}\right)\right) h^{2}\left(\Delta z_{s}^{n, \bar{X}}\right), \\
\gamma^{n, \delta} & :=\sum_{s \leq .} \psi_{\delta}\left(\Delta z_{s}^{n, \bar{X}}\right) h^{2}\left(\Delta z_{s}^{n, \bar{X}}\right) .
\end{aligned}
$$

Assume that the conditions of Proposition 3.1 hold true. Under each $\overline{\mathbb{P}}_{n}, \bar{M}^{d}$ is the purely discontinuous martingale part in the canonical representation of the $\overline{\mathbb{P}}_{n^{-}}$ $\overline{\mathbb{F}}$-semimartingale $\bar{X}$, satisfying $\Delta \bar{M}_{t}^{d}=h\left(\Delta \bar{X}_{t}\right) \overline{\mathbb{P}}_{n}$-a.s. Hence, using Lemma 3.11 we have for each $n \geq 1$,

$$
y^{n}=\sum_{0 \leq s \leq .} h^{2}\left(\Delta z_{s}^{n, \bar{X}}\right)=\beta^{n, \delta}+\gamma^{n, \delta} \quad \lambda \text {-a.s. }
$$

However, $\bar{M}^{d}$ is not necessarily a purely discontinuous martingale under $\overline{\mathbb{P}}_{0}$. In fact, as by Proposition $3.4 \Delta \bar{M}^{d}=h(\Delta \bar{X}) \overline{\mathbb{P}}_{0}$-a.s., $\bar{M}^{d}$ is a purely discontinuous martingale if and only if equation (13) also holds true for $n=0$.

Lemma 3.12. For any $\delta>0$, we have:

(i) $\beta^{n, \delta}$ converges pointwise to $\beta^{0, \delta}$ in $\mathbb{D}([0, T], \mathbb{R})$.

(ii) $y^{n}-\beta^{n, \delta}$ converges pointwise to $y^{0}-\beta^{0, \delta}$ in $\mathbb{D}([0, T], \mathbb{R})$.

Proof. Notice that $\left(1-\psi_{\delta}(x)\right) h^{2}(x)$ is a continuous function vanishing in a neighborhood of the origin; hence by (12), the convergence of $\left(z^{n, \bar{X}}\right)$ to $z^{0, \bar{X}}$ implies (i).

To see (ii), we can use (i) and argue as in Lemma 3.11 to see that for each $t$ there exists a sequence $\left(t_{n}\right)$ converging to $t$ such that $\left(\Delta y_{t_{n}}^{n}\right)$ converges to $\Delta y_{t}^{0}$ and $\left(\Delta \beta_{t_{n}}^{n, \delta}\right)$ converges to $\Delta \beta_{t}^{0, \delta}$. Hence (ii) now follows directly from [21, Proposition VI.2.2(a), p. 338].

From now on, we fix a sequence $\left(\delta_{m}\right) \subseteq(0, \infty)$ which converges to 0 .

Lemma 3.13. The following hold true.

(i) $\lim _{m \rightarrow \infty} \beta^{0, \delta_{m}}=\sum_{0 \leq s \leq \cdot} h^{2}\left(\Delta z_{s}^{0, \bar{X}}\right)$ in $\mathbb{D}([0, T], \mathbb{R})$.

(ii) $y^{0, \text { cont }}:=\lim _{m \rightarrow \infty} y^{0}-\beta^{0, \delta_{m}}=y^{0}-\sum_{0 \leq s \leq .} h^{2}\left(\Delta z_{s}^{0, \bar{X}}\right)$ in $\mathbb{D}([0, T], \mathbb{R})$.

Proof. To obtain (i), as each $\beta^{0, \delta_{m}}$ and $\sum_{0 \leq s \leq .} h^{2}\left(\Delta z_{s}^{0, \bar{X}}\right)$ is a nonnegative, nondecreasing function starting in the origin, it suffices to verify that the conditions of [21, Theorem VI.2.15, p. 342] are satisfied. To that end, we want to show that for $t \in[0, T]$,

$$
\lim _{m \rightarrow \infty} \beta_{t}^{0, \delta_{m}}=\sum_{s \leq t} h^{2}\left(\Delta z_{s}^{0, \bar{X}}\right) \quad \text { and } \quad \lim _{m \rightarrow \infty} \sum_{s \leq t}\left|\Delta \beta_{s}^{0, \delta_{m}}\right|^{2}=\sum_{s \leq t}\left|h\left(\Delta z_{s}^{0, \bar{X}}\right)\right|^{4} .
$$

But this follows directly from the definition by applying the monotone convergence theorem, as $\lim _{\delta \rightarrow 0} \psi_{\delta}(x)=0$ for all nonzero $x$.

For (ii), argue as in Lemma 3.12 and apply [21, Proposition VI.2.2(a), p. 338].

Let us summarize what we have shown so far. By Lemma 3.11 and as $\Delta\left[\bar{M}^{d}\right]=$ $h^{2}(\Delta \bar{X}) \overline{\mathbb{P}}_{0}$-a.s., we get $\Delta y^{0}=h^{2}\left(\Delta z^{0, \bar{X}}\right) \lambda$-a.s. Hence, we conclude that $y^{0, \text { cont }}$ 
has continuous paths. Moreover, $\bar{M}^{d}$ is a purely discontinuous $\overline{\mathbb{P}}_{0}-\overline{\mathbb{F}}$-martingale if and only if $y^{0, \text { cont }}=0 \lambda$-a.s. In fact, decomposing

$$
\bar{M}^{d}=\bar{M}^{d, c}+\bar{M}^{d, d}
$$

into its continuous and purely discontinuous martingale parts under $\overline{\mathbb{P}}_{0}$, we see that $\overline{\mathbb{P}}_{0} \circ\left(\left\langle\bar{M}^{d, c}\right\rangle\right)^{-1}=\lambda \circ\left(y^{0, c o n t}\right)^{-1}$. Being a nonnegative, nondecreasing function starting in zero, we obtain $y^{0, \text { cont }}=0 \lambda$-a.s. if and only if $\mathbb{E}^{\lambda}\left[y_{T}^{0, \text { cont }}\right]=0$. Therefore, it remains to show that

$$
\mathbb{E}^{\lambda}\left[y_{T}^{0, \text { cont }}\right]=0 \Longleftrightarrow \lim _{\delta \downarrow 0} \limsup _{n \rightarrow \infty} \mathbb{E}^{\overline{\mathbb{P}}_{n}}\left[\int_{0}^{T} \int_{\{|x| \leq \delta\}}|x|^{2} \bar{F}_{t}^{\overline{\mathbb{P}}_{n}}(d x) d t\right]=0 .
$$

In the subsequent proofs, $K>0$ will be a constant whose value may change from line to line. Moreover, we recall the constant $\mathcal{K}<\infty$ defined in Condition (B).

Lemma 3.14. For each $m \in \mathbb{N}$, the following holds true.

$$
\begin{aligned}
\mathbb{E}^{\lambda}\left[y_{T}^{0}-\beta_{T}^{0, \delta_{m}}\right] & =\lim _{n \rightarrow \infty} \mathbb{E}^{\lambda}\left[\gamma_{T}^{n, \delta_{m}}\right] \\
& =\lim _{n \rightarrow \infty} \mathbb{E}^{\overline{\mathbb{P}}_{n}}\left[\int_{0}^{T} \int_{\mathbb{R}} \psi_{\delta_{m}}(x) h^{2}(x) \bar{F}_{s}^{\overline{\mathbb{P}}_{n}}(d x) d s\right] .
\end{aligned}
$$

Proof. Fix $m \in \mathbb{N}$. We know from Lemma 3.12 that $\gamma^{n, \delta_{m}}$ converges pointwise to $y^{0}-\beta^{0, \delta_{m}}$ in $\mathbb{D}([0, T], \mathbb{R})$. By Proposition $\left[3.4, \bar{X}\right.$ is a $\overline{\mathbb{P}}_{0}-\overline{\mathbb{F}}$-semimartingale with absolutely continuous characteristics; in particular $\overline{\mathbb{P}}_{0}\left[\Delta \bar{X}_{t} \neq 0\right]=0$ for every $t \in[0, T]$. As a consequence, we can conclude that $\lambda\left[\Delta y_{t}^{0} \neq 0\right]=0$ for all $t \in[0, T]$. Therefore, by [21, VI.2.3, p. 339],

$$
\gamma_{T}^{n, \delta_{m}} \text { converges pointwise to } y_{T}^{0}-\beta_{T}^{0, \delta_{m}} \quad \lambda \text {-a.s. when } n \rightarrow \infty \text {. }
$$

To obtain the first equality, it remains to show that the sequence $\left(\gamma_{T}^{n, \delta_{m}}\right)_{n \in \mathbb{N}}$ is uniformly integrable with respect to $\lambda$. By the de la Vallée-Poussin theorem, it suffices to show the boundedness in $L^{2}(\lambda)$ of that sequence. Define the nondecreasing process

$$
\bar{A}_{t}^{m}:=\sum_{0 \leq s \leq t} \psi_{\delta_{m}}\left(\Delta \bar{X}_{s}\right) h^{2}\left(\Delta \bar{X}_{s}\right), \quad t \in[0, T]
$$

Then, for each $n \in \mathbb{N}$, we have $\overline{\mathbb{P}}_{n} \circ\left(\bar{A}_{T}^{m}\right)^{-1}=\lambda \circ\left(\gamma_{T}^{n, \delta_{m}}\right)^{-1}$; hence we need to check that $\sup _{n \in \mathbb{N}} \mathbb{E}^{\overline{\mathbb{P}}_{n}}\left[\left(\bar{A}_{T}^{m}\right)^{2}\right]<\infty$. To that end, by the product rule, write

$$
\left(\bar{A}_{T}^{m}\right)^{2}=2 \int_{0}^{T} \bar{A}_{s-}^{m} d \bar{A}_{s}^{m}+\left[\bar{A}^{m}\right]_{T} .
$$

Moreover, $\bar{A}^{m}$ has

$$
\bar{A}^{m, \overline{\mathbb{P}}_{n}}:=\int_{0}^{\cdot} \int_{\mathbb{R}} \psi_{\delta_{m}}(x) h^{2}(x) \bar{F}_{s}^{\overline{\mathbb{P}}_{n}}(d x) d s
$$


as its $\overline{\mathbb{P}}_{n}-\overline{\mathbb{F}}$-compensator. As $\Theta$ satisfies Condition (B), we obtain from (3) that

$$
\begin{aligned}
& \mathbb{E}^{\overline{\mathbb{P}}_{n}}\left[\left[\bar{A}^{m}\right]_{T}\right]=\mathbb{E}^{\overline{\mathbb{P}}_{n}}\left[\sum_{0 \leq s \leq T} \psi_{\delta_{m}}^{2}\left(\Delta \bar{X}_{s}\right) h^{4}\left(\Delta \bar{X}_{s}\right)\right] \\
& \leq K \mathbb{E}^{\overline{\mathbb{P}}_{n}}\left[\sum_{0 \leq s \leq T} \psi_{\delta_{m}}\left(\Delta \bar{X}_{s}\right) h^{2}\left(\Delta \bar{X}_{s}\right)\right] \\
& =K \mathbb{E}^{\overline{\mathbb{P}}_{n}}\left[\bar{A}_{T}^{m, \overline{\mathbb{P}}_{n}}\right] \\
& \leq K \mathcal{K} T \text {, }
\end{aligned}
$$

where $K$ is a constant only depending on $h$. Similarly, using [21, Theorem I.3.17(iii), p. 32],

$$
\begin{aligned}
\mathbb{E}^{\overline{\mathbb{P}}_{n}}\left[\int_{0}^{T} \bar{A}_{s-}^{m} d \bar{A}_{s}^{m}\right]=\mathbb{E}^{\overline{\mathbb{P}}_{n}}\left[\int_{0}^{T} \bar{A}_{s-}^{m} d \bar{A}_{s}^{m, \overline{\mathbb{P}}_{n}}\right] & \leq \mathbb{E}^{\overline{\mathbb{P}}_{n}}\left[\bar{A}_{T}^{m} \bar{A}_{T}^{m, \overline{\mathbb{P}}_{n}}\right] \\
& \leq K \mathcal{K} T \mathbb{E}^{\overline{\mathbb{P}}_{n}}\left[\bar{A}_{T}^{m}\right] \\
& \leq(K \mathcal{K} T)^{2}
\end{aligned}
$$

Therefore, we conclude that indeed $\sup _{n \in \mathbb{N}} \mathbb{E}^{\overline{\mathbb{P}}_{n}}\left[\left(\bar{A}_{T}^{m}\right)^{2}\right]<\infty$; hence the first equality holds.

The second equality follows simply from the fact that for each $n \in \mathbb{N}$,

$$
\mathbb{E}^{\lambda}\left[\gamma_{T}^{n, \delta_{m}}\right]=\mathbb{E}^{\overline{\mathbb{P}}_{n}}\left[\bar{A}_{T}^{m}\right]=\mathbb{E}^{\overline{\mathbb{P}}_{n}}\left[\bar{A}_{T}^{m, \overline{\mathbb{P}}_{n}}\right]=\mathbb{E}^{\overline{\mathbb{P}}_{n}}\left[\int_{0}^{T} \int_{\mathbb{R}} \psi_{\delta_{m}}(x) h^{2}(x) \bar{F}_{s}^{\overline{\mathbb{P}}_{n}}(d x) d s\right] .
$$

Now we are able to prove Proposition 3.1 .

Proof of Proposition 3.1. Recall that part (i) was already proven in Proposition 3.4. hence it remains to show part (ii).

To see (ii), assume for the first direction that there is $u>0$ such that

$$
\lim _{\delta \downarrow 0} \limsup _{n \rightarrow \infty} \mathbb{E}^{\overline{\mathbb{P}}_{n}}\left[\int_{0}^{T} \int_{\{|x| \leq \delta\}}|x|^{2} \bar{F}_{t}^{\overline{\mathbb{P}}_{n}}(d x) d t\right] \geq u>0 .
$$

As by definition, $\psi_{\delta_{m}} \geq \mathbb{1}_{\left\{|x| \leq \delta_{m} / 2\right\}}$, we get from Lemma 3.14 that for each big enough $m$,

$$
\mathbb{E}^{\lambda}\left[y_{T}^{0}-\beta_{T}^{0, \delta_{m}}\right] \geq \limsup _{n \rightarrow \infty} \mathbb{E}^{\overline{\mathbb{P}}_{n}}\left[\int_{0}^{T} \int_{\left\{|x| \leq \delta_{m} / 2\right\}}|x|^{2} \bar{F}_{t}^{\overline{\mathbb{P}}_{n}}(d x) d t\right] \geq u .
$$

Now, a similar argument as in Lemma 3.14 yields

$$
\mathbb{E}^{\lambda}\left[\left(y_{T}^{n}\right)^{2}\right] \leq 2(K \mathcal{K} T)^{2}+K \mathcal{K} T
$$

uniformly for each $n$, so the sequence $\left(y_{T}^{n}\right)_{n \in \mathbb{N}}$ is uniformly integrable with respect to $\lambda$. As $y^{n}$ converges pointwisely to $y^{0}$ in $\mathbb{D}([0, T], \mathbb{R})$ and $\lambda\left[\Delta y_{t}^{0} \neq 0\right]=0$, we have $y_{T}^{n}$ converging to $y_{T}^{0} \lambda$-a.s., and by uniform integrability also in $L^{1}(\lambda)$. In particular, we see that $\mathbb{E}^{\lambda}\left[y_{T}^{0}\right]<\infty$. Therefore, by dominated convergence, we can conclude that

$$
\mathbb{E}^{\lambda}\left[y_{T}^{0, \text { cont }}\right]=\lim _{m \rightarrow \infty} \mathbb{E}^{\lambda}\left[y_{T}^{0}-\beta_{T}^{0, \delta_{m}}\right] \geq u
$$

hence by the discussion before (14), $\bar{M}^{d}$ is not a purely discontinuous martingale. 
Conversely, suppose that

$$
\lim _{\delta \downarrow 0} \limsup _{n \rightarrow \infty} \mathbb{E}^{\overline{\mathbb{P}}_{n}}\left[\int_{0}^{T} \int_{\{|x| \leq \delta\}}|x|^{2} \bar{F}_{t}^{\overline{\mathbb{P}}_{n}}(d x) d t\right]=0 .
$$

From the fact that $\psi_{\delta_{m}} \leq \mathbb{1}_{\left\{|x| \leq \delta_{m}\right\}}$ and by Lemma 3.14, we have for each big enough $m$ that

$$
\mathbb{E}^{\lambda}\left[y_{T}^{0}-\beta_{T}^{0, \delta_{m}}\right] \leq \limsup _{n \rightarrow \infty} \mathbb{E}^{\overline{\mathbb{P}}_{n}}\left[\int_{0}^{T} \int_{\left\{|x| \leq \delta_{m}\right\}}|x|^{2} \bar{F}_{s}^{\overline{\mathbb{P}}_{n}}(d x) d s\right] .
$$

Therefore, as in (15), we obtain by dominated convergence and by (16) that

$$
\begin{aligned}
\mathbb{E}^{\lambda}\left[y_{T}^{0, \text { cont }}\right] & =\lim _{m \rightarrow \infty} \mathbb{E}^{\lambda}\left[y_{T}^{0}-\beta_{T}^{0, \delta_{m}}\right] \\
& \leq \lim _{m \rightarrow \infty} \limsup _{n \rightarrow \infty} \mathbb{E}^{\overline{\mathbb{P}}_{n}}\left[\int_{0}^{T} \int_{\left\{|x| \leq \delta_{m}\right\}}|x|^{2} \bar{F}_{s}^{\overline{\mathbb{P}}_{n}}(d x) d s\right] \\
& =0 .
\end{aligned}
$$

Thus, $y^{0, \text { cont }}=0 \lambda$-a.s.; hence by the discussion before (14), we have that $\bar{M}^{d}$ is indeed a purely discontinuous martingale.

Remark 3.15. The proof of Proposition 3.1 was given in dimension $d=1$. However, we see from its proof that it can be easily adapted for the multidimensional case. Indeed, clearly, $\bar{M}^{d}:=\left(\bar{M}^{d, 1}, \ldots, \bar{M}^{d, d}\right)$ is a purely discontinuous martingale if and only if each component $\bar{M}^{d, i}$ is. Therefore, one can apply the above proof for each of its components $\bar{M}^{d, i}$. Then,

$$
\lim _{\delta \downarrow 0} \limsup _{n \rightarrow \infty} \mathbb{E}^{\overline{\mathbb{P}}_{n}}\left[\int_{0}^{T} \int_{\{|x| \leq \delta\}}|x|^{2} \bar{F}_{t}^{\overline{\mathbb{P}}_{n}}(d x) d t\right]=0
$$

implies that each of its components $\bar{M}^{d, i}$ is a purely discontinuous martingale; hence so is $\bar{M}^{d}$. On the other hand, if

$$
\lim _{\delta \downarrow 0} \limsup _{n \rightarrow \infty} \mathbb{E}^{\overline{\mathbb{P}}_{n}}\left[\int_{0}^{T} \int_{\{|x| \leq \delta\}}|x|^{2} \bar{F}_{t}^{\overline{\mathbb{P}}_{n}}(d x) d t\right]>0,
$$

then there exists at least one $j \in\{1, \ldots, d\}$ such that $\bar{M}^{d, j}$ is not a purely discontinuous martingale; hence $\bar{M}^{d}$ is not one either.

In the rest of this subsection, we provide the proof of Corollary 3.2 which we present directly in the multidimensional case.

Proof of Corollary [3.2, By Proposition 3.4, $\overline{\mathbb{P}}_{0} \in \overline{\mathfrak{P}}_{s e m}^{a c, w}$. In particular, $\bar{X}$ has canonical representation

$$
\bar{X}=\bar{X}_{0}+\bar{B}+\bar{M}^{c}+\bar{M}^{d}+\bar{J} \quad \overline{\mathbb{P}}_{0} \text {-a.s. }
$$

Moreover, as $\Theta \subseteq \mathbb{R}^{d} \times \mathbb{S}_{+}^{d} \times \mathcal{L}$ satisfies Condition $(\mathrm{J})$, we deduce from Proposition 3.1 that $\bar{M}^{d}$ is a $\overline{\mathbb{P}}_{0}-\overline{\mathbb{F}}$-purely discontinuous martingale, which implies that $\bar{C}$ is the second characteristic of $\bar{X}$ under $\overline{\mathbb{P}}_{0}-\overline{\mathbb{F}}$ and $\overline{\mathbb{P}}_{0} \in \overline{\mathfrak{P}}_{\text {sem }}^{a c}$.

Now, assume for the rest of the proof that in addition, $\Theta \subseteq \mathbb{R}^{d} \times \mathbb{S}_{+}^{d} \times \mathcal{L}$ is closed, convex. Let $\left(\bar{b}^{\overline{\mathbb{P}}_{0}}, \bar{c}^{\overline{\mathbb{P}}_{0}}, \bar{F}^{\overline{\mathbb{P}}_{0}}\right)$ be the $\overline{\mathbb{P}}_{0}-\overline{\mathbb{F}}$-differential characteristics of $\bar{X}$. It remains 
to show that $\left(\bar{b}^{\overline{\mathbb{P}}_{0}}, \bar{c}^{\overline{\mathbb{P}}_{0}}, \bar{F}^{\overline{\mathbb{P}}_{0}}\right) \in \Theta \overline{\mathbb{P}}_{0} \times d t$-a.s. To that end, let us recall the additive, positive homogeneous bijection $\bar{\varphi}: \Theta \rightarrow \bar{\varphi}(\Theta) \subseteq \mathbb{R}^{d} \times \mathbb{S}_{+}^{d} \times \mathbb{R}^{\mathbb{N}}$ defined in (9). Due to (11) it is equivalent to show that $(\bar{b}, \bar{c}, \bar{v}) \in \operatorname{cl}(\bar{\varphi}(\Theta)) \overline{\mathbb{P}}_{0} \times d t$-a.s. Clearly, $\operatorname{cl}(\bar{\varphi}(\Theta))$ is convex and closed. Therefore, as $\bar{\eta}:=(\bar{b}, \bar{c}, \bar{v}) \in \operatorname{cl}(\bar{\varphi}(\Theta)) \overline{\mathbb{P}}_{n} \times d t$-a.s., we have for each $m$ that $m \int_{t}^{t+\frac{1}{m}} \bar{\eta}_{s} d s \in \operatorname{cl}(\bar{\varphi}(\Theta)) \mathbb{P}_{n} \times d t$-a.s., for all $n \in \mathbb{N}$. In other words, for each $n \in \mathbb{N}$,

$$
\mathbb{E}^{\overline{\mathbb{P}}_{n}}\left[\int_{0}^{T} \mathbb{1}_{\left\{m\left(\bar{B}_{t+\frac{1}{m}}-\bar{B}_{t}, \bar{C}_{t+\frac{1}{m}}-\bar{C}_{t},\left(\bar{V}_{t+\frac{1}{m}}^{i}-\bar{V}_{t}^{i}\right)_{i \in \mathbb{N}}\right) \in \operatorname{cl}(\bar{\varphi}(\Theta))\right\}} d t\right]=T .
$$

In terms of the Skorokhod representation, it means that

$$
\mathbb{E}^{\lambda}\left[\int_{0}^{T} \mathbb{1}_{\left\{m\left(z_{t+\frac{1}{m}}^{n, \bar{B}}-z_{t}^{n, \bar{B}}, z_{t+\frac{1}{m}}^{n, \bar{C}}-z_{t}^{n, \bar{C}},\left(z_{t+\frac{1}{m}}^{n, \bar{V}^{i}}-z_{t}^{n, \bar{V}^{i}}\right)_{i \in \mathbb{N}}\right) \in \operatorname{cl}(\bar{\varphi}(\Theta))\right\}} d t\right]=T .
$$

Now, letting $n$ tend to infinity, by applying the dominated convergence theorem, the closedness of $\operatorname{cl}(\bar{\varphi}(\Theta))$ yields

$$
\mathbb{E}^{\lambda}\left[\int_{0}^{T} \mathbb{1}_{\left\{m\left(z_{t+\frac{1}{m}}^{0, \bar{B}}-z_{t}^{0, \bar{B}}, z_{t+\frac{1}{m}}^{0, \bar{C}}-z_{t}^{0, \bar{C}},\left(z_{t+\frac{1}{m}}^{0, \bar{V}^{i}}-z_{t}^{0, \bar{V}^{i}}\right)_{i \in \mathbb{N}}\right) \in \operatorname{cl}(\bar{\varphi}(\Theta))\right\}} d t\right]=T,
$$

which implies that $m\left(\bar{B}_{t+\frac{1}{m}}-\bar{B}_{t}, \bar{C}_{t+\frac{1}{m}}-\bar{C}_{t},\left(\bar{V}_{t+\frac{1}{m}}^{i}-\bar{V}_{t}^{i}\right)_{i \in \mathbb{N}}\right) \in \operatorname{cl}(\bar{\varphi}(\Theta)), \overline{\mathbb{P}}_{0} \times d t-$ a.e. for all $m \in \mathbb{N}$. Now letting $m$ tend to infinity, we obtain that $\bar{\eta}_{t}=\left(\bar{b}_{t}, \bar{c}_{t}, \bar{v}_{t}\right) \in$ $\operatorname{cl}(\bar{\varphi}(\Theta))$ holds $\overline{\mathbb{P}}_{0} \times d t$-a.e.; hence we are done.

3.4. Tightness of $\overline{\mathfrak{P}}_{\text {sem }}^{a c}(\Theta)\left(\Gamma_{0}\right)$. The goal of this subsection is to prove tightness of $\overline{\mathfrak{P}}_{\text {sem }}^{a c}(\Theta)\left(\Gamma_{0}\right)$. More precisely:

Proposition 3.16. Let $\Theta \subseteq \mathbb{R}^{d} \times \mathbb{S}_{+}^{d} \times \mathcal{L}$ satisfy Condition (B) and let $\Gamma_{0} \subseteq \mathfrak{M}_{1}\left(\mathbb{R}^{d}\right)$ be tight. Then the set $\overline{\mathfrak{P}}_{\text {sem }}^{a c}(\Theta)\left(\Gamma_{0}\right)$ defined as in Subsection 3.1 is tight.

In fact, a careful inspection of the proof will lead to the conclusion that with the same arguments we also obtain the tightness of $\mathfrak{P}_{\text {sem }}^{a c}(\Theta)\left(\Gamma_{0}\right)$ on the original space $\mathfrak{M}_{1}(\Omega)$ (when imposing the same conditions as Proposition 3.16$)$; see Corollary 3.22 ,

We divide the proof of Proposition 3.16 into several lemmas. We will use the notation introduced in Section [3.1. We recall the constant $\mathcal{K}<\infty$ defined in Condition (B).

Lemma 3.17. Let $\Theta \subseteq \mathbb{R}^{d} \times \mathbb{S}_{+}^{d} \times \mathcal{L}$ satisfy Condition $(\mathrm{B})$ and let $\left(\overline{\mathbb{P}}_{n}\right)_{n \in \mathbb{N}} \subseteq$ $\overline{\mathfrak{P}}_{\text {sem }}^{a c}(\Theta)$. Then, the sequences $\left(\overline{\mathbb{P}}_{n} \circ \bar{B}^{-1}\right)_{n \in \mathbb{N}},\left(\overline{\mathbb{P}}_{n} \circ \bar{C}^{-1}\right)_{n \in \mathbb{N}},\left(\overline{\mathbb{P}}_{n} \circ \overline{\widetilde{C}}^{-1}\right)_{n \in \mathbb{N}}$ of laws on $\Omega_{d}^{c}$ and $\Omega_{d^{2}}^{c}$ are tight.

Proof. By definition, we have $\overline{\mathbb{P}}_{n}\left[\bar{B}_{0}=0\right]=1$ for each $n$. Moreover, due to $\Theta$ satisfying Condition (B), we have $\overline{\mathbb{P}}_{n}$-a.s. that for any $0 \leq s \leq t \leq T$,

$$
\left|\bar{B}_{t}-\bar{B}_{s}\right| \leq \int_{s}^{t}\left|\bar{b}_{r}\right| d r \leq \mathcal{K}|t-s|
$$

Consequently, by Markov's inequality, we have for each $\varepsilon>0$ that

$$
\limsup _{\delta \downarrow 0} \overline{\mathbb{P}}_{n \in \mathbb{N}}\left[\sup _{t-s \leq \delta ; 0 \leq s \leq t \leq T}\left|\bar{B}_{t}-\bar{B}_{s}\right| \geq \varepsilon\right]=0 .
$$

By applying [6, Theorem 7.3, p. 82], we obtain the tightness of $\left(\overline{\mathbb{P}}_{n} \circ \bar{B}^{-1}\right)_{n \in \mathbb{N}}$. Replacing $\bar{B}$ by $\bar{C}$ in the above proof, we conclude the tightness also for the sequence 
$\left(\overline{\mathbb{P}}_{n} \circ \bar{C}^{-1}\right)_{n \in \mathbb{N}}$. Moreover, using [21, II.2.18, p. 79], the same arguments yield tightness of $\left(\overline{\mathbb{P}}_{n} \circ \overline{\widetilde{C}}^{-1}\right)_{n \in \mathbb{N}}$.

Lemma 3.18. Let $\Theta \subseteq \mathbb{R}^{d} \times \mathbb{S}_{+}^{d} \times \mathcal{L}$ satisfy Condition (B) and let $\left(\overline{\mathbb{P}}_{n}\right)_{n \in \mathbb{N}} \subseteq$ $\overline{\mathfrak{P}}_{\text {sem }}^{\text {ac }}(\Theta)$. Then, both sequences $\left(\overline{\mathbb{P}}_{n} \circ\left(\bar{M}^{c}\right)^{-1}\right)_{n \in \mathbb{N}}$ and $\left(\overline{\mathbb{P}}_{n} \circ\left(\bar{M}^{d}\right)^{-1}\right)_{n \in \mathbb{N}}$ of laws on $\Omega_{d}^{c}$ and $\Omega$ are tight.

Proof. For each $n \in \mathbb{N}$, we have by definition that both $\bar{M}^{c}$ and $\bar{M}^{d}$ are $\overline{\mathbb{P}}_{n}-\overline{\mathbb{F}}$ (locally) square integrable martingales with $\overline{\mathbb{P}}_{n}\left[\bar{M}_{0}^{c}=0\right]=\overline{\mathbb{P}}_{n}\left[\bar{M}_{0}^{d}=0\right]=1$. By Lemma 3.17 the sequence $\left(\overline{\mathbb{P}}_{n} \circ \bar{C}^{-1}\right)_{n \in \mathbb{N}}$ of laws on $\Omega_{d^{2}}^{c}$ is tight. As by definition, $\left\langle\bar{M}^{c}\right\rangle=\bar{C}$ under each $\overline{\mathbb{P}}_{n}$, we deduce the tightness of $\left(\overline{\mathbb{P}}_{n} \circ\left(\bar{M}^{c}\right)^{-1}\right)_{n \in \mathbb{N}}$ directly from [35, Corollary 3, p. 29].

Now, we define the process

$$
\bar{G}, d \overline{\mathbb{P}}_{n}:=\sum_{j=1}^{d} \int_{0} \int_{\mathbb{R}^{d}}\left|h^{j}(x)\right|^{2} \bar{F}_{t}^{\overline{\mathbb{P}}_{n}}(d x) d t .
$$

Since $\Theta$ satisfies Condition (B), we can apply the same arguments as in Lemma 3.17 to obtain the tightness of the sequence $\left(\overline{\mathbb{P}}_{n} \circ\left(\bar{G}^{d, \overline{\mathbb{P}}_{n}}\right)^{-1}\right)_{n \in \mathbb{N}}$ as laws on $\Omega_{1}^{c}$ or equivalently the $C$-tightness if we think of them as laws on $\mathbb{D}([0, T], \mathbb{R})$. As a consequence, we get the tightness of the sequence of laws $\left(\overline{\mathbb{P}}_{n} \circ\left(\bar{M}^{d}\right)^{-1}\right)_{n \in \mathbb{N}}$ directly from [21, Theorem VI.4.13, p. 358].

For any adapted process $\bar{Z}$ defined on $(\bar{\Omega}, \overline{\mathcal{F}}, \overline{\mathbb{F}})$ and any law $\overline{\mathbb{P}}$ defined on that filtered measurable space, we write $(\bar{Z}, \overline{\mathbb{P}})$ when considering $(\bar{\Omega}, \overline{\mathcal{F}}, \overline{\mathbb{F}}, \overline{\mathbb{P}})$ as its stochastic basis. Moreover, we refer to [21, p. 377] for the standard notion for a sequence of processes to be Predictably Uniformly Tight (P-UT).

Lemma 3.19. Let $\Theta \subseteq \mathbb{R}^{d} \times \mathbb{S}_{+}^{d} \times \mathcal{L}$ satisfy Condition (B) and let $\left(\overline{\mathbb{P}}_{n}\right)_{n \in \mathbb{N}} \subseteq$ $\overline{\mathfrak{P}}_{\text {sem }}^{a c}(\Theta)$. Then, the sequence $\left(\bar{X}, \overline{\mathbb{P}}_{n}\right)_{n \in \mathbb{N}}$ is $(P-U T)$.

Proof. Fix any $t \in[0, T]$ and let $i=1, \ldots, d$. Due to Condition (B), the variation of $B^{i}$ satisfies

$$
\sup _{n} \mathbb{E}^{\overline{\mathbb{P}}_{n}}\left[\operatorname{Var}\left(\bar{B}^{i}\right)_{t}\right] \leq \sup _{n} \mathbb{E}^{\overline{\mathbb{P}}_{n}}\left[\int_{0}^{t}\left|\bar{b}_{s}\right| d s\right] \leq \mathcal{K} t
$$

hence tightness of $\left(\overline{\mathbb{P}}_{n} \circ\left(\operatorname{Var}\left(\bar{B}^{i}\right)_{t}\right)^{-1}\right)_{n \in \mathbb{N}}$ follows directly from the Markov inequality. $d$,

Now, the modified second characteristic $\overline{\widetilde{C}}$ satisfies in each component $1 \leq k, l \leq$

$$
\overline{\widetilde{C}}^{k l}=\bar{C}^{k l}+\int_{0}^{\cdot} \int_{\mathbb{R}^{d}} h^{k}(x) h^{l}(x) \bar{F}_{s}^{\overline{\mathbb{P}}_{n}}(d x) d s \quad \overline{\mathbb{P}}_{n^{-}} \text {-a.s. }
$$

hence we can argue as above to obtain tightness of the sequence $\left(\mathbb{P}_{n} \circ\left(\overline{\widetilde{C}}_{t}^{i i}\right)^{-1}\right)_{n \in \mathbb{N}}$.

Next, we see that for the variation of $\bar{J}$, we have

$$
\begin{aligned}
\mathbb{E}^{\overline{\mathbb{P}}_{n}}\left[\operatorname{Var}(\bar{J})_{t}\right] & =\mathbb{E}^{\overline{\mathbb{P}}_{n}}\left[\int_{0}^{t} \int_{\mathbb{R}^{d}}|x-h(x)| \mu^{\bar{X}}(d x) d s\right] \\
& =\mathbb{E}^{\overline{\mathbb{P}}_{n}}\left[\int_{0}^{t} \int_{\mathbb{R}^{d}}|x-h(x)| \bar{F}_{s}^{\overline{\mathbb{P}}_{n}}(d x) d s\right] .
\end{aligned}
$$


Hence in the same way as above, we get also the tightness of the sequence $\left(\overline{\mathbb{P}}_{n} \circ\right.$ $\left.\operatorname{Var}(\bar{J})_{t}^{-1}\right)_{n \in \mathbb{N}}$. As under each $\overline{\mathbb{P}}_{n}, \bar{X}$ is a $\overline{\mathbb{P}}_{n}-\overline{\mathbb{F}}$-semimartingale with canonical representation (7), we conclude that the sequence $\left(\bar{X}, \overline{\mathbb{P}}_{n}\right)_{n \in \mathbb{N}}$ is (P-UT) directly from [21, Theorem 6.15 , p. 380].

Lemma 3.20. Let $\Theta \subseteq \mathbb{R}^{d} \times \mathbb{S}_{+}^{d} \times \mathcal{L}$ satisfy Condition $(\mathrm{B})$, let $\Gamma_{0} \subseteq \mathfrak{M}_{1}\left(\mathbb{R}^{d}\right)$ be tight, and let $\left(\overline{\mathbb{P}}_{n}\right)_{n \in \mathbb{N}} \subseteq \overline{\mathfrak{P}}_{\text {sem }}^{a c}(\Theta)\left(\Gamma_{0}\right)$. Then, the sequence $\left(\overline{\mathbb{P}}_{n} \circ \bar{X}^{-1}\right)_{n \in \mathbb{N}}$ is tight.

Proof. As $\bar{X}$ is a $\overline{\mathbb{P}}_{n}-\overline{\mathbb{F}}$-semimartingale for each $n$, it suffices to verify that the conditions in [21, Theorem VI.4.18, p. 359] are satisfied.

By assumption, $\left(\overline{\mathbb{P}}_{n} \circ\left(\bar{X}_{0}\right)^{-1}\right)_{n \in \mathbb{N}} \subseteq \Gamma_{0}$ is tight. By Lemma 3.19. $\left(\bar{X}, \overline{\mathbb{P}}_{n}\right)_{n \in \mathbb{N}}$ is P-UT; hence by [21, Theorem 6.16 , p. 380],

$$
\lim _{a \uparrow \infty} \sup _{n \in \mathbb{N}} \overline{\mathbb{P}}_{n}\left[\nu^{\mathbb{P}_{n}}([0, T] \times\{x:|x|>a\})>\varepsilon\right]=0 .
$$

Consider the following increasing process:

$$
\bar{D}^{\overline{\mathbb{P}}_{n}}:=\sum_{i=1}^{d}\left[\operatorname{Var}\left(\bar{B}^{i}\right)+\bar{C}^{i i}\right]+\int_{0} \int_{\mathbb{R}^{d}}|x|^{2} \wedge 1 \bar{F}_{s}^{\overline{\mathbb{P}}_{n}}(d x) d s .
$$

Using the same argument as in Lemma 3.17, we obtain the C-tightness of the sequence $\left(\overline{\mathbb{P}}_{n} \circ\left(\bar{D}^{\overline{\mathbb{P}}_{n}}\right)^{-1}\right)_{n \in \mathbb{N}}$. Therefore, by [21, Remark VI.4.20, pp. 359-360], the conditions of [21, Theorem VI.4.18, p. 359] are satisfied; hence we get the result.

Lemma 3.21. Let $\Theta \subseteq \mathbb{R}^{d} \times \mathbb{S}_{+}^{d} \times \mathcal{L}$ satisfy Condition (B) and let $\left(\overline{\mathbb{P}}_{n}\right)_{n \in \mathbb{N}} \subseteq$ $\overline{\mathfrak{P}}_{\text {sem }}^{a c}(\Theta)$. Then the following hold true.

(i) The sequence of laws $\left(\overline{\mathbb{P}}_{n} \circ \bar{J}^{-1}\right)_{n \in \mathbb{N}}$ on $\Omega$ is tight.

(ii) The sequence of laws $\left(\overline{\mathbb{P}}_{n} \circ\left(\bar{U}^{i}\right)^{-1}\right)_{n \in \mathbb{N}}$ on $\Omega_{1}$ is tight for each $i \in \mathbb{N}$.

(iii) The sequence of laws $\left(\overline{\mathbb{P}}_{n} \circ\left(\bar{V}^{i}\right)^{-1}\right)_{n \in \mathbb{N}}$ on $\Omega_{1}^{c}$ is tight for each $i \in \mathbb{N}$.

(iv) The sequence of laws $\left(\overline{\mathbb{P}}_{n} \circ \overline{[M]}^{-1}\right)_{n \in \mathbb{N}}$ on $\Omega_{d^{2}}$ is tight.

Proof. Using the notation introduced in (12), we have $\bar{J}=I^{x-h(x)}\left(\bar{X}-\bar{X}_{0}\right) \overline{\mathbb{P}}_{n}$-a.s. for each $n$, and for each $i \in \mathbb{N}, \bar{U}^{i}=I^{g_{i}(x)\left(|x|^{2} \wedge 1\right)}\left(\bar{X}-\bar{X}_{0}\right) \overline{\mathbb{P}}_{n}$-a.s. Therefore, (i) and (ii) follow directly from the tightness of $\left(\overline{\mathbb{P}}_{n} \circ\left(\bar{X}-\bar{X}_{0}\right)^{-1}\right)_{n \in \mathbb{N}}$; see Lemma 3.20, To see that (iii) holds, recall that under each $\overline{\mathbb{P}}_{n}$, we have for each $i \in \mathbb{N}$ that

$$
\bar{V}^{i}=\int_{0} \int_{\mathbb{R}^{d}} g_{i}(x) \bar{F}_{s}^{\overline{\mathbb{P}}_{n}}(d x) d s \quad \overline{\mathbb{P}}_{n^{-} \text {-a.s. }}
$$

As $g_{i}(x)$ is a bounded function vanishing in a neighborhood of the origin, we can argue as in Lemma 3.17 to obtain (iii). Finally, (iv) follows by 35, Theorem II.2, p. 28] from tightness of $\left(\overline{\mathbb{P}}_{n} \circ \overline{\widetilde{C}}^{-1}\right)_{n \in \mathbb{N}}$; see Lemma 3.17

Now we are able to prove Proposition 3.16

Proof of Proposition 3.16. By an application of Prohorov's theorem, it suffices to show that any sequence $\left(\overline{\mathbb{P}}_{n}\right) \subseteq \overline{\mathfrak{P}}_{\text {sem }}^{a c}(\Theta)\left(\Gamma_{0}\right)$ is tight. To that end, fix such a sequence. From all previous lemmas, we have established the tightness of all the 
following families:

$$
\begin{aligned}
& \left(\overline{\mathbb{P}}_{n} \circ \bar{X}^{-1}\right)_{n \in \mathbb{N}}, \quad\left(\overline{\mathbb{P}}_{n} \circ \bar{B}^{-1}\right)_{n \in \mathbb{N}}, \quad\left(\overline{\mathbb{P}}_{n} \circ\left(\bar{M}^{c}\right)^{-1}\right)_{n \in \mathbb{N}}, \quad\left(\overline{\mathbb{P}}_{n} \circ\left(\bar{M}^{d}\right)^{-1}\right)_{n \in \mathbb{N}}, \\
& \left(\overline{\mathbb{P}}_{n} \circ \bar{J}^{-1}\right)_{n \in \mathbb{N}}, \quad\left(\overline{\mathbb{P}}_{n} \circ \bar{C}^{-1}\right)_{n \in \mathbb{N}}, \quad\left(\overline{\mathbb{P}}_{n} \circ \overline{[M]}\right)_{n \in \mathbb{N}}, \quad\left(\overline{\mathbb{P}}_{n} \circ \overline{\widetilde{C}}^{-1}\right)_{n \in \mathbb{N}}, \\
& \left(\overline{\mathbb{P}}_{n} \circ\left(\bar{U}^{i}\right)^{-1}\right)_{n \in \mathbb{N}}, \quad\left(\overline{\mathbb{P}}_{n} \circ\left(\bar{V}^{i}\right)^{-1}\right)_{n \in \mathbb{N}}, \quad i \in \mathbb{N} .
\end{aligned}
$$

Fix $\varepsilon>0$, and let $K(\bar{X}) \subseteq \Omega$ be a compact set such that $\sup _{n \in \mathbb{N}} \overline{\mathbb{P}}_{n}[\bar{X} \notin K(\bar{X})] \leq$ $\frac{\varepsilon}{10}$. In the same way, define the compact sets $K(\bar{B}) \subseteq \Omega_{d}^{c}, \ldots, K(\widetilde{\widetilde{C}}) \subseteq \Omega_{d^{2}}^{c}$. Moreover, choose for each $i \in \mathbb{N}$ compact sets $K\left(\bar{U}^{i}\right) \subseteq \Omega_{1}, K\left(\bar{V}^{i}\right) \subseteq \Omega_{1}^{c}$ such that

$$
\max \left\{\sup _{n \in \mathbb{N}} \overline{\mathbb{P}}_{n}\left[\bar{U}^{i} \notin K\left(\bar{U}^{i}\right)\right], \sup _{n \in \mathbb{N}} \overline{\mathbb{P}}_{n}\left[\bar{V}^{i} \notin K\left(\bar{V}^{i}\right)\right]\right\} \leq \frac{\varepsilon}{10} \frac{1}{2^{i}} .
$$

By Tychonoff's theorem, the set

$$
\bar{K}:=K(\bar{X}) \times K(\bar{B}) \times \cdots \times K(\overline{\widetilde{C}}) \times \Pi_{i \in \mathbb{N}}\left[K\left(\bar{U}^{i}\right) \times K\left(\bar{V}^{i}\right)\right] \subseteq \bar{\Omega}
$$

is compact. Moreover, we have for each $n \in \mathbb{N}$ that

$$
\begin{aligned}
\overline{\mathbb{P}}_{n}\left[\bar{K}^{c}\right] \leq & \overline{\mathbb{P}}_{n}[\bar{X} \notin K(\bar{X})]+\cdots+\overline{\mathbb{P}}_{n}[\overline{\widetilde{C}} \notin K(\overline{\widetilde{C}})] \\
& +\sum_{i=1}^{\infty}\left(\overline{\mathbb{P}}_{n}\left[\bar{U}^{i} \notin K\left(\bar{U}^{i}\right)\right]+\overline{\mathbb{P}}_{n}\left[\bar{V}^{i} \notin K\left(\bar{V}^{i}\right)\right]\right) \\
\leq & \varepsilon .
\end{aligned}
$$

In fact, we observe that by the same arguments as above, we also get tightness of $\mathfrak{P}_{\text {sem }}^{a c}(\Theta)\left(\Gamma_{0}\right)$ in the original space $\mathfrak{M}_{1}(\Omega)$.

Corollary 3.22. Let $\Theta \subseteq \mathbb{R}^{d} \times \mathbb{S}_{+}^{d} \times \mathcal{L}$ satisfy Condition (B) and let $\Gamma_{0} \subseteq \mathfrak{M}_{1}\left(\mathbb{R}^{d}\right)$ be tight. Then $\mathfrak{P}_{\text {sem }}^{a c}(\Theta)\left(\Gamma_{0}\right)$ is tight.

Proof. By an application of Prohorov's theorem, it suffices to show that any sequence $\left(\mathbb{P}_{n}\right) \subseteq \mathfrak{P}_{\text {sem }}^{a c}(\Theta)$ is tight. For any such sequence, we can apply exactly the same argument as in Lemma 3.20 to obtain the tightness result.

\section{Proof of Theorems 2.1, 2.5, and 2.8}

The goal of this section is to prove Theorems 2.1 and 2.5. The strategy of their proofs is the following. In the last section, we stated and proved corresponding results in an enlarged space. We need to find a way how to go back and forth from the original space $\Omega=\mathbb{D}\left([0, T], \mathbb{R}^{d}\right)$ to the enlarged space $\bar{\Omega}$ to conclude the results in the original space $\Omega$. On the one hand, to get from $\Omega$ to $\bar{\Omega}$, we introduce for any measure $\mathbb{P} \in \mathfrak{P}_{\text {sem }}^{a c}(\Theta)$ a corresponding measure $\overline{\mathbb{P}} \in \overline{\mathfrak{P}}_{\text {sem }}^{a c}(\Theta)$ which is simply the probability measure induced by the canonical representation of $X$ under $\mathbb{P}$. On the other hand, to get back from $\bar{\Omega}$ to $\Omega$, we consider for any $\overline{\mathbb{P}} \in \overline{\mathfrak{P}}_{\text {sem }}^{a c}(\Theta)$ the corresponding pushforward measure $\mathbb{P}:=\overline{\mathbb{P}} \circ \bar{X}^{-1}$, which will turn out to be in $\mathfrak{P}_{\text {sem }}^{a c}(\Theta)$.

Let us start with a fixed probability measure $\mathbb{P} \in \mathfrak{P}_{\text {sem }}^{a c}$. Consider the canonical representation of $X$ under $\mathbb{P}-\mathbb{F}_{+}^{\mathbb{P}}$ given by

$$
X=X_{0}+B^{\mathbb{F}_{+}^{\mathbb{P}}}+M^{c, \mathbb{F}_{+}^{\mathbb{P}}}+M^{d, \mathbb{F}_{+}^{\mathbb{P}}}+J
$$


where $J:=\sum_{0 \leq s \leq .}\left[\Delta X_{s}-h\left(\Delta X_{s}\right)\right], B^{\mathbb{F}_{+}^{\mathbb{P}}}=\int_{0}^{\cdot} b_{s}^{\mathbb{F}_{+}^{\mathbb{P}}} d s$, and $M^{c, \mathbb{F}_{+}^{\mathbb{P}}}, M^{d, \mathbb{F}_{+}^{\mathbb{P}}}$ denote the continuous and purely discontinuous local martingale parts. Moreover, denote by $C^{\mathbb{F}_{+}^{\mathbb{P}}}$ and $F^{\mathbb{F}_{+}^{\mathbb{P}}}(d x) d s$ the second and third $\mathbb{P}-\mathbb{F}_{+}^{\mathbb{P}}$-characteristics of $X$, by $\left[M^{\mathbb{F}_{+}^{\mathbb{P}}}\right]$ the quadratic variation of $\left(M^{c, \mathbb{F}_{+}^{\mathbb{P}}}+M^{d, \mathbb{F}_{+}^{\mathbb{P}}}\right)$, by $\widetilde{C}^{\mathbb{F}_{+}^{\mathbb{P}}}$ the modified second $\mathbb{P}_{-} \mathbb{F}_{+}^{\mathbb{P}}$ characteristic of $X$, and write

$$
\begin{aligned}
U^{i} & :=\int_{0} \int_{\mathbb{R}^{d}} g_{i}(x) \mu^{X}(d x, d s), \\
V^{i, \mathbb{F}_{+}^{\mathbb{P}}}: & =\int_{0} \int_{\mathbb{R}^{d}} g_{i}(x) F^{\mathbb{F}_{+}^{\mathbb{P}}}(d x) d s .
\end{aligned}
$$

We can define the map $\Psi^{\mathbb{P}}: \Omega \rightarrow \bar{\Omega}$ by

$$
\begin{gathered}
\omega \mapsto\left(X(\omega), B^{\mathbb{F}^{\mathbb{P}}}(\omega), M^{c, \mathbb{F}_{+}^{\mathbb{P}}}(\omega), M^{d, \mathbb{F}^{\mathbb{P}}}(\omega), J(\omega), C^{\mathbb{F}^{\mathbb{P}}}(\omega),\left[M^{\mathbb{F}_{+}^{\mathbb{P}}}\right], \widetilde{C}^{\mathbb{F}_{+}^{\mathbb{P}}},\right. \\
\left.\left(U^{i}(\omega), V^{i, \mathbb{F}^{\mathbb{P}}}(\omega)\right)_{i \in \mathbb{N}}\right),
\end{gathered}
$$

which is measurable with respect to the Borel $\sigma$-field, completed by $\mathbb{P}$. Then, the measure

$$
\overline{\mathbb{P}}:=\mathbb{P} \circ\left(\Psi^{\mathbb{P}}\right)^{-1}
$$

is an element of $\overline{\mathfrak{P}}_{\text {sem }}^{a c}$. We used the canonical representation of $X$ with respect to $\mathbb{F}_{+}^{\mathbb{P}}$ to guarantee that for every $\omega$, each summand has càdlàg paths and continuous paths, respectively, and not only $\mathbb{P}$-a.s., so that $\Psi^{\mathbb{P}}$ is well-defined. However, as the characteristics of $X$ do not depend on the choice of $\mathbb{F}$ or $\mathbb{F}_{+}^{\mathbb{P}}$, we conclude that $\mathbb{P} \in \mathfrak{P}_{\text {sem }}^{a c}(\Theta)$ implies $\overline{\mathbb{P}} \in \overline{\mathfrak{P}}_{\text {sem }}^{a c}(\Theta)$; i.e., $\overline{\mathbb{P}}$ preserves the structure of $\mathbb{P}$.

Remark 4.1. By construction, we have

$$
\overline{\mathbb{P}} \circ \bar{X}^{-1}=\mathbb{P} \circ\left(\bar{X} \circ \Psi^{\mathbb{P}}\right)^{-1}=\mathbb{P}=\mathbb{P} \circ X^{-1} .
$$

This implies e.g. that for each $\delta>0$, we have

$$
\begin{aligned}
& \mathbb{E}^{\overline{\mathbb{P}}}\left[\int_{0}^{T} \int_{\{|x| \leq \delta\}}|x|^{2} \bar{F}_{t}^{\overline{\mathbb{P}}}(d x) d t\right]=\mathbb{E}^{\overline{\mathbb{P}}}\left[\sum_{0 \leq t \leq T}\left|\Delta \bar{X}_{t}\right|^{2} \mathbb{1}_{\left\{0<\Delta\left|\bar{X}_{t}\right| \leq \delta\right\}}\right] \\
& =\mathbb{E}^{\mathbb{P}}\left[\sum_{0 \leq t \leq T}\left|\Delta X_{t}\right|^{2} \mathbb{1}_{\left\{0<\Delta\left|X_{t}\right| \leq \delta\right\}}\right] \\
& =\mathbb{E}^{\mathbb{P}}\left[\int_{0}^{T} \int_{\{|x| \leq \delta\}}|x|^{2} F_{t}^{\mathbb{P}}(d x) d t\right],
\end{aligned}
$$

which will play a crucial role in the proof of Theorem 2.1 .

On the other hand, the natural candidate connecting the set $\overline{\mathfrak{P}}_{\text {sem }}^{a c}(\Theta)$ with $\mathfrak{P}_{\text {sem }}^{a c}(\Theta)$ seems to be for any $\overline{\mathbb{P}} \in \overline{\mathfrak{P}}_{\text {sem }}^{a c}(\Theta)$ its corresponding pushforward measure $\mathbb{P}:=\overline{\mathbb{P}} \circ \bar{X}^{-1}$. The following positive answer is stated in such a way that it is compatible with any limit law $\overline{\mathbb{P}}_{0}$ of sequences $\left(\overline{\mathbb{P}}_{n}\right)_{n \in \mathbb{N}} \subseteq \overline{\mathfrak{P}}_{\text {sem }}^{a c}(\Theta)$ with $\Theta$ satisfying Condition (B); see Proposition 3.4. 
Lemma 4.2. Let $\overline{\mathbb{P}} \in \overline{\mathfrak{P}}_{\text {sem }}^{a c, w}$ satisfy

$$
\text { - } \mathbb{E}^{\overline{\mathbb{P}}}\left[\int_{0}^{T}\left[\left|\overline{\bar{b}_{s}^{\mathrm{P}}}\right|+\left|\overline{c_{s}}\right|+\int_{\mathbb{R}^{d}}|h(x)|^{2} \bar{F}_{s}^{\overline{\mathbb{P}}}(d x)\right] d s\right]<\infty,
$$

- both $\bar{M}^{c}, \bar{M}^{d}$ are $\overline{\mathbb{P}}-\overline{\mathbb{F}}$-martingales.

Then, the corresponding pushforward measure

$$
\mathbb{P}:=\overline{\mathbb{P}} \circ(\bar{X})^{-1}
$$

is an element in $\mathfrak{P}_{\text {sem }}^{a c}$. Moreover, if the $\overline{\mathbb{P}}-\overline{\mathbb{F}}$-differential characteristics $\left(\bar{b}^{\overline{\mathbb{P}}}, \bar{c}^{\overline{\mathbb{P}}}, \bar{F}^{\overline{\mathbb{P}}}\right)$ are taking values in some set $\Theta \subseteq \mathbb{R}^{d} \times \mathbb{S}_{+}^{d} \times \mathcal{L}$ which is closed, convex, and satisfies Condition (B), then the corresponding pushforward measure $\mathbb{P}:=\overline{\mathbb{P}} \circ(\bar{X})^{-1}$ is an element in $\mathfrak{P}_{\text {sem }}^{a c}(\Theta)$.

Proof. By Lemma A.1, $\bar{X}$ is a $\overline{\mathbb{P}}-\overline{\mathbb{F}}^{\bar{X}}$-semimartingale with absolutely continuous characteristics, and the local martingale part $\bar{M}^{\bar{X}, \bar{P}}$ of the canonical representation

$$
\bar{X}=\bar{X}_{0}+\bar{B}^{\bar{X}, \overline{\mathbb{P}}}+\bar{M}^{\bar{X}, \overline{\mathbb{P}}}+\sum_{0 \leq s \leq .}\left[\Delta \bar{X}_{s}-h\left(\Delta \bar{X}_{s}\right)\right] \quad \overline{\mathbb{P}} \text {-a.s. }
$$

of $\bar{X}$ under $\overline{\mathbb{P}}-\overline{\mathbb{F}} \bar{X}$ is a $\overline{\mathbb{P}}-\overline{\mathbb{F}}^{\bar{X}}$-martingale. Define the map $\Phi: \Omega \rightarrow \bar{\Omega}$ by $\omega \mapsto$ $(\omega, 0,0,0, \ldots)$. We claim that

$$
\bar{X}_{0} \circ \Phi+\bar{B}^{\bar{X}, \overline{\mathbb{P}}} \circ \Phi+\bar{M}^{\bar{X}, \overline{\mathbb{P}}} \circ \Phi+\sum_{0 \leq s \leq .}\left[\Delta \bar{X}_{s}-h\left(\Delta \bar{X}_{s}\right)\right] \circ \Phi
$$

is the canonical representation of $X$ under $\mathbb{P}-\mathbb{F}$. To see this, observe that each summand in (19) is $\mathbb{F}$-adapted, as $\Phi^{-1}(A) \in \mathcal{F}_{t}$ for all $A \in \overline{\mathcal{F}}_{t}^{\bar{X}}$ for each $t \in$ $[0, T]$. In view of [38, Exercise 1.5.6, p. 44], the $\overline{\mathbb{F}}^{\bar{X}}$-adapted process $\bar{B} \bar{X}, \overline{\mathbb{P}}$ admits a representation

$$
\bar{B}_{t}^{\bar{X}, \overline{\mathbb{P}}}=\Lambda\left(t, \bar{X}_{t_{1}}, \bar{X}_{t_{2}}, \ldots\right), \quad t \in[0, T],
$$

where $\Lambda$ is a measurable function defined on the product space $[0, T] \times\left(\mathbb{R}^{d}\right)^{\mathbb{N}}$ and $0 \leq t_{1}<t_{2}<\cdots$ is a sequence in $[0, T]$. This implies that for all $\bar{\omega} \in \bar{\Omega}$ and $t \in[0, T]$, it holds that

$$
\bar{B}_{t}^{\bar{X}, \overline{\mathbb{P}}}(\bar{\omega})=\bar{B}_{t}^{\bar{X}, \overline{\mathbb{P}}} \circ \Phi \circ \bar{X}(\bar{\omega}) .
$$

In particular, we obtain that

(20) the law of $B^{\bar{X}, \overline{\mathbb{P}}} \circ \Phi$ under $\mathbb{P}=$ the law of $B^{\bar{X}, \overline{\mathbb{P}}}$ under $\overline{\mathbb{P}}$;

hence $B^{\bar{X}, \overline{\mathbb{P}}} \circ \Phi$ is absolutely continuous $\mathbb{P}$-a.s. Of course, (20) also holds with respect to the other summands in (19). In particular, $\bar{M}^{\bar{X}, \bar{P}} \circ \Phi$ is a $\mathbb{P}-\mathbb{F}$-martingale and (19) is $\mathbb{P}$-a.s. equal to $\bar{X} \circ \Phi=X$; hence (19) is indeed the canonical representation of $X$ under $\mathbb{P}-\mathbb{F}$.

Next, from the canonical representation, we see that $B^{\bar{X}, \overline{\mathbb{P}}} \circ \Phi$ is the first characteristic of $X$ under $\mathbb{P}-\mathbb{F}$, which we argued above to be $\mathbb{P}$-a.s. absolutely continuous. Denote by $\nu^{\mathbb{P}}(d x, d t)$ the third characteristic of $X$ under $\mathbb{P}-\mathbb{F}$. Applying (20) to the third characteristic $\nu^{\bar{X}, \overline{\mathbb{P}}}$ of $\bar{X}$ under $\overline{\mathbb{P}}-\overline{\mathbb{F}}^{\bar{X}}$ yields that for any $g_{i} \in \mathcal{C}^{+}\left(\mathbb{R}^{d}\right)$,

(21) the law of $g_{i}(x) * \nu^{\bar{X}, \overline{\mathbb{P}}} \circ \Phi$ under $\mathbb{P}=$ the law of $g_{i}(x) * \nu^{\bar{X}, \overline{\mathbb{P}}}$ under $\overline{\mathbb{P}}$. 
As a consequence, we have for each $g_{i} \in \mathcal{C}^{+}\left(\mathbb{R}^{d}\right)$ that

$$
g_{i}(x) * \nu^{\mathbb{P}}(d x, d t)=g_{i}(x) * \nu^{\bar{X}, \overline{\mathbb{P}}} \circ \Phi,
$$

which implies that $\nu^{\mathbb{P}}$ satisfies a disintegration $\nu^{\mathbb{P}}(d x, d t)=F_{t}^{\mathbb{P}}(d x) d t \mathbb{P}$-a.s. For the second characteristic $C^{\mathbb{P}}$ of $X$ under $\mathbb{P}-\mathbb{F}$, observe that $X=\bar{X} \circ \Phi$ implies the same for the quadratic variation, namely, $[X]=[\bar{X}] \circ \Phi$. As the second characteristic is the continuous part of the quadratic variation as finite variation process (see e.g. [31, Proposition 6.6]), we obtain that $C^{\mathbb{P}}=\bar{C}^{\bar{X}, \overline{\mathbb{P}}} \circ \Phi$, where $\bar{C}^{\bar{X}, \bar{P}}$ denotes the second characteristic of $\bar{X}$ under $\overline{\mathbb{P}}-\overline{\mathbb{F}}$ (which coincides with the one with respect to $\overline{\mathbb{P}}-\overline{\mathbb{F}}$ ). Therefore, applying (20) as above, but with respect to the second characteristic, yields that $C^{\mathbb{P}}$ is absolutely continuous $\mathbb{P}$-a.s. We conclude that $\mathbb{P} \in \mathfrak{P}_{\text {sem }}^{a c}$.

Now, for the rest of the proof, assume that in addition $\overline{\mathbb{P}} \in \overline{\mathfrak{P}}_{\text {sem }}^{a c}(\Theta)$ for some $\Theta \subseteq \mathbb{R}^{d} \times \mathbb{S}_{+}^{d} \times \mathcal{L}$ which is closed, convex, and satisfies Condition (B). From the above arguments, we see that $X$ is a $\mathbb{P}-\mathbb{F}$ semimartingale with differential characteristics

$$
\left(b^{\mathbb{P}}, c^{\mathbb{P}}, F^{\mathbb{P}}\right)=\left(\bar{b}^{\bar{X}, \overline{\mathbb{P}}} \circ \Phi, \bar{c}^{\bar{X}, \overline{\mathbb{P}}} \circ \Phi, \bar{F}^{\bar{X}, \overline{\mathbb{P}}} \circ \Phi\right),
$$

where $\left(\bar{b}^{\bar{X}, \mathbb{P}}, \bar{c}^{X}, \mathbb{P}, \bar{F}^{\bar{X}, \mathbb{P}}\right)$ denotes the $\overline{\mathbb{P}}-\overline{\mathbb{F}}^{\bar{X}}$-differential characteristics of $\bar{X}$. To see that the differential characteristics are taking values in $\Theta \mathbb{P} \times d t$-a.s., we recall the map $\bar{\varphi}: \mathbb{R}^{d} \times \mathbb{S}_{+}^{d} \times \mathcal{L} \rightarrow \mathbb{R}^{d} \times \mathbb{S}_{+}^{d} \times \mathbb{R}^{\mathbb{N}}$ defined in (9) (with corresponding function $\varphi$; see (8) ). By definition of the map $\bar{\varphi}$, (21), and Lemma A.1, we obtain that

$$
\begin{aligned}
& \mathbb{E}^{\mathbb{P}}\left[\int_{0}^{T} \mathbb{1}_{\left\{\bar{\varphi}\left(\left(b_{t}^{\mathbb{P}}, c_{t}^{\mathbb{P}}, F_{t}^{\mathbb{P}}\right)\right) \in \operatorname{cl}(\bar{\varphi}(\Theta))\right\}} d t\right] \\
& =\mathbb{E}^{\mathbb{P}}\left[\int_{0}^{T} \mathbb{1}_{\left\{\left(\bar{b}_{t}^{\bar{X}, \overline{\mathbb{P}}} \circ \Phi, \bar{c}_{t}^{\bar{X}, \overline{\mathbb{P}}} \circ \Phi, \varphi\left(\bar{F}_{t}^{\bar{X}, \overline{\mathbb{P}}} \circ \Phi\right)\right) \in \operatorname{cl}(\bar{\varphi}(\Theta))\right\}} d t\right] \\
& =\mathbb{E}^{\overline{\mathbb{P}}}\left[\int_{0}^{T} \mathbb{1}_{\left\{\left(\bar{b}_{t}^{\bar{X}, \overline{\mathbb{P}}}, \bar{c}_{t}^{\bar{X}, \bar{P}}, \varphi\left(\bar{F}_{t}^{\bar{X}, \overline{\mathbb{P}}}\right)\right) \in \operatorname{cl}(\bar{\varphi}(\Theta))\right\}} d t\right] \\
& =\mathbb{E}^{\overline{\mathbb{P}}}\left[\int_{0}^{T} \mathbb{1}_{\left\{\bar{\varphi}\left(\left(\bar{b}_{t}^{\bar{X}}, \overline{\mathbb{P}}, \bar{c}_{t}^{\bar{X}, \overline{\mathbb{P}}}, \bar{F}_{t}^{\bar{X}, \overline{\mathbb{P}}}\right)\right) \in \operatorname{cl}(\bar{\varphi}(\Theta))\right\}} d t\right] \\
& =T \text {. }
\end{aligned}
$$

Using the property (11) of the map $\bar{\varphi}$, we conclude that $\mathbb{P}:=\overline{\mathbb{P}} \circ(\bar{X})^{-1} \in \mathfrak{P}_{\text {sem }}^{a c}(\Theta)$.

Let us continue with the following lemma.

Lemma 4.3. Let $\left(\mathbb{P}_{n}\right) \subseteq \mathfrak{P}_{\text {sem }}^{a c}$ and consider the corresponding sequence $\left(\overline{\mathbb{P}}_{n}\right) \subseteq$ $\overline{\mathfrak{P}}_{\text {sem }}^{\text {ac }}$ on the enlarged space $\bar{\Omega}$ defined by (17). Assume that both sequences $\left(\mathbb{P}_{n}\right)$ and $\left(\overline{\mathbb{P}}_{n}\right)$ converge to some law $\mathbb{P}_{0} \in \mathfrak{M}_{1}(\Omega)$ and $\overline{\mathbb{P}}_{0} \in \mathfrak{M}_{1}(\bar{\Omega})$, respectively. Then, we have

$$
\mathbb{P}_{0}=\overline{\mathbb{P}}_{0} \circ \bar{X}^{-1} \text {. }
$$

Proof. To see this, observe first that by definition (17), we have for any $n$ that

$$
\overline{\mathbb{P}}_{n} \circ \bar{X}^{-1}=\mathbb{P}_{n} \circ\left(\bar{X} \circ \Psi^{\mathbb{P}}\right)^{-1}=\mathbb{P}_{n} .
$$

Therefore, for any bounded continuous function $g \in C_{b}(\Omega)$, we obtain that

$$
\int_{\Omega} g d \mathbb{P}_{n}=\int_{\bar{\Omega}} g \circ \bar{X} d \overline{\mathbb{P}}_{n}
$$


for every $n$. Consequently, as $\overline{\mathbb{P}}_{n}$ converges to $\overline{\mathbb{P}}_{0}$ weakly and as $\bar{X}: \bar{\Omega} \rightarrow \Omega$ is continuous,

$$
\lim _{n \rightarrow \infty} \int_{\Omega} g d \mathbb{P}_{n}=\lim _{n \rightarrow \infty} \int_{\bar{\Omega}} g \circ \bar{X} d \overline{\mathbb{P}}_{n}=\int_{\bar{\Omega}} g \circ \bar{X} d \overline{\mathbb{P}}_{0}=\int_{\Omega} g d\left(\overline{\mathbb{P}}_{0} \circ \bar{X}^{-1}\right) .
$$

As $g \in C_{b}(\Omega)$ was arbitrary, we conclude that $\overline{\mathbb{P}}_{0} \circ \bar{X}^{-1}$ is the weak limit of $\left(\mathbb{P}_{n}\right)_{n \in \mathbb{N}}$.

Due to Lemma 4.3, we can identify the structure of limit laws of sequences in $\mathfrak{P}_{\text {sem }}^{a c}(\Theta)$.

Proposition 4.4. Let $\Theta \subseteq \mathbb{R}^{d} \times \mathbb{S}_{+}^{d} \times \mathcal{L}$ satisfy Condition (B) and let $\left(\mathbb{P}_{n}\right)_{n \in \mathbb{N}} \subseteq$ $\mathfrak{P}_{\text {sem }}^{a c}(\Theta)$ converge to some law $\mathbb{P}_{0} \in \mathfrak{M}_{1}(\Omega)$. Then the following holds true:

(1) We obtain that $\mathbb{P}_{0} \in \mathfrak{P}_{\text {sem }}^{a c}$.

If, in addition, $\Theta$ is closed, convex, and satisfies Condition $(\mathrm{J})$, then the following holds true:

(2) We obtain that $\mathbb{P}_{0} \in \mathfrak{P}_{\text {sem }}^{a c}(\Theta)$. In particular, $\mathfrak{P}_{\text {sem }}^{a c}(\Theta)$ is closed.

Proof. By Prohorov's theorem, $\left(\mathbb{P}_{n}\right)_{n \in \mathbb{N}}$ is tight. Since $X_{0}: \Omega \rightarrow \mathbb{R}^{d}$ is continuous, $\left(\mathbb{P}_{n} \circ X_{0}^{-1}\right)_{n \in \mathbb{N}} \subseteq \mathfrak{M}_{1}\left(\mathbb{R}^{d}\right)$ is tight, too. Consider the corresponding sequence $\left(\overline{\mathbb{P}}_{n}\right)_{n \in \mathbb{N}} \in \overline{\mathfrak{P}}_{\text {sem }}^{a c}(\Theta)$ on the enlarged space $\bar{\Omega}$ defined in (17). By definition, $\left(\overline{\mathbb{P}}_{n} \circ \bar{X}_{0}^{-1}\right)_{n \in \mathbb{N}} \subseteq \mathfrak{M}_{1}\left(\mathbb{R}^{d}\right)$ is tight. Therefore, by Proposition 3.16, we have tightness of $\left(\overline{\mathbb{P}}_{n}\right)_{n \in \mathbb{N}}$; hence there exists a subsequence $\left(\overline{\mathbb{P}}_{n_{k}}\right)_{k \in \mathbb{N}}$ which converges to some $\overline{\mathbb{P}}_{0} \in \mathfrak{M}_{1}(\bar{\Omega})$. both

To prove (1), we can apply Proposition 3.4 to conclude that $\overline{\mathbb{P}}_{0} \in \overline{\mathfrak{P}}_{\text {sem }}^{a c, w}$ satisfies

$$
\begin{aligned}
& -\mathbb{E}^{\overline{\mathbb{P}}_{0}}\left[\int_{0}^{T}\left[\left|\overline{\mathrm{P}}_{s}\right|+\left|\bar{c}_{s}^{\overline{\mathbb{P}}_{0}}\right|+\int_{\mathbb{R}^{d}}|h(x)|^{2} \bar{F}_{s}^{\overline{\mathbb{P}}_{0}}(d x)\right] d s\right]<\infty, \\
& \text { - } \bar{M}^{c}, \bar{M}^{d} \text { are } \overline{\mathbb{P}_{0}}-\overline{\mathbb{F}} \text {-martingales. }
\end{aligned}
$$

By Lemma 4.2, we obtain that $\overline{\mathbb{P}}_{0} \circ \bar{X}^{-1} \in \mathfrak{P}_{\text {sem }}^{a c}$. Moreover, we know from Lemma 4.3 that $\mathbb{P}_{0}=\overline{\mathbb{P}}_{0} \circ \bar{X}^{-1}$; hence $\mathbb{P}_{0} \in \mathfrak{P}_{\text {sem }}^{a c}$.

To show (2), assume from now on that $\Theta$ is closed, convex, and satisfies Condition $(\mathrm{J})$. Then by Corollary $3.2, \overline{\mathbb{P}}_{0} \in \overline{\mathfrak{P}}_{\text {sem }}^{a c}(\Theta)$. Therefore, we obtain the desired results directly from Lemmas 4.2 and 4.3 .

Next, we present the proofs of Theorem 2.1 and Corollary 2.2. characterizing when a limit law of purely discontinuous martingales remains a purely discontinuous martingale law.

Proof of Theorem 2.1. By Proposition 4.4, $\mathbb{P}_{0} \in \mathfrak{P}_{\text {sem }}^{a c}$; hence it remains to prove that $\mathbb{P}_{0}$ is a martingale law and the characterization is a purely discontinuous martingale law.

Step 1 . We show that the canonical process $X$ on $\Omega$ is a $\mathbb{P}_{0}-\mathbb{F}$-martingale.

To see this, consider the corresponding sequence $\left(\overline{\mathbb{P}}_{n}\right)_{n \in \mathbb{N}} \in \overline{\mathfrak{P}}_{m, d}^{a c}(\Theta)$ on the enlarged space $\bar{\Omega}$ defined by (17). By the same argument as in the beginning of the proof of Proposition 4.4, we obtain the existence of a subsequence $\left(\overline{\mathbb{P}}_{n_{k}}\right)_{k \in \mathbb{N}}$ which converges weakly to some law $\overline{\mathbb{P}}_{0} \in \mathfrak{M}_{1}(\bar{\Omega})$. We deduce from Proposition 3.4 
that the first coordinate $\bar{X}$ is a $\overline{\mathbb{P}}_{0}-\overline{\mathbb{F}}$-semimartingale having absolutely continuous characteristics and canonical representation

$$
\bar{X}=\bar{X}_{0}+\bar{B}+\bar{M}^{c}+\bar{M}^{d}+\bar{J} \quad \overline{\mathbb{P}}_{0} \text {-a.s. }
$$

Moreover, by assumption, $\mathbb{E}^{\overline{\mathbb{P}}_{0}}\left[\left|\bar{X}_{0}\right|\right]<\infty$.

Now, we claim that $\bar{X}$ is a $\overline{\mathbb{P}} 0-\overline{\mathbb{F}}$-martingale. Since we know by Proposition 3.4 that $\bar{X}$ is a $\overline{\mathbb{P}}_{0}-\overline{\mathbb{F}}$-semimartingale with the above canonical representation, where both $\bar{M}^{c}$ and $\bar{M}^{d}$ are $\overline{\mathbb{P}}_{0}-\overline{\mathbb{F}}$-martingales, it remains to show that

$$
\bar{B}+\bar{J} \quad \text { is a } \overline{\mathbb{P}}_{0}-\overline{\mathbb{F}} \text {-martingale. }
$$

From the Skorokhod representation, we have that both $\left(z^{n, \bar{B}}\right)$ and $\left(z^{n, \bar{J}}\right)$ converge pointwise to $z^{0, \bar{B}}$ and $z^{0, \bar{J}}$, respectively. As each $z^{n, \bar{B}}$ and $z^{0, \bar{B}}$ is continuous, we deduce from [21, Proposition VI.2.2 (a), p. 338] that $z^{n, \bar{B}}+z^{n, \bar{J}}$ converges to $z^{0, \bar{B}}+z^{0, \bar{J}}$. Moreover, we know from the proof of Lemma 3.7 that for each $t \in[0, T]$, both sequences $\left(\bar{M}_{t}^{c} \mid \overline{\mathbb{P}}_{n}\right)$ and $\left(\bar{M}_{t}^{d} \mid \overline{\mathbb{P}}_{n}\right)$ are uniformly integrable, and the same holds true by [32, Lemma 5.2] for the sequence $\left(\bar{X}_{t}-\bar{X}_{0} \mid \overline{\mathbb{P}}_{n}\right)$. Therefore, due to the canonical representation of $\bar{X}$ under each $\overline{\mathbb{P}}_{n}$, we can also conclude that the sequence $\left(\bar{B}_{t}+\bar{J}_{t} \mid \overline{\mathbb{P}}_{n}\right)$ is uniformly integrable for each $t \in[0, T]$. As a consequence, we can apply the same proof as in Lemma 3.7(i), but with respect to $\bar{B}+\bar{J}$ instead of $\bar{M}^{d}$ to derive that $\bar{B}+\bar{J}$ is a $\overline{\mathbb{P}}_{0}-\overline{\mathbb{F}}$-martingale.

Consider the smaller filtration $\overline{\mathbb{F}}^{\bar{X}} \subseteq \overline{\mathbb{F}}$ generated by $\bar{X}$. As $\bar{X}$ is a $\overline{\mathbb{P}}_{0}-\overline{\mathbb{F}}$ martingale being $\overline{\mathbb{F}}^{\bar{X}}$-adapted, it is also a $\overline{\mathbb{P}}_{0}-\overline{\mathbb{F}}^{\bar{X}}$ martingale by the tower property of the conditional expectation. Therefore, by construction, we obtain that under the pushforward measure $\overline{\mathbb{P}}_{0} \circ \bar{X}^{-1}$, the canonical process $X$ on the original space $\Omega$ is a $\overline{\mathbb{P}}_{0} \circ \bar{X}^{-1}$-F-martingale. Moreover, applying Lemma 4.3 yields

$$
\mathbb{P}_{0}=\overline{\mathbb{P}}_{0} \circ \bar{X}^{-1}
$$

hence we get the desired result of Step 1 .

Step 2. We want to characterize when $X$ is a $\mathbb{P}_{0}-\mathbb{F}$-purely discontinuous martingale, i.e.,

$$
\mathbb{P}_{0} \in \mathfrak{P}_{m, d}^{a c} \Longleftrightarrow \lim _{\delta \downarrow 0} \limsup _{n \rightarrow \infty} \mathbb{E}^{\mathbb{P}_{n}}\left[\int_{0}^{T} \int_{\{|x| \leq \delta\}}|x|^{2} F_{t}^{\mathbb{P}_{n}}(d x) d t\right]=0 .
$$

To see this, observe that from the first step, $X$ is a $\mathbb{P}_{0}-\mathbb{F}$-martingale and by (22)

$X$ is a $\mathbb{P}_{0}-\mathbb{F}$-purely discont. martingale

$\Longleftrightarrow \bar{X}$ is a $\overline{\mathbb{P}}_{0^{-}} \overline{\mathbb{F}}^{\bar{X}}$-purely discont. martingale.

We obtained in Step 1 that $\bar{X}$ is a $\overline{\mathbb{P}}_{0}-\overline{\mathbb{F}}$ - and $\overline{\mathbb{P}}_{0}-\overline{\mathbb{F}} \bar{X}$-martingale. Moreover, the quadratic variation $[\bar{X}]$ is $\overline{\mathbb{F}}^{\bar{X}}$-adapted, hence so is its continuous part, which implies that the second characteristics of $\bar{X}$ under $\overline{\mathbb{P}}_{0}-\overline{\mathbb{F}}$ and $\overline{\mathbb{P}}_{0}-\overline{\mathbb{F}}^{\bar{X}}$ are the same. From the fact that a martingale is a purely discontinuous one if and only if its second characteristic vanishes, we obtain that

$\bar{X}$ is a $\overline{\mathbb{P}}_{0}-\overline{\mathbb{F}}^{\bar{X}}$-purely discont. martingale

$\Longleftrightarrow \bar{X}$ is a $\overline{\mathbb{P}}_{0}-\overline{\mathbb{F}}$-purely discont. martingale. 
Now, as $\bar{X}$ is a purely discontinuous $\overline{\mathbb{P}}_{n}-\overline{\mathbb{F}}$-martingale, we have that $\bar{M}^{c}=0 \overline{\mathbb{P}}_{n}$-a.s. for all $n \in \mathbb{N}$. Using the Skorokhod representation, this means that $z^{n, \bar{M}^{c}}=0 \lambda$-a.s. for each $n$. As $z^{n, \bar{M}^{c}}$ converges pointwise to $z^{0, \bar{M}^{c}}$, we also have that $z^{0, \bar{M}^{c}}=0$ $\lambda$-a.s., which means that $\bar{M}^{c}=0 \overline{\mathbb{P}}_{0}$-a.s. Therefore, as from Step 1 we know that $\bar{X}=\bar{X}_{0}+\bar{M}^{c}+\bar{M}^{d}+(\bar{B}+\bar{J}) \overline{\mathbb{P}}_{0}$-a.s., with $(\bar{B}+\bar{J})$ being a $\overline{\mathbb{P}}_{0}-\overline{\mathbb{F}}$-purely discontinuous martingale, we conclude that

$\bar{X}$ is a $\overline{\mathbb{P}_{0}}-\overline{\mathbb{F}}$-purely discont. martingale

$\Longleftrightarrow \bar{M}^{d}$ is a $\overline{\mathbb{P}}_{0}-\overline{\mathbb{F}}$-purely discont. martingale.

In Section 3, we proved in Proposition 3.1 that

$\bar{M}^{d}$ is a $\overline{\mathbb{P}}_{0^{-}}-\overline{\mathbb{F}}$-purely discontinuous martingale

$$
\Longleftrightarrow \lim _{\delta \downarrow 0} \limsup _{n \rightarrow \infty} \mathbb{E}^{\overline{\mathbb{P}}_{n}}\left[\int_{0}^{T} \int_{\{|x| \leq \delta\}}|x|^{2} \bar{F}_{t}^{\overline{\mathbb{P}}_{n}}(d x) d t\right]=0 .
$$

Finally, by definition of the corresponding measures $\left(\overline{\mathbb{P}}_{n}\right)_{n \in \mathbb{N}}$ on the enlarged space, we deduce from Remark 4.1 that

$$
\begin{array}{r}
\lim _{\delta \downarrow 0} \limsup _{n \rightarrow \infty} \mathbb{E}^{\overline{\mathbb{P}}_{n}}\left[\int_{0}^{T} \int_{\{|x| \leq \delta\}}|x|^{2} \bar{F}_{t}^{\overline{\mathbb{P}}_{n}}(d x) d t\right]=0 \\
\Longleftrightarrow \lim _{\delta \downarrow 0} \limsup _{n \rightarrow \infty} \mathbb{E}^{\mathbb{P}_{n}}\left[\int_{0}^{T} \int_{\{|x| \leq \delta\}}|x|^{2} F_{t}^{\mathbb{P}_{n}}(d x) d t\right]=0 .
\end{array}
$$

The desired characterization now follows from the equivalence of the statements (23)-(27).

Proof of Corollary 2.2. For each $\mathbb{P}_{n}$, the purely discontinuous martingale part $M^{d, \mathbb{P}_{n}}$ has $\mathbb{P}_{n}-\mathbb{F}$-differential characteristics $\left(0,0, F^{\mathbb{F}, \mathbb{P}_{n}, M}\right)$ with $F^{\mathbb{F}, \mathbb{P}_{n}, M}=F^{\mathbb{P}_{n}} \circ h^{-1}$, where $F^{\mathbb{P}_{n}}$ denotes the third $\mathbb{P}_{n}$ - $\mathbb{F}$-differential characteristic of the canonical process $X$. In particular, $F^{\mathbb{F}, \mathbb{P}_{n}, M}=F^{\mathbb{P}_{n}}$ on $\{|x| \leq \delta\}$ for any small enough $\delta>0$. Denote by $\mathbb{F}^{M} \subseteq \mathbb{F}^{X}$ the filtration generated by $M^{d, \mathbb{P}_{n}}$. We deduce from Lemma A.1 and Remark A.2 that $M^{d, \mathbb{P}_{n}}$ is also a $\mathbb{P}_{n}-\mathbb{F}^{M}$-purely discontinuous martingale with corresponding differential characteristics $\left(0,0, F^{\mathbb{F}^{M}, \mathbb{P}_{n}, M}\right)$ satisfying for any small enough $\delta>0$

$$
\begin{aligned}
& \mathbb{E}^{\mathbb{P}_{n}}\left[\int_{0}^{T} \int_{\{|x| \leq \delta\}}|x|^{2} F_{t}^{\mathbb{F}^{M}, \mathbb{P}_{n}, M}(d x) d t\right] \\
= & \mathbb{E}^{\mathbb{P}_{n}}\left[\int_{0}^{T} \int_{\{|x| \leq \delta\}}|x|^{2} F_{t}^{\mathbb{F}, \mathbb{P}_{n}, M}(d x) d t\right] \\
= & \mathbb{E}^{\mathbb{P}_{n}}\left[\int_{0}^{T} \int_{\{|x| \leq \delta\}}|x|^{2} F_{t}^{\mathbb{P}_{n}}(d x) d t\right] .
\end{aligned}
$$

Therefore, the result follows directly from Theorem 2.1,

We continue with the proof of Theorem 2.5. which provides a necessary and sufficient condition for $\mathfrak{P}_{s e m}^{a c}(\Theta)\left(\Gamma_{0}\right)$ to be compact.

Proof of Theorem 2.5. We already have proved in Proposition 4.4 that Condition $(\mathrm{J})$ implies closedness of the set $\mathfrak{P}_{\text {sem }}^{a c}(\Theta)$. 
For the other direction, i.e., to see that closedness of $\mathfrak{P}_{s e m}^{a c}(\Theta)$ implies that Condition $(\mathrm{J})$ holds whenever $\Theta$ additionally satisfies the condition in Definition 2.4. we assume that Condition $(\mathrm{J})$ fails and want to conclude that then closedness of $\mathfrak{P}_{\text {sem }}^{a c}(\Theta)$ also fails.

By definition, if Condition $(\mathrm{J})$ fails, then there exist $\varepsilon>0$ and a sequence $\left(F^{m}\right)_{m \in \mathbb{N}} \subseteq \operatorname{proj}_{F}(\Theta)$ such that for each $m \in \mathbb{N}$,

$$
\int_{\left\{|x| \leq \frac{1}{m}\right\}}|x|^{2} F^{m}(d x) \geq \varepsilon .
$$

Denote by $\widehat{c}:=\max \left\{|c| \in \operatorname{proj}_{c}(\Theta)\right\}$. Due to $\Theta$ satisfying the condition in Definition 2.4, there exists for each $m$ an element $b^{m} \in \mathbb{R}^{d}$ such that $\left(b^{m}, \widehat{c}, F^{m}\right) \in \Theta$. Denote by $\mathbb{P}^{m}$ the law where the canonical process $X$ is a Lévy process with corresponding Lévy triplet $\left(b^{m}, \widehat{c}, F^{m}\right)$. By definition, each $\mathbb{P}^{m} \in \mathfrak{P}_{\text {sem }}^{a c}(\Theta)$ with $X_{0}=0 \mathbb{P}^{m}$-a.s. We derive from Corollary 3.22 that the sequence $\left(\mathbb{P}^{m}\right)$ is tight; hence there exists a subsequence $\left(\mathbb{P}^{m_{k}}\right)_{k \in \mathbb{N}}$ which by Proposition 4.4 converges weakly to some law $\mathbb{P}_{0} \in \mathfrak{P}_{\text {sem }}^{a c}$. Our goal is to show that $\mathbb{P}_{0} \notin \mathfrak{P}_{\text {sem }}^{a c}(\Theta)$, which would mean that the closedness of $\mathfrak{P}_{\text {sem }}^{a c}(\Theta)$ fails.

To that end, consider the corresponding sequence $\left(\overline{\mathbb{P}}^{k}\right) \subseteq \overline{\mathfrak{P}}_{s e m}^{a c}(\Theta)$ of $\left(\mathbb{P}^{m_{k}}\right)$ on the enlarged space $\bar{\Omega}$ defined by (17). Due to Proposition 3.16 $\left(\overline{\mathbb{P}}^{k}\right)$ is tight; hence there exists a subsequence $\left(\overline{\mathbb{P}}^{k_{u}}\right)_{u \in \mathbb{N}}$ which by Proposition 3.4 converges weakly to some law $\overline{\mathbb{P}}_{0} \in \overline{\mathfrak{P}}_{\text {sem }}^{a c, w}$.

Recall $\bar{c}$ being the differential of $\bar{C}$, which is the quadratic variation of $\bar{M}^{c}$ under $\overline{\mathbb{P}}_{0}$. Morevover, denote by $\bar{c}^{\overline{\mathbb{P}}_{0}, \bar{M}^{d}}$ the differential of the quadratic variation of the continuous martingale part of $\bar{M}^{d}$ under $\overline{\mathbb{P}}_{0^{-}} \overline{\mathbb{F}}$ (note that under $\overline{\mathbb{P}}_{0^{-}} \overline{\mathbb{F}}, \bar{M}^{d}$ is not necessarily a purely discontinuous martingale). Then, the differential $\bar{c}^{\overline{\mathbb{P}}_{0}}$ of the second characteristic of $\bar{X}$ under $\overline{\mathbb{P}}_{0}-\overline{\mathbb{F}}$ satisfies

$$
\bar{c}^{\overline{\mathbb{P}}_{0}}=\bar{c}+\bar{c}^{\overline{\mathbb{P}}_{0}, \bar{M}^{d}} \quad \overline{\mathbb{P}}_{0} \times d t \text {-a.s. }
$$

Observe that by construction of the sequence $\left(\overline{\mathbb{P}}^{k_{u}}\right)$, we have

$$
\begin{aligned}
& \lim _{\delta \downarrow 0} \limsup _{u \rightarrow \infty} \mathbb{E}^{\overline{\mathbb{P}}_{k_{u}}}\left[\int_{0}^{T} \int_{\{|x| \leq \delta\}}|x|^{2} \bar{F}_{t} \overline{\mathbb{P}}_{k_{u}}(d x) d t\right] \\
= & \lim _{\delta \downarrow 0} \limsup _{u \rightarrow \infty} \int_{0}^{T} \int_{\{|x| \leq \delta\}}|x|^{2} \bar{F}^{m_{k_{u}}}(d x) d t \\
\geq & \varepsilon .
\end{aligned}
$$

Therefore, we deduce from Proposition 3.1 that $\bar{M}^{d}$ is not a $\overline{\mathbb{P}_{0}}-\overline{\mathbb{F}}$-purely discontinuous martingale, which implies that $\left(\overline{\mathbb{P}}_{0} \times d t\right)\left[\bar{c}^{\overline{\mathbb{P}}_{0}, \bar{M}^{d}}>0\right]>0$.

Now, we claim that $\bar{c}=\hat{c} \overline{\mathbb{P}}_{0} \times d t$-a.s. To see this, we know from the Skorokhod representation that the sequence $\left(z^{k_{u}, \bar{C}}\right)_{u \in \mathbb{N}}$ converges uniformly to $z^{0, \bar{C}}$. But by definition of the sequence $\left(\overline{\mathbb{P}}^{k_{u}}\right)$, we have $\lambda$-a.s. that $z_{t}^{k_{u}, \bar{C}}=\widehat{c t}, t \in[0, T]$, for all $u$. Therefore, we also have $\lambda$-a.s. that $z_{t}^{0, \bar{C}}=\widehat{c} t, t \in[0, T]$, which implies that indeed $\bar{c}=\hat{c} \overline{\mathbb{P}}_{0} \times d t$-a.s. 
As the second characteristic of $\bar{X}$ under $\overline{\mathbb{P}}-\overline{\mathbb{F}}^{\bar{X}}$ coincides with the one under $\overline{\mathbb{P}}-\overline{\mathbb{F}}$, we obtain that the second differential characteristic $\bar{c}^{X, \overline{\mathbb{P}}_{0}}$ under $\overline{\mathbb{P}}-\overline{\mathbb{F}}^{\bar{X}}$ satisfies

$$
\bar{c} \bar{X}, \overline{\mathbb{P}}_{0}=\bar{c}^{\overline{\mathbb{P}}_{0}}=\widehat{c}+\bar{c}^{\overline{\mathbb{P}}_{0}, \bar{M}^{d}} \overline{\mathbb{P}}_{0} \times d t \text {-a.s. }
$$

Recall that $\widehat{c}:=\max \left\{|c| \in \operatorname{proj}_{c}(\Theta)\right\}$. Due to the positive definiteness, we conclude that

$$
\left(\overline{\mathbb{P}}_{0} \times d t\right)\left[\bar{c}^{\bar{X}, \overline{\mathbb{P}}_{0}} \notin \operatorname{proj}_{c}(\Theta)\right] \geq\left(\overline{\mathbb{P}}_{0} \times d t\right)\left[\bar{c}^{\overline{\mathbb{P}}_{0}, \bar{M}^{d}}>0\right]>0 .
$$

As $\mathbb{P}_{0}=\overline{\mathbb{P}}_{0} \circ \bar{X}^{-1}$, we can argue as in Lemma 4.2 to see that $\mathbb{P}_{0} \in \mathfrak{P}_{\text {sem }}^{a c}$, but $\mathbb{P}_{0} \notin \mathfrak{P}_{\text {sem }}^{a c}(\Theta)$, since the second differential characteristic $c^{\mathbb{P}_{0}}$ satisfies

$$
\left(\mathbb{P}_{0} \times d t\right)\left[c^{\mathbb{P}_{0}} \notin \operatorname{proj}_{c}(\Theta)\right]>0 .
$$

Summarizing, we have shown that if Condition $(\mathrm{J})$ fails, we can find a sequence of probability measures in $\mathfrak{P}_{\text {sem }}^{a c}(\Theta)$ converging to some element $\mathbb{P}_{0}$ which is not an element in $\mathfrak{P}_{\text {sem }}^{a c}(\Theta)$. Hence closedness of $\mathfrak{P}_{\text {sem }}^{a c}(\Theta)$ fails as desired.

Finally, for the compactness criterion, observe that the compactness assumption on $\Gamma_{0} \subseteq \mathfrak{M}_{1}\left(\mathbb{R}^{d}\right)$ implies tightness of $\mathfrak{P}_{\text {sem }}^{a c}(\Theta)\left(\Gamma_{0}\right)$; see Corollary 3.22. Moreover, $\Gamma_{0}$ being closed implies that any sequence $\left(\mathbb{P}_{n}\right) \subseteq \mathfrak{P}_{\text {sem }}^{a c}(\Theta)\left(\Gamma_{0}\right)$ converging to some $\mathbb{P}_{0} \in \mathfrak{M}_{1}(\Omega)$ satisfies $\mathbb{P}_{0} \circ \bar{X}_{0}^{-1} \in \Gamma_{0}$. Hence, the compactness characterization follows directly from the closedness one proved above.

Next, we provide the proof of Theorem 2.8, which is our second compactness criterion for the set $\mathfrak{P}_{s e m}^{a c}(\Theta)\left(\Gamma_{0}\right)$ of semimartingale laws.

Proof of Theorem 2.8. (1) $\Longrightarrow(2)$ : Let $\Gamma_{0} \subseteq \mathfrak{M}_{1}\left(\mathbb{R}^{d}\right)$ be a compact subset of distributions on $\mathbb{R}^{d}$. As by assumption, $\Theta$ satisfies Condition (B), we obtain from Corollary 3.22 that $\mathfrak{P}_{\text {sem }}^{a c}(\Theta)\left(\Gamma_{0}\right)$ is tight. Hence, it remains to show that $\mathfrak{P}_{\text {sem }}^{a c}(\Theta)\left(\Gamma_{0}\right)$ is closed. To that end, let $\left(\mathbb{P}_{n}\right)_{n \in \mathbb{N}} \subseteq \mathfrak{P}_{\text {sem }}^{a c}(\Theta)\left(\Gamma_{0}\right)$ converge to some $\mathbb{P}_{0} \in \mathfrak{M}_{1}(\Omega)$. We need to show that $\mathbb{P}_{0} \in \mathfrak{P}_{\text {sem }}^{a c}(\Theta)\left(\Gamma_{0}\right)$. Consider the corresponding sequence $\left(\overline{\mathbb{P}}_{n}\right)_{n \in \mathbb{N}} \subseteq \overline{\mathfrak{P}}_{\text {sem }}^{a c}(\Theta)\left(\Gamma_{0}\right)$ on the enlarged space $\bar{\Omega}$ defined by (17). By Proposition 3.16, $\overline{\mathfrak{P}}_{\text {sem }}^{a c}(\Theta)\left(\Gamma_{0}\right)$ is tight; hence there exists a subsequence $\left(\overline{\mathbb{P}}_{n_{k}}\right)_{k \in \mathbb{N}}$ which converges weakly to some law $\overline{\mathbb{P}}_{0} \in \mathfrak{M}_{1}(\bar{\Omega})$. Observe that closedness of $\Gamma_{0}$ assures that $\overline{\mathbb{P}}_{0} \circ \bar{X}_{0}^{-1} \in \Gamma_{0}$, whereas Proposition 3.4 implies that $\overline{\mathbb{P}}_{0} \in \overline{\mathfrak{P}}_{\text {sem }}^{a c, w}$. In particular, $\overline{\widetilde{C}}$ is the modified second characteristic of $\bar{X}$ under each $\overline{\mathbb{P}}_{n}-\overline{\mathbb{F}}$ and also under $\overline{\mathbb{P}}_{0}-\overline{\mathbb{F}}$. Following the arguments of the proof of Corollary 3.2 , we see that

$$
\mathbb{E}^{\overline{\mathbb{P}}_{n_{k}}}\left[\int_{0}^{T} \mathbb{1}_{\left\{m\left(\bar{B}_{t+\frac{1}{m}}-\bar{B}_{t}, \overline{\widetilde{C}}_{t+\frac{1}{m}}-\overline{\widetilde{C}}_{t},\left(\bar{V}_{t+\frac{1}{m}}^{i}-\bar{V}_{t}^{i}\right)_{i \in \mathbb{N}}\right) \in \operatorname{cl}(\bar{\varphi}(u(\Theta)))\right\}} d t\right]=T .
$$

In terms of the Skorokhod representation, it means that

$$
\mathbb{E}^{\lambda}\left[\int_{0}^{T} \mathbb{1}_{\left\{m\left(z_{t+\frac{1}{m}}^{n_{k}, \bar{B}}-z_{t}^{n_{k}, \bar{B}}, z_{t+\frac{1}{m}}^{n_{k}, \overline{\widetilde{C}}}-z_{t}^{n_{k}, \overline{\widetilde{C}}},\left(z_{t+\frac{1}{m}}^{n_{k}, \bar{V}^{i}}-z_{t}^{n_{k}, \bar{V}^{i}}\right)_{i \in \mathbb{N}}\right) \in \operatorname{cl}(\bar{\varphi}(u(\Theta)))\right\}} d t\right]=T .
$$

Observe that $\left(z^{n_{k}, \overline{\widetilde{C}}}\right)$ converges to $\left(z^{0, \overline{\widetilde{C}}}\right)$ in $\Omega_{d^{2}}^{c} \lambda$-a.s. Therefore, when letting $k$ tend to infinity, we get that

$$
\mathbb{E}^{\lambda}\left[\int_{0}^{T} \mathbb{1}_{\left\{m\left(z_{t+\frac{1}{m}}^{0, \bar{B}}-z_{t}^{0, \bar{B}}, z_{t+\frac{1}{m}}^{0, \overline{\widetilde{C}}}-z_{t}^{0, \overline{\widetilde{C}}},\left(z_{t+\frac{1}{m}}^{0, \bar{V}^{i}}-z_{t}^{0, \bar{V}^{i}}\right)_{i \in \mathbb{N}}\right) \in \operatorname{cl}(\bar{\varphi}(u(\Theta)))\right\}} d t\right]=T .
$$


This demonstrates that $m\left(\bar{B}_{t+\frac{1}{m}}-\bar{B}_{t}, \overline{\widetilde{C}}_{t+\frac{1}{m}}-\overline{\widetilde{C}}_{t},\left(\bar{V}_{t+\frac{1}{m}}^{i}-\bar{V}_{t}^{i}\right)_{i \in \mathbb{N}}\right) \in \operatorname{cl}(\bar{\varphi}(u(\Theta)))$, $\overline{\mathbb{P}}_{0} \times d t$-a.e. for all $m \in \mathbb{N}$. Now letting $m$ tend to infinity, we obtain that $\left(\bar{b}_{t}, \overline{\widetilde{c}}_{t}, \bar{v}_{t}\right) \in$ $\operatorname{cl}(\bar{\varphi}(u(\Theta)))$ holds $\overline{\mathbb{P}}_{0} \times d t$-a.e., where $\overline{\widetilde{c}}_{t}:=\lim \sup _{n \rightarrow \infty} n\left(\overline{\widetilde{C}}_{t}-\overline{\widetilde{C}}_{\left(t-\frac{1}{n}\right) \vee 0}\right), t \in[0, T]$. Moreover, due to the assumption (1) that $u(\Theta)$ is closed, we can argue as in (11) to obtain the relation

$$
\left(\bar{b}^{\overline{\mathbb{P}}_{0}}, \overline{\vec{c}}^{\overline{\mathbb{P}}_{0}}, \bar{F}^{\overline{\mathbb{P}}_{0}}\right) \in u(\Theta) \quad \overline{\mathbb{P}}_{0} \times d t \text {-a.s. } \quad \Longleftrightarrow(\bar{b}, \overline{\widetilde{c}}, \bar{v}) \in \operatorname{cl}(\bar{\varphi}(u(\Theta))) \quad \overline{\mathbb{P}}_{0} \times d t \text {-a.s. }
$$

This and the definition of the function $u$ ensure that $\left(\bar{b}^{\overline{\mathbb{P}}_{0}}, \overline{\mathrm{P}}^{\overline{\mathbb{P}}_{0}}, \bar{F}^{\overline{\mathbb{P}}_{0}}\right) \in \Theta \overline{\mathbb{P}}_{0} \times d t$-a.s. Therefore, Lemmas 4.2 and 4.3 imply that $\mathbb{P}_{0}=\overline{\mathbb{P}}_{0} \circ \bar{X}^{-1} \in \mathfrak{P}_{\text {sem }}^{a c}(\Theta)\left(\Gamma_{0}\right)$.

$(2) \Longrightarrow(3)$ : Since $\mathfrak{P}_{L}(\Theta) \subseteq \mathfrak{P}_{\text {sem }}^{a c}(\Theta)\left(\left\{\delta_{0}\right\}\right)$, tightness of $\mathfrak{P}_{L}(\Theta)$ follows from the assumption (2) that $\mathfrak{P}_{\text {sem }}^{a c}(\Theta)\left(\left\{\delta_{0}\right\}\right)$ is compact. Therefore, it remains to show that $\mathfrak{P}_{L}(\Theta)$ is closed. To that end, let $\left(\mathbb{P}_{n}\right)_{n \in \mathbb{N}} \subseteq \mathfrak{P}_{\text {sem }}^{a c}(\Theta)\left(\Gamma_{0}\right)$ converge to some $\mathbb{P}_{0} \in \mathfrak{M}_{1}(\Omega)$. Due to assumption $(2), \mathfrak{P}_{\text {sem }}^{a c}(\Theta)\left(\left\{\delta_{0}\right\}\right)$ is compact; hence $\mathbb{P}_{0} \in$ $\mathfrak{P}_{\text {sem }}^{a c}(\Theta)\left(\left\{\delta_{0}\right\}\right)$. Thus, it remains to show that $\mathbb{P}_{0} \in \mathfrak{P}_{L}$. Now, since each $\mathbb{P}_{n} \in \mathfrak{P}_{L}$ is a Lévy law, [21, VII.2.6, p. 395] assures that indeed $\mathbb{P}_{0} \in \mathfrak{P}_{L}$.

$(3) \Longrightarrow(4)$ : Let $\left(\left(b^{n}, c^{n}, F^{n}\right)\right)_{n \in \in \mathbb{N}} \subseteq \Theta$. We need to show that there exists a subsequence $\left(\left(b^{n_{k}}, c^{n_{k}}, F^{n_{k}}\right)\right)_{k \in \mathbb{N}}$ and $\left(b^{0}, c^{0}, F^{0}\right) \in \Theta$ such that for all $f \in C_{b}^{2}\left(\mathbb{R}^{d}\right)$ the subsequence of functions $\left(\left(\mathfrak{L}^{\left(b^{n_{k}}, c^{n_{k}}, F^{n_{k}}\right)} f\right)\right)_{k \in \mathbb{N}}$ converges pointwise to the function $\left(\mathfrak{L}^{\left(b^{0}, c^{0}, F^{0}\right)} f\right)$. To that end, fix any $f \in C_{b}^{2}\left(\mathbb{R}^{d}\right)$. For each $n \in \mathbb{N}$ let $\mathbb{P}_{n} \in \mathfrak{P}_{L}(\Theta)$ be the Lévy law with corresponding Lévy triplet $\left(b^{n}, c^{n}, F^{n}\right)$. Due to assumption $(3), \mathfrak{P}_{L}(\Theta)$ is compact; hence there exists a subsequence $\left(\mathbb{P}_{n_{k}}\right)_{k \in \mathbb{N}}$ which converges to some Lévy law $\mathbb{P}_{0}$ with Lévy triplet $\left(b^{0}, c^{0}, F^{0}\right) \in \Theta$. We claim that the corresponding subsequence of functions $\left(\left(\mathfrak{L}^{\left(b^{n_{k}}, c^{n_{k}}, F^{n_{k}}\right)} f\right)\right)_{k \in \mathbb{N}}$ converges pointwise to the corresponding function $\left(\mathfrak{L}^{\left(b^{0}, c^{0}, F^{0}\right)} f\right)$. To see this, denote by $\widetilde{c}^{n}$ the modified second Lévy triplet for each $n \in \mathbb{N}_{0}$. Then, observe that for each $n \in \mathbb{N}_{0}$, we have for all $x \in \mathbb{R}^{d}$ that

$$
\begin{aligned}
& \left(\mathfrak{L}^{\left(b^{n}, c^{n}, F^{n}\right)} f\right)(x) \\
= & \sum_{i=1}^{d} b^{n, i} \frac{\partial f}{\partial x^{i}}(x)+\frac{1}{2} \sum_{i, j=1}^{d} c^{n, i j} \frac{\partial^{2} f}{\partial x^{i} \partial x^{j}}(x) \\
& +\int_{\mathbb{R}^{d}}\left(f(x+y)-f(x)-\sum_{i=1}^{d} \frac{\partial f}{\partial x^{i}}(x) h^{i}(y)\right) F^{n}(d y) \\
= & \sum_{i=1}^{d} b^{n, i} \frac{\partial f}{\partial x^{i}}(x)+\frac{1}{2} \sum_{i, j=1}^{d}\left(c^{n, i j}+\int_{\mathbb{R}^{d}} h^{i}(y) h^{j}(y) F^{n}(d y)\right) \frac{\partial^{2} f}{\partial x^{i} \partial x^{j}}(x) \\
& +\int_{\mathbb{R}^{d}}\left(f(x+y)-f(x)-\sum_{i=1}^{d} \frac{\partial f}{\partial x^{i}}(x) h^{i}(y)\right. \\
= & \left.\sum_{i=1}^{d} b^{n, i} \frac{\partial f}{\partial x^{i}}(x)+\frac{1}{2} \sum_{i, j=1}^{d} \widetilde{c}^{n, i j} \frac{\partial^{2} f}{\partial x^{i} \partial x^{j}}(x)+\int_{\mathbb{R}^{d}}^{d} \frac{\partial^{2} f}{\partial x^{i} \partial x^{j}}(x) h^{i}(y) h^{j}(y)\right) F^{n}(d y)
\end{aligned}
$$


where $R_{x}(y)=f(x+y)-f(x)-\sum_{i=1}^{d} \frac{\partial f}{\partial x^{i}}(x) h^{i}(y)-\frac{1}{2} \sum_{i, j=1}^{d} \frac{\partial^{2} f}{\partial x^{i} \partial x^{j}}(x) h^{i}(y) h^{j}(y)$. Since $h$ is a bounded continuous function on $\mathbb{R}^{d}$ with $h(y)=y$ in a neighborhood of the origin and $f \in C_{b}^{2}\left(\mathbb{R}^{d}\right)$, the basic property of Taylor expansion guarantees that $R_{x}$ is a bounded continuous function on $\mathbb{R}^{d}$ satisfying $R_{x}(y)=o\left(|y|^{2}\right)$ as $y \rightarrow 0$. Thus, since the subsequence of Lévy laws $\left(\mathbb{P}_{n_{k}}\right)_{k \in \mathbb{N}}$ converges weakly to the Lévy law $\mathbb{P}_{0}$, 21, Theorem II.2.9, p. 396] guarantees that the sequence $\left(\left(b^{n_{k}}, \widetilde{c}^{n_{k}}, F^{n_{k}}\right)\right)_{k \in \mathbb{N}}$ converges to $\left(b^{0}, \widetilde{c}^{0}, F^{0}\right)$ and the sequence $\left(\int_{\mathbb{R}^{d}} R_{x}(y) F^{n_{k}}(d y)\right)_{k \in \mathbb{N}}$ converges to

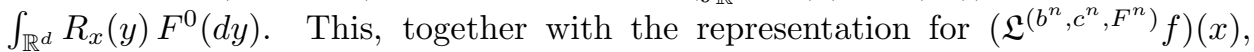
$n \in \mathbb{N}_{0}$, above demonstrates that the subsequence $\left(\mathfrak{L}^{\left(b^{n} k, c^{n_{k}}, F^{n_{k}}\right)} f\right)_{k \in \mathbb{N}}$ indeed converges pointwise to $\left(\mathfrak{L}^{\left(b^{0}, c^{0}, F^{0}\right)} f\right)$.

$(4) \Longrightarrow(5)$ : Fix any $x \in \mathbb{R}^{d}$ and define the function $f_{x}: \mathbb{R}^{d} \rightarrow \mathbb{C}$ by $f_{x}(z):=$ $e^{i x \cdot z}$. Observe that for each Lévy triplet $(b, c, F)$, we have

$$
\psi^{(b, c, F)}(x)=\mathrm{i} x \cdot b-\frac{1}{2} x \cdot c \cdot x+\int_{\mathbb{R}^{d}}\left(\mathrm{e}^{\mathrm{i} x \cdot y}-1-\mathrm{i} x \cdot h(y)\right) F(d y)=\left(L^{(b, c, F)} f_{x}\right)(0) .
$$

Therefore, we see that (5) follows from assumption (4) applied to both the real and imaginary parts of $f_{x}$.

$(5) \Longrightarrow(1):$ Let $\left(\left(b^{n}, \widetilde{c}^{n}, F^{n}\right)\right)_{n \in \mathbb{N}} \subseteq u(\Theta)$ be a sequence which converges to some $(\mathfrak{b}, \widetilde{\mathfrak{c}}, \mathfrak{F}) \in \mathbb{R}^{d} \times \mathbb{S}_{+}^{d} \times \mathcal{L}$. We need to show that there exists $\left(b^{0}, c^{0}, F^{0}\right) \in \Theta$ such that $u\left(b^{0}, c^{0}, F^{0}\right)=(\mathfrak{b}, \widetilde{\mathfrak{c}}, \mathfrak{F})$. To that end, for each $n \in \mathbb{N}$, let $\left(b^{n}, c^{n}, F^{n}\right) \in$ $\Theta$ such that $u\left(b^{n}, c^{n}, F^{n}\right)=\left(b^{n}, \widetilde{c}^{n}, F^{n}\right)$. Moreover, for each $n \in \mathbb{N}$, let $\mu_{n}$ be the infinitely divisible distribution with Lévy triplet $\left(b^{n}, c^{n}, F^{n}\right)$ and characteristic function $\varphi_{\mu_{n}}(x):=e^{\psi^{\left(b^{n}, c^{n}, F^{n}\right)}(x)}$. Consider the sequence of functions $\left(\psi^{\left(b^{n}, c^{n}, F^{n}\right)}\right)_{n \in \mathbb{N}}$. Due to assumption $(5),\left\{\psi^{(b, c, F)}:(b, c, F) \in \Theta\right\}$ is sequentially compact for the topology of pointwise convergence. Therefore, there exists a subsequence of functions $\left(\psi^{\left(b^{n} k, c^{n} k, F^{n}{ }^{n}\right.}\right)_{k \in \mathbb{N}}$ and a Lévy triplet $\left(b^{0}, c^{0}, F^{0}\right) \in \Theta$ such that the subsequence of functions $\left(\psi^{\left(b^{n_{k}}, c^{n_{k}}, F^{n_{k}}\right)}\right)_{k \in \mathbb{N}}$ converges pointwise to the function $\psi^{\left(b^{0}, c^{0}, F^{0}\right)}$. This implies that the subsequence of characteristic functions $\left(e^{\psi^{\left(b^{n} k, c^{n} k, F^{n} k\right)}}\right)_{k \in \mathbb{N}}$ converges pointwise to the characteristic function $e^{\psi^{\left(b^{0}, c^{0}, F^{0}\right)}}$ of the infinitely divisible distribution $\mu_{0}$ with Lévy triplet $\left(b^{0}, c^{0}, F^{0}\right)$. By Lévy's continuity theorem, this implies that the subsequence $\left(\mu_{n_{k}}\right)_{k \in \mathbb{N}}$ converges weakly to $\mu_{0}$. Therefore, [21, Theorem VII.2.9, p. 396] and the definition of the function $u$ in (5) assure that $\left(u\left(b^{n_{k}}, c^{n_{k}}, F^{n_{k}}\right)\right)_{k \in \mathbb{N}}$ also converges to $u\left(b^{0}, c^{0}, F^{0}\right)$. Since by assumption $\left(u\left(b^{n_{k}}, c^{n_{k}}, F^{n_{k}}\right)\right)_{k \in \mathbb{N}}$ also converges to $(\mathfrak{b}, \widetilde{\mathfrak{c}}, \mathfrak{F})$, we obtain that indeed $u\left(b^{0}, c^{0}, F^{0}\right)=(\mathfrak{b}, \widetilde{\mathfrak{c}}, \mathfrak{F})$.

We finish this section with the proof of Example 2.11 showing that in general, Condition $(\mathrm{J})$ is not necessary for $\mathfrak{P}_{\text {sem }}^{a c}(\Theta)$ to be closed.

Proof of Example 2.11. Let $d=1$, and consider the set $\Theta \subseteq \mathbb{R} \times[0, \infty) \times \mathcal{L}$ defined by

$$
\Theta:=\left\{\left.(b, c, F)\left|\operatorname{supp}(F) \subseteq\{|x| \leq 1\}, b=0, c+\int_{\mathbb{R}}\right| x\right|^{2} F(d x)=1\right\} .
$$

Clearly, $\Theta$ is closed, convex, and satisfies Condition $(B)$. We claim that $\mathfrak{P}_{\text {sem }}^{a c}(\Theta)\left(\delta_{0}\right)$ is compact. By (1) in Theorem 2.8, it suffices to show that $u(\Theta) \subseteq \mathbb{R}^{d} \times \mathbb{S}_{+}^{d} \times \mathcal{L}$ is closed. To that end, observe that

$$
u(\Theta)=\{0\} \times\{1\} \times\{F \in \mathcal{L} \mid \operatorname{supp}(F) \subseteq\{|x| \leq 1\}\} .
$$


Therefore, it remains to show that $\{F \in \mathcal{L} \mid \operatorname{supp}(F) \subseteq\{|x| \leq 1\}\} \subseteq \mathcal{L}$ is closed. Let $\left(F_{n}\right)_{n \in \mathbb{N}} \subseteq\{F \in \mathcal{L} \mid \operatorname{supp}(F) \subseteq\{|x| \leq 1\}\}$ converge to some $F_{0} \in \mathcal{L}$. We need to show that $\operatorname{supp}\left(F_{0}\right) \subseteq\{|x| \leq 1\}$. Consider a sequence of functions $\left(f_{k}\right) \subseteq C_{b}(\mathbb{R})$ with values in $[0,1]$ such that $f_{k}=0$ on $\{|x| \leq 1\}$ for each $k$ and $\left(f_{k}\right)$ increasingly converges pointwise to $\mathbb{1}_{\{|x|>1\}}$. Then, by the monotone convergence theorem

$$
\int_{\mathbb{R}^{d}} \mathbb{1}_{\{|x|>1\}} F_{0}(d x)=\lim _{k \rightarrow \infty} \int_{\mathbb{R}^{d}} f_{k}(x) F_{0}(d x) .
$$

Since each $f_{k}$ is a bounded continuous function vanishing in a neighborhood of the origin, we obtain for each $k \in \mathbb{N}$ that

$$
\int_{\mathbb{R}^{d}} f_{k}(x) F_{0}(d x)=\lim _{n \rightarrow \infty} \int_{\mathbb{R}^{d}} f_{k}(x) F_{n}(d x) .
$$

Therefore, as each $F_{n}$ satisfies $\operatorname{supp}\left(F_{n}\right) \subseteq\{|x| \leq 1\}$ and each $f_{k}$ satisfies $f_{k}=0$ on $\{|x| \leq 1\}$, we obtain that

$$
\int_{\mathbb{R}^{d}} \mathbb{1}_{\{|x|>1\}} F_{0}(d x)=\lim _{k \rightarrow \infty} \lim _{n \rightarrow \infty} \int_{\mathbb{R}^{d}} f_{k}(x) F_{n}(d x)=0 .
$$

This implies that $\operatorname{supp}\left(F_{0}\right) \subseteq\{|x| \leq 1\}$.

\section{Semimartingale optimal transport}

The goal of this section is to prove Theorem 2.16. which provides the existence of a minimizer of the primal optimal transport problem introduced in (6), and also present a corresponding duality result. We follow Tan and Touzi 39] and Mikami and Thieullen 30 .

Recall the optimal transport problem (6) defined by

$$
V\left(\mu_{0}, \mu_{1}\right):=\inf _{\mathbb{P} \in \mathfrak{P}_{\Theta}\left(\mu_{0}, \mu_{1}\right)} \mathfrak{J}(\mathbb{P}):=\inf _{\mathbb{P} \in \mathfrak{P}_{\Theta}\left(\mu_{0}, \mu_{1}\right)} \mathbb{E}^{\mathbb{P}}\left[\int_{0}^{1} L\left(t, X, b_{t}^{\mathbb{P}}, c_{t}^{\mathbb{P}}, F_{t}^{\mathbb{P}}\right) d t\right],
$$

where we write $\mathfrak{P}_{\Theta} \equiv \mathfrak{P}_{\text {sem }}^{a c}(\Theta)$ to shorten the notation.

Remark 5.1. Under the assumption on $\Theta$ stated in Theorem 2.16, we have as a consequence of Theorem 2.5 that $\mathfrak{P}_{\Theta}\left(\mu_{0}, \mu_{1}\right)$ is compact. Indeed, tightness is clear as $\mathfrak{P}_{\Theta}\left(\mu_{0}, \mu_{1}\right) \subseteq \mathfrak{P}_{\Theta}$. Closedness follows from the observation that for any sequence $\left(\mathbb{P}_{n}\right) \subseteq \mathfrak{P}_{\Theta}$ converging to some $\mathbb{P}_{0} \in \mathfrak{P}_{\Theta}$, we have the convergence of $\left(\mathbb{P}_{n} \circ X_{0}^{-1}\right)_{n \in \mathbb{N}}$ to $\mathbb{P}_{0} \circ X_{0}^{-1}$, and as $\mathbb{P}_{0}$ has no fixed time of discontinuity, we also have the convergence of $\left(\mathbb{P}_{n} \circ X_{1}^{-1}\right)_{n \in \mathbb{N}}$ to $\mathbb{P}_{0} \circ X_{1}^{-1}$.

We start with a useful lemma which allows us to consider the optimal transport problem introduced in (6), but on the enlarged space introduced in Section 3 , and give its relation to the original one. To that end, recall the function $\bar{\varphi}$ : $\mathbb{R}^{d} \times \mathbb{S}_{+}^{d} \times \mathcal{L} \rightarrow \mathbb{R}^{d} \times \mathbb{S}_{+}^{d} \times \mathbb{R}^{\mathbb{N}}$ introduced in (9) (with corresponding function $\varphi$ ). We have seen that $\bar{\varphi}$ is an additive, positive homogeneous map being a bijection onto its image; see Section 3.1.

Define the corresponding function $\bar{L}:[0,1] \times \Omega \times \bar{\varphi}(\Theta) \rightarrow[0, \infty)$ by setting

$$
\bar{L}(t, \omega, \bar{\varphi}(b, c, F)):=L(t, \omega, b, c, F),
$$


and define for any $\overline{\mathbb{P}} \in \mathfrak{P}_{\Theta}\left(\mu_{0}, \mu_{1}\right)$ the associated transportation cost

$$
\overline{\mathfrak{J}}(\overline{\mathbb{P}}):=\mathbb{E}^{\overline{\mathbb{P}}}\left[\int_{0}^{1} \bar{L}\left(t, \bar{X}, \bar{\varphi}\left(b_{t}^{\overline{\mathbb{P}}}, c_{t}^{\overline{\mathbb{P}}}, F_{t}^{\overline{\mathbb{P}}}\right)\right) d t\right]=\mathbb{E}^{\overline{\mathbb{P}}}\left[\int_{0}^{1} \bar{L}\left(t, \bar{X}, \bar{b}_{t}, \bar{c}_{t}, \bar{v}_{t}\right) d t\right],
$$

where $\bar{b}, \bar{c}, \bar{v}$ are defined as in Section 3.1 .

Lemma 5.2. Under the conditions of Theorem 2.16, the following hold true:

(i) For any $\mathbb{P} \in \mathfrak{P}_{\Theta}\left(\mu_{0}, \mu_{1}\right)$, let $\overline{\mathbb{P}} \in \overline{\mathfrak{P}}_{\Theta}\left(\mu_{0}, \mu_{1}\right)$ be the corresponding measure on the enlarged space $\bar{\Omega}$ defined in (17). Then, we have $\mathfrak{J}(\mathbb{P})=\overline{\mathfrak{J}}(\overline{\mathbb{P}})$.

(ii) Conversely, for any $\overline{\mathbb{P}} \in \overline{\mathfrak{P}}_{\Theta}\left(\mu_{0}, \mu_{1}\right)$, let $\mathbb{P}:=\overline{\mathbb{P}} \circ \bar{X}^{-1} \in \mathfrak{P}_{\Theta}\left(\mu_{0}, \mu_{1}\right)$ be the pushforward measure defined in (18). Then, we have $\overline{\mathfrak{J}}(\overline{\mathbb{P}}) \geq \mathfrak{J}(\mathbb{P})$.

Proof. To see part (i), let $\mathbb{P} \in \mathfrak{P}_{\Theta}\left(\mu_{0}, \mu_{1}\right)$ and let $\overline{\mathbb{P}}:=\mathbb{P} \circ\left(\Psi^{\mathbb{P}}\right)^{-1} \in \overline{\mathfrak{P}}_{\Theta}\left(\mu_{0}, \mu_{1}\right)$, where $\Psi^{\mathbb{P}}: \Omega \rightarrow \bar{\Omega}$ is defined just above (17). Moreover, recall the set $\mathcal{C}^{+}\left(\mathbb{R}^{d}\right):=$ $\left\{g_{i} \mid i \in \mathbb{N}\right\}$ and the processes $\bar{b}, \bar{c}$, and $\bar{v}:=\left(\bar{v}^{1}, \bar{v}^{2}, \ldots\right)$ defined in Section 3.1 . Then,

$$
\begin{aligned}
\mathfrak{J}(\mathbb{P}) & =\mathbb{E}^{\mathbb{P}}\left[\int_{0}^{1} L\left(t, X, b_{t}^{\mathbb{P}}, c_{t}^{\mathbb{P}}, F_{t}^{\mathbb{P}}\right) d t\right] \\
& =\mathbb{E}^{\mathbb{P}}\left[\int_{0}^{1} \bar{L}\left(t, \bar{X}, \bar{b}_{t}^{\overline{\mathbb{P}}}, \bar{c}_{t}^{\overline{\mathbb{P}}},\left(\int_{\mathbb{R}^{d}} g_{i}(x) \bar{F}_{t}^{\overline{\mathbb{P}}}(d x)\right)_{i \in \mathbb{N}}\right) \circ \Psi^{\mathbb{P}} d t\right] \\
& =\mathbb{E}^{\mathbb{P}}\left[\int_{0}^{1} \bar{L}\left(t, \bar{X}, \bar{b}_{t}, \bar{c}_{t}, \bar{v}_{t}\right) \circ \Psi^{\mathbb{P}} d t\right] \\
& =\mathbb{E}^{\overline{\mathbb{P}}}\left[\int_{0}^{1} \bar{L}\left(t, \bar{X}, \bar{b}_{t}, \bar{c}_{t}, \bar{v}_{t}\right) d t\right] \\
& =\overline{\mathfrak{J}}(\overline{\mathbb{P}}) .
\end{aligned}
$$

For part (ii), let $\overline{\mathbb{P}} \in \overline{\mathfrak{P}}_{\Theta}\left(\mu_{0}, \mu_{1}\right)$ and let $\mathbb{P}:=\overline{\mathbb{P}} \circ \bar{X}^{-1} \in \mathfrak{P}_{\Theta}\left(\mu_{0}, \mu_{1}\right)$ be the pushforward measure defined in Lemma 4.2. Denote by $\left(\bar{b}^{\mathbb{P}}, \bar{c}^{\overline{\mathbb{P}}}, \bar{F}^{\overline{\mathbb{P}}}\right)$ the $\overline{\mathbb{P}}$ $\overline{\mathbb{F}}$-differential characteristics of $\bar{X}$. Due to Assumption 2.14 and its definition, the function $\bar{L}(t, \omega, \cdot, \cdot, \cdot)$ is convex on $\bar{\varphi}(\Theta)$ for each $t, \omega$. Denote by $\overline{\mathbb{F}}_{+}^{\bar{X}, \overline{\mathbb{P}}}$ the usual $\overline{\mathbb{P}}_{-}$ augmentation of $\overline{\mathbb{F}}^{\bar{X}}$. Using Fubini's theorem, Jensen inequality, and the definition of $\overline{\mathbb{P}}$ yields

$$
\begin{aligned}
\overline{\mathfrak{J}}(\overline{\mathbb{P}}) & =\int_{0}^{1} \mathbb{E}^{\overline{\mathbb{P}}}\left[\bar{L}\left(t, \bar{X}, \bar{b}_{t}, \bar{c}_{t}, \bar{v}_{t}\right)\right] d t \\
& \geq \int_{0}^{1} \mathbb{E}^{\overline{\mathbb{P}}}\left[\bar{L}\left(t, \bar{X}, \mathbb{E}^{\overline{\mathbb{P}}}\left[\left(\bar{b}_{t}, \bar{c}_{t}, \bar{v}_{t}\right) \mid \overline{\mathcal{F}}_{t+}^{\bar{X}}, \overline{\mathbb{P}}\right]\right)\right] d t \\
& =\int_{0}^{1} \mathbb{E}^{\overline{\mathbb{P}}}\left[\bar{L}\left(t, \bar{X}, \mathbb{E}^{\overline{\mathbb{P}}}\left[\left(\bar{b}_{t}^{\overline{\mathbb{P}}}, \bar{c}_{t}^{\bar{P}},\left(\int_{\mathbb{R}^{d}} g_{i}(x) \bar{F}_{t}^{\overline{\mathbb{P}}}(d x)\right)_{i \in \mathbb{N}}\right) \mid \overline{\mathcal{F}}_{t+}^{\bar{X}, \overline{\mathbb{P}}}\right]\right)\right] d t .
\end{aligned}
$$

By Lemma A.1, the differential characteristics $\left(\bar{b}^{\bar{X}, \overline{\mathbb{P}}}, \bar{c}^{\bar{X}, \overline{\mathbb{P}}}, \bar{F}^{\bar{X}, \overline{\mathbb{P}}}\right)$ of $\bar{X}$ under $\overline{\mathbb{P}}$ $\overline{\mathbb{F}}^{\bar{X}}$ (which are the same under $\overline{\mathbb{P}}-\overline{\mathbb{F}}_{+}^{\bar{X}}, \overline{\mathbb{P}}$ ) are optional projections of the differential characteristics of $\bar{X}$ under $\overline{\mathbb{P}}-\overline{\mathbb{F}}$. Therefore, we obtain from the definition of $\bar{\varphi}$ defined 
in (9) that

$$
\begin{aligned}
& \int_{0}^{1} \mathbb{E}^{\overline{\mathbb{P}}}\left[\bar{L}\left(t, \bar{X}, \mathbb{E}^{\overline{\mathbb{P}}}\left[\left(\bar{b}_{t}^{\overline{\mathbb{P}}}, \bar{c}_{t}^{\overline{\mathbb{P}}},\left(\int_{\mathbb{R}^{d}} g_{i}(x) \bar{F}_{t}^{\overline{\mathbb{P}}}(d x)\right)_{i \in \mathbb{N}}\right) \mid \overline{\mathcal{F}}_{t+}^{\bar{X}, \overline{\mathbb{P}}}\right]\right)\right] d t \\
= & \int_{0}^{1} \mathbb{E}^{\overline{\mathbb{P}}}\left[\bar{L}\left(t, \bar{X}, \bar{b}_{t}^{\bar{X}, \overline{\mathbb{P}}}, \bar{c}_{t}^{\bar{X}, \overline{\mathbb{P}}},\left(\int_{\mathbb{R}^{d}} g_{i}(x) \bar{F}_{t}^{\bar{X}, \overline{\mathbb{P}}}(d x)\right)_{i \in \mathbb{N}}\right)\right] d t \\
= & \int_{0}^{1} \mathbb{E}^{\overline{\mathbb{P}}}\left[\bar{L}\left(t, \bar{X}, \bar{\varphi}\left(\bar{b}_{t}^{\bar{X}, \overline{\mathbb{P}}}, \bar{c}_{t}^{\bar{X}, \overline{\mathbb{P}}}, \bar{F}_{t}^{\bar{X}, \overline{\mathbb{P}}}\right)\right)\right] d t .
\end{aligned}
$$

We know from the proof of Lemma 4.2 that

$$
\begin{aligned}
& \text { the law of } \bar{\varphi}\left(\bar{b}^{\bar{X}, \overline{\mathbb{P}}}, \bar{c}^{\bar{X}}, \overline{\mathbb{P}}, \bar{F}^{\bar{X}, \overline{\mathbb{P}}}\right) \text { under } \overline{\mathbb{P}} \\
= & \text { the law of } \bar{\varphi}\left(\bar{b}^{X, \overline{\mathbb{P}}}, \bar{c}^{X}, \overline{\mathbb{P}}, \bar{F}^{X, \overline{\mathbb{P}}}\right) \circ \Phi \text { under } \mathbb{P}
\end{aligned}
$$

and that the differential characteristics of $X$ under $\mathbb{P}-\mathbb{F}$ satisfy

$$
\left(b^{\mathbb{P}}, c^{\mathbb{P}}, F^{\mathbb{P}}\right)=\left(\bar{b}^{\bar{X}, \overline{\mathbb{P}}} \circ \Phi, \bar{c}^{\bar{X}, \overline{\mathbb{P}}} \circ \Phi, \bar{F}^{\bar{X}, \overline{\mathbb{P}}} \circ \Phi\right) .
$$

Therefore, we conclude that

$$
\begin{aligned}
\int_{0}^{1} \mathbb{E}^{\overline{\mathbb{P}}}\left[\bar{L}\left(t, \bar{X}, \bar{\varphi}\left(\bar{b}_{t}^{\bar{X}, \overline{\mathbb{P}}}, \bar{c}_{t}^{\bar{X}, \overline{\mathbb{P}}}, \bar{F}_{t}^{\bar{X}, \overline{\mathbb{P}}}\right)\right)\right] d t & =\int_{0}^{1} \mathbb{E}^{\mathbb{P}}\left[L\left(t, X, b_{t}^{\mathbb{P}}, c_{t}^{\mathbb{P}}, F_{t}^{\mathbb{P}}\right)\right] d t \\
& =\mathfrak{J}(\mathbb{P}) .
\end{aligned}
$$

Now we are able to prove the existence of a minimizer $\widehat{\mathbb{P}} \in \mathfrak{P}_{\Theta}\left(\mu_{0}, \mu_{1}\right)$ for (66).

Lemma 5.3. The function

$$
\mathfrak{M}_{1}\left(\mathbb{R}^{d}\right) \times \mathfrak{M}_{1}\left(\mathbb{R}^{d}\right) \rightarrow[0, \infty], \quad\left(\mu_{0}, \mu_{1}\right) \mapsto V\left(\mu_{0}, \mu_{1}\right)
$$

is lower semicontinuous. As a consequence, there exists $\widehat{\mathbb{P}} \in \mathfrak{P}_{\Theta}\left(\mu_{0}, \mu_{1}\right)$ satisfying

$$
\mathfrak{J}(\widehat{\mathbb{P}})=\inf _{\mathbb{P} \in \mathfrak{P}_{\Theta}\left(\mu_{0}, \mu_{1}\right)} \mathfrak{J}(\mathbb{P}) .
$$

Proof. We follow [39, Lemma 3.13], which goes back to the arguments in [30, Lemma 3.1].

Let $\left(\mu_{0}^{n}\right)_{n \in \mathbb{N}}$ and $\left(\mu_{1}^{n}\right)_{n \in \mathbb{N}}$ be two sequences in $\mathfrak{M}_{1}\left(\mathbb{R}^{d}\right)$ converging weakly to $\mu_{0}$ and $\mu_{1}$, respectively. We need to show that

$$
\liminf _{n \rightarrow \infty} V\left(\mu_{0}^{n}, \mu_{1}^{n}\right) \geq V\left(\mu_{0}, \mu_{1}\right) .
$$

Without loss of generality, assume that $\liminf _{n \rightarrow \infty} V\left(\mu_{0}^{n}, \mu_{1}^{n}\right)<\infty$. Then, after choosing a subsequence if necessary, we can assume that the sequence $\left(V\left(\mu_{0}^{n}, \mu_{1}^{n}\right)\right)_{n \in \mathbb{N}}$ is bounded, and for each $n \in \mathbb{N}$, there exists a probability measure $\mathbb{P}_{n} \in \mathfrak{P}_{\Theta}\left(\mu_{0}^{n}, \mu_{1}^{n}\right)$ such that

$$
0 \leq \mathfrak{J}\left(\mathbb{P}_{n}\right)-V\left(\mu_{0}^{n}, \mu_{1}^{n}\right) \leq \frac{1}{n}
$$

Thanks to Lemma [5.2, we find for each $n \in \mathbb{N}$ a corresponding measure $\overline{\mathbb{P}}_{n} \in$ $\overline{\mathfrak{P}}_{\Theta}\left(\mu_{0}^{n}, \mu_{1}^{n}\right)$ on the enlarged space satisfying $\overline{\mathfrak{J}}\left(\overline{\mathbb{P}}_{n}\right)=\mathfrak{J}\left(\mathbb{P}_{n}\right)$.

Note that the sequences $\left(\mu_{0}^{n}\right)_{n \in \mathbb{N}}$ and $\left(\mu_{1}^{n}\right)_{n \in \mathbb{N}}$ are tight as they converge weakly to $\mu_{0}$ and $\mu_{1}$, respectively. Consequently, Proposition 3.16 implies tightness of the sequence $\left(\overline{\mathbb{P}}_{n}\right)_{n \in \mathbb{N}}$. Moreover, we deduce from Corollary 3.2 and the arguments in 
Remark 5.1 that any limit law $\overline{\mathbb{P}}_{0}$ of a converging subsequence $\left(\overline{\mathbb{P}}_{n_{k}}\right)_{k \in \mathbb{N}} \subseteq\left(\overline{\mathbb{P}}_{n}\right)_{n \in \mathbb{N}}$ is an element of $\overline{\mathfrak{P}}_{\Theta}\left(\mu_{0}, \mu_{1}\right)$.

Now, as the cost function satisfies Assumption 2.14, we can follow exactly the arguments of [39, Lemma 3.9], which go back to [28], to derive the lower semicontinuity of the map

$$
\overline{\mathfrak{P}}_{\Theta} \rightarrow[0, \infty], \quad \overline{\mathbb{P}} \mapsto \overline{\mathfrak{J}}(\overline{\mathbb{P}}) .
$$

Moreover, by Lemma 5.2 , we can find a probability measure $\mathbb{P}_{0} \in \mathfrak{P}_{\Theta}\left(\mu_{0}, \mu_{1}\right)$ such that $\mathfrak{J}\left(\mathbb{P}_{0}\right) \leq \overline{\mathfrak{J}}\left(\overline{\mathbb{P}}_{0}\right)$. Hence, we obtain the lower semicontinuity due to the inequalities

$$
\begin{aligned}
\liminf _{n \rightarrow \infty} V\left(\mu_{0}^{n}, \mu_{1}^{n}\right)=\liminf _{n \rightarrow \infty} \mathfrak{J}\left(\mathbb{P}_{n}\right)=\liminf _{n \rightarrow \infty} \overline{\mathfrak{J}}\left(\overline{\mathbb{P}}_{n}\right) \geq \overline{\mathfrak{J}}\left(\overline{\mathbb{P}}_{0}\right) & \geq \mathfrak{J}\left(\mathbb{P}_{0}\right) \\
& \geq V\left(\mu_{0}, \mu_{1}\right) .
\end{aligned}
$$

To obtain the existence of a minimizer $\widehat{\mathbb{P}} \in \mathfrak{P}_{\Theta}\left(\mu_{0}, \mu_{1}\right)$, choose $\left(\mu_{0}^{n}, \mu_{1}^{n}\right)=$ $\left(\mu_{0}, \mu_{1}\right)$ and follow the arguments used above from equation (29) on to derive the result.

In the rest of this section, we prove the duality result for the value function $V\left(\mu_{0}, \mu_{1}\right)$ stated in Theorem 2.16. We will use classical convex duality arguments, which require that $V\left(\mu_{0}, \mu_{1}\right)$ be lower semicontinuous and convex. Whereas the lower semicontinuity of $V\left(\mu_{0}, \mu_{1}\right)$ was already shown in Lemma 5.3. we can argue exactly the same way as in [39, Lemma 3.15] to obtain the convexity of the map

$$
\mathfrak{M}_{1}\left(\mathbb{R}^{d}\right) \times \mathfrak{M}_{1}\left(\mathbb{R}^{d}\right) \rightarrow[0, \infty], \quad\left(\mu_{0}, \mu_{1}\right) \mapsto V\left(\mu_{0}, \mu_{1}\right) .
$$

Before starting the proof of the duality result, recall the dual function

$$
\mathcal{V}\left(\mu_{0}, \mu_{1}\right):=\sup _{\lambda_{1} \in C_{b}\left(\mathbb{R}^{d}\right)}\left\{\int_{\mathbb{R}^{d}} \lambda_{0}^{\lambda_{1}}(x) \mu_{0}(d x)-\int_{\mathbb{R}^{d}} \lambda_{1}(x) \mu_{1}(d x)\right\},
$$

where

$$
\lambda_{0}^{\lambda_{1}}(x):=\inf _{\mathbb{P} \in \mathfrak{P}_{\Theta}\left(\delta_{x}\right)} \mathbb{E}^{\mathbb{P}}\left[\int_{0}^{1} L\left(t, X, b_{t}^{\mathbb{P}}, c_{t}^{\mathbb{P}}, F_{t}^{\mathbb{P}}\right) d t+\lambda_{1}\left(X_{1}\right)\right] .
$$

By arguing exactly as in the proof of [39, Lemma 3.5] (using [32, Theorem 2.1] for the conditioning and pasting of probability measures), we get immediately the following result.

Lemma 5.4. Let the cost function $L$ satisfy Assumption 2.14, Then for any $\lambda_{1} \in$ $C_{b}\left(\mathbb{R}^{d}\right)$, the function $\lambda_{0}^{\lambda_{1}}$ is measurable with respect to the Borel $\sigma$-field on $\mathbb{R}^{d}$ completed by $\mu_{0}$, and

$$
\int_{\mathbb{R}^{d}} \lambda_{0}^{\lambda_{1}}(x) \mu_{0}(d x)=\inf _{\mathbb{P} \in \mathfrak{P}_{\Theta}\left(\mu_{0}\right)} \mathbb{E}^{\mathbb{P}}\left[\int_{0}^{1} L\left(t, X, b_{t}^{\mathbb{P}}, c_{t}^{\mathbb{P}}, F_{t}^{\mathbb{P}}\right) d t+\lambda_{1}\left(X_{1}\right)\right] .
$$

In particular, the integral $\int_{\mathbb{R}^{d}} \lambda_{0}^{\lambda_{1}}(x) \mu_{0}(d x)$ is well-defined.

To keep the notation short, denote $\mu(\phi):=\int_{\mathbb{R}^{d}} \phi(x) \mu(d x)$ for all $\mu \in \mathfrak{M}_{1}\left(\mathbb{R}^{d}\right)$, $\phi \in L^{1}(\mu)$.

Proof of Theorem 2.16. In Lemma 5.3, we already have proved the existence of a minimizer $\widehat{\mathbb{P}} \in \mathfrak{P}_{\Theta}\left(\mu_{0}, \mu_{1}\right)$ of the primal optimal transport problem (6) whenever $V\left(\mu_{0}, \mu_{1}\right)<\infty$.

To obtain the duality result, we follow the argument of [39, p. 9] and [30, Theorem 2.1]. For any fixed initial distribution $\mu_{0}$, observe that if $V\left(\mu_{0}, \mu_{1}\right)=\infty$ for 
every $\mu_{1} \in \mathfrak{M}_{1}\left(\mathbb{R}^{d}\right)$, then $\mathfrak{J}(\mathbb{P})=\infty$ for all $\mathbb{P} \in \mathfrak{P}_{\Theta}\left(\mu_{0}\right)$, which by definition of the dual function and Lemma 5.4 implies that also $\mathcal{V}\left(\mu_{0}, \mu_{1}\right)=\infty$. In this case, the duality result holds true trivially.

Now, consider the case where the function $\mu_{1} \mapsto V\left(\mu_{0}, \mu_{1}\right)$ is not always equal to infinity. Denote by $\mathfrak{M}_{f, s}\left(\mathbb{R}^{d}\right)$ the space of all finite signed measures on $\mathbb{R}^{d}$ equipped with the coarsest topology making the maps $\mu \mapsto \mu(\phi)$ continuous for every $\phi \in C_{b}\left(\mathbb{R}^{d}\right)$. Then, the subspace topology on $\mathfrak{M}_{1}\left(\mathbb{R}^{d}\right)$ coincides with the usual weak topology on it; see [7, Chapter 8]. By extending $V\left(\mu_{0}, \cdot\right)$ from $\mathfrak{M}_{1}\left(\mathbb{R}^{d}\right)$ to $\mathfrak{M}_{f, s}\left(\mathbb{R}^{d}\right)$ setting $V\left(\mu_{0}, \mu_{1}\right)=\infty$ for all $\mu_{1} \in \mathfrak{M}_{f, s}\left(\mathbb{R}^{d}\right) \backslash \mathfrak{M}_{1}\left(\mathbb{R}^{d}\right)$, we retain its lower semicontinuity and convexity also on the bigger space $\mathfrak{M}_{f, s}\left(\mathbb{R}^{d}\right)$.

Now, recall that the dual space $\mathfrak{M}_{f, s}\left(\mathbb{R}^{d}\right)^{*}$ of $\mathfrak{M}_{f, s}\left(\mathbb{R}^{d}\right)$ is defined by

$$
\mathfrak{M}_{f, s}\left(\mathbb{R}^{d}\right)^{*}=\left\{\mu \mapsto \int_{\mathbb{R}^{d}} \phi(x) \mu(d x) \mid \phi \in C_{b}\left(\mathbb{R}^{d}\right)\right\}
$$

(see e.g. [10, Lemma 3.2.3, p. 65]) and the Legendre transform $g: C_{b}\left(\mathbb{R}^{d}\right) \rightarrow$ $(-\infty, \infty]$ of $V\left(\mu_{0}, \cdot\right)$ defined by

$$
g\left(\lambda_{1}\right):=\sup _{\mu_{1} \in \mathfrak{M}_{f, s}\left(\mathbb{R}^{d}\right)}\left\{\mu_{1}\left(\lambda_{1}\right)-V\left(\mu_{0}, \mu_{1}\right)\right\} .
$$

We can apply the classical convex duality result [10, Theorem 2.2.15, p. 55] to get

$$
V\left(\mu_{0}, \mu_{1}\right)=\sup _{\lambda_{1} \in C_{b}\left(\mathbb{R}^{d}\right)}\left\{\mu_{1}\left(\lambda_{1}\right)-g\left(\lambda_{1}\right)\right\} .
$$

Moreover, using the definition of $V\left(\mu_{0}, \mu_{1}\right)$ and Lemma 5.4 leads to the following characterization of the Legendre transform:

$$
\begin{aligned}
g\left(-\lambda_{1}\right) & :=\sup _{\mu_{1} \in \mathfrak{M}_{f, s}\left(\mathbb{R}^{d}\right)}\left\{\mu_{1}\left(-\lambda_{1}\right)-V\left(\mu_{0}, \mu_{1}\right)\right\} \\
& =\sup _{\mu_{1} \in \mathfrak{M}_{1}\left(\mathbb{R}^{d}\right)}\left\{\mu_{1}\left(-\lambda_{1}\right)-V\left(\mu_{0}, \mu_{1}\right)\right\} \\
& =-\inf _{\mu_{1} \in \mathfrak{M}_{1}\left(\mathbb{R}^{d}\right)} \inf _{\mathbb{P} \in \mathfrak{P}_{\Theta}\left(\mu_{0}, \mu_{1}\right)}\left\{\mathbb{E}^{\mathbb{P}}\left[\lambda_{1}\left(X_{1}\right)\right]+\mathfrak{J}(\mathbb{P})\right\} \\
& =-\inf _{\mathbb{P} \in \mathfrak{P}_{\Theta}\left(\mu_{0}\right)}\left\{\mathbb{E}^{\mathbb{P}}\left[\lambda_{1}\left(X_{1}\right)\right]+\mathfrak{J}(\mathbb{P})\right\} \\
& =-\mu_{0}\left(\lambda_{0}^{\lambda_{1}}\right) .
\end{aligned}
$$

Therefore, the classical convex duality result (30) becomes as desired

$$
\begin{aligned}
V\left(\mu_{0}, \mu_{1}\right)=\sup _{-\lambda_{1} \in C_{b}\left(\mathbb{R}^{d}\right)}\left\{\mu_{1}\left(-\lambda_{1}\right)+\mu_{0}\left(\lambda_{0}^{\lambda_{1}}\right)\right\} & =\sup _{\lambda_{1} \in C_{b}\left(\mathbb{R}^{d}\right)}\left\{\mu_{0}\left(\lambda_{0}^{\lambda_{1}}\right)-\mu_{1}\left(\lambda_{1}\right)\right\} \\
& =\mathcal{V}\left(\mu_{0}, \mu_{1}\right) .
\end{aligned}
$$

\section{APPENDiX A}

For the appendix, let $(\Omega, \mathcal{F}, \mathbb{F}, \mathbb{P})$ be any filtered probability space. Consider a subfiltration $\mathbb{G} \subseteq \mathbb{F}$ and denote by $\mathcal{P}(\mathbb{G})$ the corresponding $\mathbb{G}$-predictable $\sigma$ field. For any process $Y$, denote by ${ }^{o} Y$ the optional projection of $Y$ with respect to $\mathbb{G}_{+}^{\mathbb{P}}$, i.e., the usual augmentation of $\mathbb{G}$. The reason why we consider the usual augmentation is that the optional projection with respect to $\mathbb{G}$ might not exist if $\mathbb{G}$ does not satisfy the usual conditions. We recall that by [31, Proposition 2.2], an $\mathbb{F}$-adapted process $X$ having càdlàg paths is an $\mathbb{F}$-semimartingale if and only if it is 
an $\mathbb{F}_{+}^{\mathbb{P}}$-semimartingale, and the characteristics associated with these filtrations are the same. If in addition $X$ is $\mathbb{G}$-adapted, the same holds true with respect to $\mathbb{G}$ (but of course, the characteristics may vary between $\mathbb{F}$ and $\mathbb{G}$ ).

We present the following useful lemma which identifies the characteristics of a semimartingale $X$ when considering a smaller filtration. The lemma is not stated in full generality, but in such a way that it fits the framework needed for this paper.

Lemma A.1. Let $(\Omega, \mathcal{F}, \mathbb{F}, \mathbb{P})$ be any filtered probability space, let $X$ be a process with càdlàg paths which is a $\mathbb{P}-\mathbb{F}$-semimartingale having absolutely continuous characteristics $\left(b_{t}^{\mathbb{F}} d t, c_{t}^{\mathbb{F}} d t, F_{t}^{\mathbb{F}} d t\right)$, and let $\mathbb{G} \subseteq \mathbb{F}$ be a subfiltration such that $X$ is $\mathbb{G}$-adapted. Moreover, assume that the canonical representation of $X$ under $\mathbb{F}$,

$$
X=X_{0}+\int_{0} b_{s}^{\mathbb{F}} d s+M^{\mathbb{F}}+\sum_{0 \leq s \leq .}\left[\Delta X_{s}-h\left(\Delta X_{s}\right)\right],
$$

satisfies $\mathbb{E}^{\mathbb{P}}\left[\int_{0}^{T}\left|b_{s}^{\mathbb{F}}\right| d s\right]<\infty$ and that the local martingale part $M^{\mathbb{F}}$ is a true $\mathbb{P}-\mathbb{F}$ martingale. Then the following hold:

(1) $X$ is a $\mathbb{P}-\mathbb{G}$-semimartingale having absolutely continuous characteristics with differential characteristics of the form

$$
\left(b^{\mathbb{G}}, c^{\mathbb{G}}, F^{\mathbb{G}}\right):=\left({ }^{o} b^{\mathbb{F}}, c^{\mathbb{F}},{ }^{o} F^{\mathbb{F}}\right),
$$

where ${ }^{o} F^{\mathbb{G}}$ is defined by setting for any $\mathcal{F} \otimes \mathcal{B}([0, T]) \otimes \mathcal{B}\left(\mathbb{R}^{d}\right)$-measurable function $W$

$$
\int_{\mathbb{R}^{d}} W(t, x)^{o} F_{t}^{\mathbb{F}}(d x):={ }^{o}\left(\int_{\mathbb{R}^{d}} W(\cdot, x) F^{\mathbb{F}}(d x)\right)_{t}, \quad t \in[0, T] .
$$

Moreover, the local martingale part $M^{\mathbb{G}}$ in the canonical representation of $X$ under $\mathbb{P}-\mathbb{G}$ is a true $\mathbb{P}-\mathbb{G}$-martingale.

(2) Furthermore, if $\mathbb{P} \times d t$-a.s., $\left(b^{\mathbb{F}}, c^{\mathbb{F}}, F^{\mathbb{F}}\right)$ take values in some $\Theta \subseteq \mathbb{R}^{d} \times$ $\mathbb{S}_{+}^{d} \times \mathcal{L}$ which is closed, convex, and satisfies Condition (B), then also $\left(b^{\mathbb{G}}, c^{\mathbb{G}}, F^{\mathbb{G}}\right) \in \Theta \mathbb{P} \times d t$-a.s.

Proof. W.l.o.g., assume that $(\Omega, \mathcal{F})=\left(\mathbb{D}\left([0, T], \mathbb{R}^{d}\right), \mathcal{B}\left(\mathbb{D}\left([0, T], \mathbb{R}^{d}\right)\right)\right)$, and let $X$ be the canonical process.

Let $\left(B^{\mathbb{F}}, C^{\mathbb{F}}, \nu^{\mathbb{F}}\right)$ be the $\mathbb{F}$-characteristics of $X$. The second characteristic can be defined as the continuous part of the finite variation process $[X]$; see e.g. [31, Proposition 6.6]. As by assumption, $X$ is $\mathbb{G}$-adapted, so is the quadratic variation process $[X]$ and hence also its continuous part. Therefore, $C^{\mathbb{G}}=C^{\mathbb{F}}$; in particular $C^{\mathbb{G}}$ is absolutely continuous $\mathbb{P}$-a.s., and $C^{\mathbb{F}}$ can be chosen to be $\mathbb{G}$-predictable. Consider the canonical representation

$$
X=X_{0}+\int_{0}^{\cdot} b_{s}^{\mathbb{F}_{+}^{\mathbb{P}}} d s+M^{\mathbb{F}_{+}^{\mathbb{P}}}+\sum_{0 \leq s \leq .}\left[\Delta X_{s}-h\left(\Delta X_{s}\right)\right]
$$

of the $\mathbb{P}-\mathbb{F}_{+}^{\mathbb{P}}$-semimartingale $X$, where $M^{\mathbb{P}_{+}^{\mathbb{P}}}$ is a true $\mathbb{P}_{-} \mathbb{F}_{+}^{\mathbb{P}}$-martingale. Therefore, by the tower property, its optional projection ${ }^{o} M^{\mathbb{F}_{+}^{\mathbb{P}}}$ (with respect to $\mathbb{G}_{+}^{\mathbb{P}}$ ) is a $\mathbb{P}-\mathbb{G}_{+}^{\mathbb{P}}$-martingale. Moreover, by the integrability condition imposed on $B^{\mathbb{F}}$, it is straightforward to verify that the process $Z:={ }^{o} B^{\mathbb{T}_{+}^{\mathbb{P}}}-\int_{0}^{\cdot o} b_{s}^{\mathbb{R}_{+}^{\mathbb{P}}} d s$ is a $\mathbb{P}_{-} \mathbb{G}_{+}^{\mathbb{P}^{-}}$martingale, too. By assumption, $X$ is $\mathbb{G}$-adapted, so $X$ is a $\mathbb{G}$ - (and hence also 
$\mathbb{G}_{+}^{\mathbb{P}}$-)semimartingale with $\mathbb{G}_{+}^{\mathbb{P}}$-canonical representation

$$
X=X_{0}+\int_{0}{ }^{o} b_{s}^{\mathbb{F}_{+}^{\mathbb{P}}} d s+\left({ }^{o} M^{\mathbb{F}_{+}^{\mathbb{P}}}+Z\right)+\sum_{0 \leq s \leq}\left[\Delta X_{s}-h\left(\Delta X_{s}\right)\right] \quad \mathbb{P} \text {-a.s. }
$$

This implies that $B^{\mathbb{G}_{+}^{\mathbb{P}}}=\int_{0}^{\cdot}{ }^{o} b_{s}^{\mathbb{F}_{+}^{\mathbb{P}}} d s$; hence by [31, Proposition 2.2], we obtain $b^{\mathbb{G}}={ }^{o} b^{\mathbb{F}}$. Moreover, $M^{\mathbb{G}}=\left({ }^{o} M^{\mathbb{F}_{+}^{\mathbb{P}}}+Z\right) \mathbb{P}$-a.s., so it is a $\mathbb{P}-\mathbb{G}$-martingale.

For the third characteristic, we have by definition that ${ }^{o} F^{\mathbb{F}}(d x) d t$ is a predictable random measure with respect to $\mathbb{G}_{+}^{\mathbb{P}}$. Moreover, we obtain for any nonnegative $\mathcal{P}\left(\mathbb{G}_{+}^{\mathbb{P}}\right) \otimes \mathcal{B}\left(\mathbb{R}^{d}\right)$-measurable function $W$ by Fubini's theorem that

$$
\mathbb{E}^{\mathbb{P}}\left[\int_{0}^{T} \int_{\mathbb{R}^{d}} W(t, x)^{o} F^{\mathbb{F}}(d x) d t\right]=\mathbb{E}^{\mathbb{P}}\left[\int_{0}^{T} \int_{\mathbb{R}^{d}} W(t, x) \mu^{X}(d x, d t)\right],
$$

which implies that the ${ }^{o} F^{\mathbb{F}}(d x) d t$ is the $\mathbb{P}_{-} \mathbb{G}_{+}^{\mathbb{P}}$-compensator of $\mu^{X}(d x, d t)$; see [21, Theorem II.1.8, p. 66]. Therefore, we conclude from [31, Proposition 2.2] that $F^{\mathbb{G}}(d x)={ }^{o} F^{\mathbb{F}}(d x)$.

Finally, for the second part, assume from now on that $\left(b^{\mathbb{F}}, c^{\mathbb{F}}, F^{\mathbb{F}}\right)$ take values in some $\Theta \subseteq \mathbb{R}^{d} \times \mathbb{S}_{+}^{d} \times \mathcal{L}$ which satisfies Condition (B) and is closed, convex. Recall the function $\bar{\varphi}$ defined in (9). By the characterization (11), we know that

$$
\begin{aligned}
& \left(b^{\mathbb{G}}, c^{\mathbb{G}}, F^{\mathbb{G}}\right) \in \Theta \mathbb{P} \times d t \text {-a.s. } \\
\Longleftrightarrow & \left(b^{\mathbb{G}}, c^{\mathbb{G}},\left(\int_{\mathbb{R}^{d}} g_{i}(x) F^{\mathbb{G}}(d x)\right)_{i \in \mathbb{N}}\right) \in \operatorname{cl}(\bar{\varphi}(\Theta)) \mathbb{P} \times d t \text {-a.s. }
\end{aligned}
$$

where $\mathcal{C}^{+}\left(\mathbb{R}^{d}\right)=\left\{g_{i} \mid i \in \mathbb{N}\right\}$; see Section 3.1. Now by assumption, we know that

$$
\left(b^{\mathbb{F}}, c^{\mathbb{F}},\left(\int_{\mathbb{R}^{d}} g_{i}(x) F^{\mathbb{F}}(d x)\right)_{i \in \mathbb{N}}\right) \in \operatorname{cl}(\bar{\varphi}(\Theta)) \mathbb{P} \times d t \text {-a.s. }
$$

Moreover, due to the first part, we have

$$
\begin{aligned}
& \left(b^{\mathbb{G}}, c^{\mathbb{G}},\left(\int_{\mathbb{R}^{d}} g_{i}(x) F^{\mathbb{G}}(d x)\right)_{i \in \mathbb{N}}\right) \\
= & \left(\mathbb{E}^{\mathbb{P}}\left[b^{\mathbb{F}} \mid \mathbb{G}_{+}^{\mathbb{P}}\right], \mathbb{E}^{\mathbb{P}}\left[c^{\mathbb{F}} \mid \mathbb{G}_{+}^{\mathbb{P}}\right],\left(\mathbb{E}^{\mathbb{P}}\left[\int_{\mathbb{R}^{d}} g_{i}(x) F^{\mathbb{F}}(d x) \mid \mathbb{G}_{+}^{\mathbb{P}}\right]\right)_{i \in \mathbb{N}}\right) \\
= & \mathbb{E}^{\mathbb{P}}\left[\left(b^{\mathbb{F}}, c^{\mathbb{F}},\left(\int_{\mathbb{R}^{d}} g_{i}(x) F^{\mathbb{F}}(d x)\right)_{i \in \mathbb{N}}\right) \mid \mathbb{G}_{+}^{\mathbb{P}}\right] .
\end{aligned}
$$

Thus, as $\operatorname{cl}(\bar{\varphi}(\Theta))$ is convex and closed,

$$
\left(b^{\mathbb{G}}, c^{\mathbb{G}},\left(\int_{\mathbb{R}^{d}} g_{i}(x) F^{\mathbb{G}}(d x)\right)_{i \in \mathbb{N}}\right) \in \operatorname{cl}(\bar{\varphi}(\Theta)) \quad \mathbb{P} \times d t \text {-a.s. }
$$

Remark A.2. Observe that in the setting of Lemma A.1 we have for any $\delta>0$ that

$$
\mathbb{E}^{\mathbb{P}}\left[\int_{0}^{T} \int_{\{|x| \leq \delta\}}|x|^{2} F_{t}^{\mathbb{F}}(d x) d t\right]=\mathbb{E}^{\mathbb{P}}\left[\int_{0}^{T} \int_{\{|x| \leq \delta\}}|x|^{2} F_{t}^{\mathbb{G}}(d x) d t\right] .
$$

\section{ACKNOWLEDGment}

The authors would like to thank the referees for their helpful and constructive comments that greatly contributed to improving the paper. 


\section{REFERENCES}

[1] Mathias Beiglböck, Pierre Henry-Labordère, and Friedrich Penkner, Model-independent bounds for option prices - a mass transport approach, Finance Stoch. 17 (2013), no. 3, 477501, DOI 10.1007/s00780-013-0205-8. MR3066985

[2] Mathias Beiglböck, Pierre Henry-Labordère, and Nizar Touzi, Monotone martingale transport plans and Skorokhod embedding, Stochastic Process. Appl. 127 (2017), no. 9, 3005-3013, DOI 10.1016/j.spa.2017.01.004. MR.3682121

[3] Mathias Beiglböck and Nicolas Juillet, On a problem of optimal transport under marginal martingale constraints, Ann. Probab. 44 (2016), no. 1, 42-106, DOI 10.1214/14-AOP966. MR3456332

[4] Mathias Beiglböck, Marcel Nutz, and Nizar Touzi, Complete duality for martingale optimal transport on the line, Ann. Probab. 45 (2017), no. 5, 3038-3074, DOI 10.1214/16-AOP1131. MR.3706738

[5] Dimitri P. Bertsekas and Steven E. Shreve, Stochastic optimal control, The discrete time case, Mathematics in Science and Engineering, vol. 139, Academic Press, Inc. [Harcourt Brace Jovanovich, Publishers], New York-London, 1978. MR511544

[6] Patrick Billingsley, Convergence of probability measures, 2nd ed., Wiley Series in Probability and Statistics: Probability and Statistics, John Wiley \& Sons, Inc., New York, 1999. MR 1700749

[7] V. I. Bogachev, Measure theory. Vol. I, II, Springer-Verlag, Berlin, 2007. MR2267655

[8] Luciano Campi, Ismail Laachir, and Claude Martini, Change of numeraire in the twomarginals martingale transport problem, Finance Stoch. 21 (2017), no. 2, 471-486, DOI 10.1007/s00780-016-0322-2. MR 3626622

[9] A. B. Cruzeiro and R. Lassalle, Weak calculus of variations for functionals of laws of semimartingales, preprint, arXiv: 1501.05134, 2015.

[10] Jean-Dominique Deuschel and Daniel W. Stroock, Large deviations, Pure and Applied Mathematics, vol. 137, Academic Press, Inc., Boston, MA, 1989. MR997938

[11] Yan Dolinsky and H. Mete Soner, Martingale optimal transport and robust hedging in continuous time, Probab. Theory Related Fields 160 (2014), no. 1-2, 391-427, DOI 10.1007/s00440013-0531-y. MR3256817

[12] Yan Dolinsky and H. Mete Soner, Martingale optimal transport in the Skorokhod space, Stochastic Process. Appl. 125 (2015), no. 10, 3893-3931, DOI 10.1016/j.spa.2015.05.009. MR.3373308

[13] Arash Fahim and Yu-Jui Huang, Model-independent superhedging under portfolio constraints, Finance Stoch. 20 (2016), no. 1, 51-81, DOI 10.1007/s00780-015-0284-9. MR3441286

[14] A. Galichon, P. Henry-Labordère, and N. Touzi, A stochastic control approach to no-arbitrage bounds given marginals, with an application to lookback options, Ann. Appl. Probab. 24 (2014), no. 1, 312-336, DOI 10.1214/13-AAP925. MR3161649

[15] Gaoyue Guo, Xiaolu Tan, and Nizar Touzi, Tightness and duality of martingale transport on the Skorokhod space, Stochastic Process. Appl. 127 (2017), no. 3, 927-956, DOI 10.1016/j.spa.2016.07.005. MR3605716

[16] Pierre Henry-Labordère, Jan Obłój, Peter Spoida, and Nizar Touzi, The maximum maximum of a martingale with given $n$ marginals, Ann. Appl. Probab. 26 (2016), no. 1, 1-44, DOI 10.1214/14-AAP1084. MR3449312

[17] Pierre Henry-Labordère and Nizar Touzi, An explicit martingale version of the onedimensional Brenier theorem, Finance Stoch. 20 (2016), no. 3, 635-668, DOI 10.1007/s00780016-0299-x. MR3519164

[18] D. Hobson, Robust hedging of the lookback option, Finance Stoch. 2(1998), no. 4, 329-347.

[19] David Hobson, The Skorokhod embedding problem and model-independent bounds for option prices, Paris-Princeton Lectures on Mathematical Finance 2010, Lecture Notes in Math., vol. 2003, Springer, Berlin, 2011, pp. 267-318, DOI 10.1007/978-3-642-14660-2_4. MR2762363

[20] J. Jacod, J. Mémin, and M. Métivier, On tightness and stopping times, Stochastic Process. Appl. 14 (1983), no. 2, 109-146, DOI 10.1016/0304-4149(83)90067-4. MR679668

[21] Jean Jacod and Albert N. Shiryaev, Limit theorems for stochastic processes, 2nd ed., Grundlehren der Mathematischen Wissenschaften [Fundamental Principles of Mathematical Sciences], vol. 288, Springer-Verlag, Berlin, 2003. MR1943877 
[22] A. Jakubowski, The almost sure Skorokhod representation for subsequences in nonmetric spaces (English, with Russian summary), Teor. Veroyatnost. i Primenen. 42 (1997), no. 1, 209-216, DOI 10.1137/S0040585X97976052; English transl., Theory Probab. Appl. 42 (1997), no. 1, 167-174 (1998). MR 1453342

[23] Adam Jakubowski, A non-Skorohod topology on the Skorohod space, Electron. J. Probab. 2 (1997), no. 4, 21 pp., DOI 10.1214/EJP.v2-18. MR.1475862

[24] L. Kantorovitch, On the translocation of masses, C. R. (Doklady) Acad. Sci. URSS (N.S.) 37 (1942), 199-201. MR.0009619

[25] Hans G. Kellerer, Duality theorems for marginal problems, Z. Wahrsch. Verw. Gebiete 67 (1984), no. 4, 399-432, DOI 10.1007/BF00532047. MR761565

[26] J.-P. Lepeltier and B. Marchal, Problème des martingales et équations différentielles stochastiques associées à un opérateur intégro-différentiel (French), Ann. Inst. H. Poincaré Sect. B (N.S.) 12 (1976), no. 1, 43-103. MR0413288

[27] P.-A. Meyer and W. A. Zheng, Tightness criteria for laws of semimartingales (English, with French summary), Ann. Inst. H. Poincaré Probab. Statist. 20 (1984), no. 4, 353-372. MR771895

[28] Toshio Mikami, Optimal control for absolutely continuous stochastic processes and the mass transportation problem, Electron. Comm. Probab. 7 (2002), 199-213, DOI 10.1214/ECP.v71061. MR1937905

[29] Toshio Mikami, Two end points marginal problem by stochastic optimal transportation, SIAM J. Control Optim. 53 (2015), no. 4, 2449-2461, DOI 10.1137/14099070X. MR.3383307

[30] Toshio Mikami and Michèle Thieullen, Duality theorem for the stochastic optimal control problem, Stochastic Process. Appl. 116 (2006), no. 12, 1815-1835, DOI 10.1016/j.spa.2006.04.014. MR2307060

[31] Ariel Neufeld and Marcel Nutz, Measurability of semimartingale characteristics with respect to the probability law, Stochastic Process. Appl. 124 (2014), no. 11, 3819-3845, DOI 10.1016/j.spa.2014.07.006. MR3249357

[32] Ariel Neufeld and Marcel Nutz, Nonlinear Lévy processes and their characteristics, Trans. Amer. Math. Soc. 369 (2017), no. 1, 69-95, DOI 10.1090/tran/6656. MR3557768

[33] Ariel Neufeld and Marcel Nutz, Robust utility maximization with Lévy processes, Math. Finance 28 (2018), no. 1, 82-105, DOI 10.1111/mafi.12139. MR3758917

[34] Jan Obłój, The Skorokhod embedding problem and its offspring, Probab. Surv. 1 (2004), 321-390, DOI 10.1214/154957804100000060. MR2068476

[35] R. Rebolledo, La méthode des martingales appliquée à l'étude de la convergence en loi de processus (French), Bull. Soc. Math. France Mém. 62 (1979), v+125 pp. (1980). MR568153

[36] A. V. Skorohod, Limit theorems for stochastic processes (Russian, with English summary), Teor. Veroyatnost. i Primenen. 1 (1956), 289-319. MR0084897

[37] F. Stebegg, Model-independent pricing of asian options via optimal martingale transport, preprint, arXiv:1412.1429, 2014.

[38] Daniel W. Stroock and S. R. Srinivasa Varadhan, Multidimensional diffusion processes, Grundlehren der Mathematischen Wissenschaften [Fundamental Principles of Mathematical Sciences], vol. 233, Springer-Verlag, Berlin-New York, 1979. MR532498

[39] Xiaolu Tan and Nizar Touzi, Optimal transportation under controlled stochastic dynamics, Ann. Probab. 41 (2013), no. 5, 3201-3240, DOI 10.1214/12-AOP797. MR3127880

[40] Cédric Villani, Optimal transport, Old and new, Grundlehren der Mathematischen Wissenschaften [Fundamental Principles of Mathematical Sciences], vol. 338, Springer-Verlag, Berlin, 2009. MR2459454

[41] W. A. Zheng, Tightness results for laws of diffusion processes application to stochastic mechanics (English, with French summary), Ann. Inst. H. Poincaré Probab. Statist. 21 (1985), no. 2, 103-124. MR798890

Department of Mathematics, ETH Zurich, 8092 Zurich, Switzerland

Email address: chong.liu@math.ethz.ch

Division of Mathematical Sciences, Nanyang Technological University, Singapore

Email address: ariel.neufeld@ntu.edu.sg 